\title{
Phylogeny and character evolution in the Dacrymycetes, and systematics of Unilacrymaceae and Dacryonaemataceae fam. nov.
}

\author{
J.C. Zamora ${ }^{1,2}$, S. Ekman ${ }^{1}$
}

\author{
Key words \\ Carotenoids \\ coalescence analyses \\ cytology \\ Dacrymycetes \\ Dacryonaema \\ species delimitations \\ Unilacryma
}

\begin{abstract}
We present a multilocus phylogeny of the class Dacrymycetes, based on data from the 18S, ITS, 28S, $R P B 1, R P B 2, T E F-1 \alpha, 12 S$, and ATP6 DNA regions, with c. 90 species including the types of most currently accepted genera. A variety of methodological approaches was used to infer phylogenetic relationships among the Dacrymycetes, from a supermatrix strategy using maximum likelihood and Bayesian inference on a concatenated dataset, to coalescence-based calculations, such as quartet-based summary methods of independent single-locus trees, and Bayesian integration of single-locus trees into a species tree under the multispecies coalescent. We evaluate for the first time the taxonomic usefulness of some cytological phenotypic characters, i.e., vacuolar contents (vacuolar bodies and lipid bodies), number of nuclei of recently discharged basidiospores, and pigments, with especial emphasis on carotenoids. These characters, along with several others traditionally used for the taxonomy of this group (basidium shape, presence and morphology of clamp connections, morphology of the terminal cells of cortical/ marginal hyphae, presence and degree of ramification of the hyphidia), are mapped on the resulting phylogenies and their evolution through the class Dacrymycetes discussed. Our analyses reveal five lineages that putatively represent five different families, four of which are accepted and named. Three out of these four lineages correspond to previously circumscribed and published families (Cerinomycetaceae, Dacrymycetaceae, and Unilacrymaceae), and one is proposed as the new family Dacryonaemataceae. Provisionally, only a single order, Dacrymycetales, is accepted within the class. Furthermore, the systematics of the two smallest families, Dacryonaemataceae and Unilacrymaceae, are investigated to the species level, using coalescence-based species delimitation on multilocus DNA data, and a detailed morphological study including morphometric analyses of the basidiospores. Three species are accepted in Dacryonaema, the type, Da. rufum, the newly combined Da. macnabbii (basionym Dacrymyces macnabbii), and a new species named Da. macrosporum. Two species are accepted in Unilacryma, the new U. bispora, and the type, $U$. unispora, the latter treated in a broad sense pending improved sampling across the Holarctic.
\end{abstract}

Article info Received: 22 August 2019; Accepted: 24 October 2019; Published: 24 April 2020.

\section{INTRODUCTION}

The Dacrymycetes (Basidiomycota) is one of the three widely accepted classes in the Agaricomycotina, sister to the Agaricomycetes. It is a monophyletic group comprised of widespread, saprotrophic fungi, mainly characterised by the strikingly long bisterigmate (forked) holobasidia (Hibbett et al. 2007), Unilacryma unispora being the only known exception (Shirouzu et al. 2013). The class is further characterised at the ultrastructural level by the presence of continuous parenthosomes covering the dolipore-type septa (Oberwinkler 1994, Wells 1994). In addition, basidiomata are gelatinous to waxy or fleshy-cartilaginous, displaying a wide variation in size and shape, and very frequently yellowish to orange due to the presence of carotenoids (Gill \& Steglich 1987), the main pigments of this group (Fig. 1).

The Dacrymycetes are typically brown-rotters (Worrall et al. 1997), although some have been reported to degrade considerable amounts of lignin (Seifert 1983). Gene contents from two Calocera genomes also suggests a brown-rotting lifestyle (Nagy et al. 2015), and Dacrymycetes are the clade with the oldest stem age among Basidiomycota wood decomposers. As a result, their ancestors were likely among the first basidio-

\footnotetext{
Museum of Evolution, Uppsala University, Norbyvägen 16, SE-75236 Uppsala, Sweden; corresponding author e-mail: jczamora@em.uu.se.

2 Departamento de Biología Vegetal II, Facultad de Farmacia, Universidad Complutense de Madrid, Ciudad Universitaria, plaza de Ramón y Cajal s/n, E-28040, Madrid, Spain.
}

mycetous fungi able to decay wood (Oberwinkler 1994, Kirk et al. 2008, Floudas et al. 2012). Floudas et al. (2012), based on genome-scale data (26 DNA regions) in which Dacrymycetes was represented by a single species, estimated the stem age of the class to around 350 million years ago (Ma). A similar result, based on phylogenetic analysis of up to six DNA regions, was reached by Zhao et al. (2017), who reported a mean stem age of $358 \mathrm{Ma}$. Garnica et al. (2016) estimated the stem age to around $300 \mathrm{Ma}$ using four DNA regions, but the Dacrymycetes clade was represented by only two species in the Dacrymycetaceae. Recently, Varga et al. (2019) performed a variety of dating analyses on a phylogenomic dataset including three Dacrymycetaceae species and obtained mean stem ages for the Dacrymycetes around 278-504 Ma, although mostly in the 360-385 Ma range. In view of this, the ancestors of this class and the Agaricomycetes seem to have split during the Upper Devonian or the Carboniferous.

The Dacrymycetes was traditionally comprised of a single order, Dacrymycetales, with two families, Dacrymycetaceae and Cerinomycetaceae, but a second order called Unilacrymales was erected to accommodate the single known species with simple basidia, Unilacryma unispora (Shirouzu et al. 2013). The two orders were accepted in a recent study dealing with high-level taxonomy of the Basidiomycota (Zhao et al. 2017), although no species of Cerinomycetaceae were included.

Generic boundaries within the Dacrymycetes have largely remained unaltered since Martin (1952), except for Dicellomyces having been transferred to the Ustilaginomycotina (Kirk et al. 

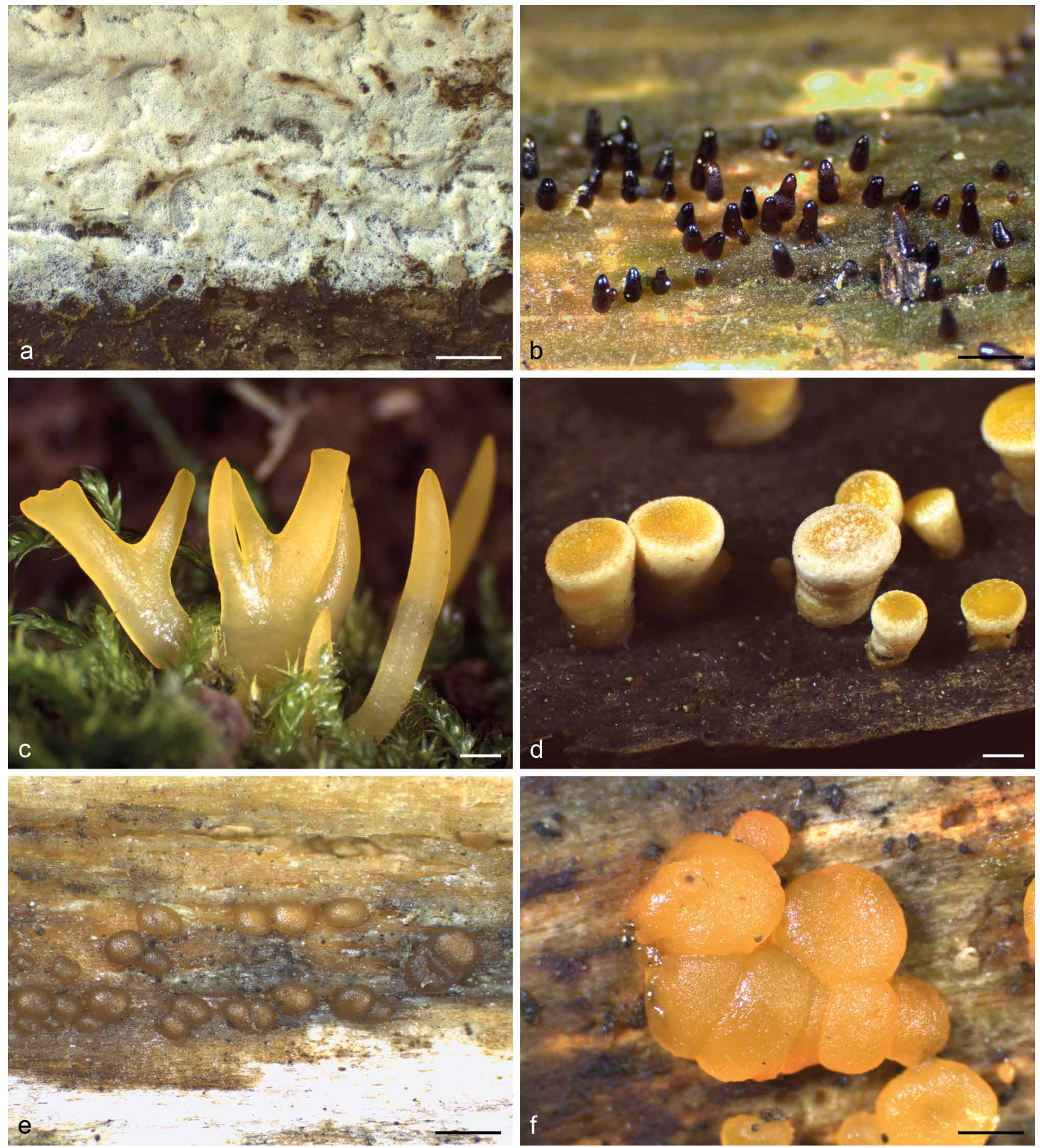

Fig. 1 Morphological variation of the fruitbodies in the Dacrymycetes. a. Resupinate and almost unpigmented in Cerinomyces sp. (O 247959); b. sterile dark conical stalks in Dacryonaema rufum (UPS F-941005); c. clavarioid and yellow in Calocera cornea (UPS F-940775); d. turbinate, stalked and with a yelloworange hymenium in Ditiola radicata (UPS F-939961); e. pulvinate, sessile and brownish in Unilacryma bispora (UPS F-941266); f. pulvinate, sessile and bright orange in Dacrymyces ovisporus (UPS F-940139). All in fresh conditions except a. - Scale bars $=1 \mathrm{~mm}$.

2008). The shape of the fruitbodies, basidioma growth direction, presence or absence of a stipe, wall thickness of internal hyphae, and cell differentiation of cortical/marginal hyphae (partially related to the presence/absence of a sterile cortex) are among the most important taxonomic characters traditionally used to distinguish the genera (Fig. 1-2). In a revision based mainly on these characters, Oberwinkler (2014) accepted the following 13 genera: Arrhytidia, Calocera, Cerinomyces, Dacrymyces, Dacryomitra, Dacryonaema, Dacryopinax, Dacryoscyphus, Ditiola, Femsjonia, Guepiniopsis, Heterotextus, and Unilacryma. However, some of the mentioned characters, e.g., the shape of the fruitbodies, were demonstrated to be homo- plastic by Shirouzu et al. (2013), and several common genera are polyphyletic (e.g., Calocera, Cerinomyces, Dacrymyces). In the same study, it was shown that other phenotypic traits, such as the presence or absence of clamp connections, not previously used to distinguish genera, are much better correlated with the phylogeny based on DNA data. For instance, species with and without clamp connections in Calocera, or in Cerinomyces, are not closely related.

Carotenoid pigments typical of the Dacrymycetes are synthetised by other fungal groups, but they are relatively unusual among non-photosynthetic eukaryotes (Echavarri-Erasun \& Johnson 2002). They are prominent only in a few fungal taxa, 


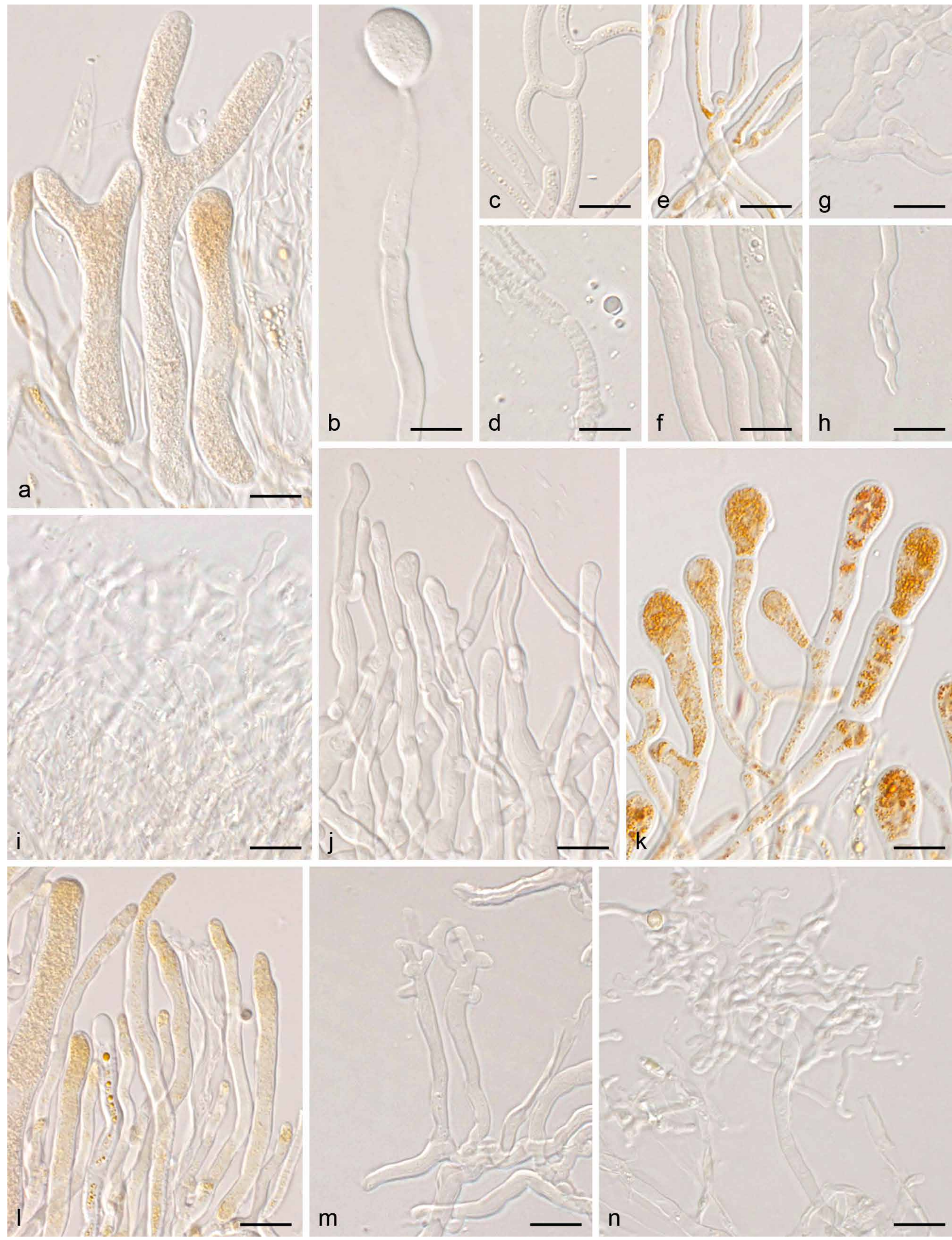

Fig. 2 Micromorphology of traditionally used taxonomic characters. a. Bisterigmate basidia in Dacrymyces estonicus (UPS F-941253); b. unisterigmate basidium in Unilacryma unispora (UPS F-941283); c-d. clampless septa in D. stillatus (c, UPS F-939816) and Dacryonaema macrosporum (d, holotype); e-f. short and rounded clamp connections in D. chrysocomus s.lat. 2 (e, UPS F-940946) and U. bispora (f, UPS F-941259); g-h. long and comparatively angulose clamp connections in Da. macnabbii (g, UPS F-940954) and Da. rufum (h, UPS F-941005); i-k. terminal cells of cortical/marginal hyphae, branched and gelatinised in Da. macnabbii (i, UPS F-940966), simple and not swollen in D. tortus s.lat. 4 (j, UPS F-941252), and clearly dilated in D. estonicus (k, UPS F-941253); I-n. hyphidia, simple in D. ovisporus (I, UPS F-940139), with short apical branches in D. tortus s.lat. 3 (m, UPS F-941018), and heavily branched in Da. macnabbii (n, UPS F-940950). - Scale bars $=10 \mu \mathrm{m}$. 
and as a result they have been used as taxonomic characters both in Ascomycota (e.g., Eckblad 1968, Schumacher 1990, Baral 2004) and Basidiomycota (e.g., Arpin \& Fiasson 1971, Olariaga et al. 2015). Their acquisition and secondary loss in different phylogenetic lineages are well-known (Hansen et al. 2013, Olariaga et al. 2015), but remains to be investigated in the Dacrymycetes.

Species delimitations are challenging in the Dacrymycetes. According to Hibbett et al. (2007), this is the smallest class of the subphylum Agaricomycotina, with 110-120 species known so far (Kirk et al. 2008, Shirouzu et al. 2009, 2017). However, judging from recent studies and our experience with fresh and herbarium samples, the real number of species is certainly larger, probably over 200. Basidiospore dimensions and septation, and the morphology of hyphidia are important characters for morphological species delimitation (McNabb 1964, 1965a-e, 1966, 1973), but their variation may be considerable even within a single fruitbody. Shirouzu et al. (2017) provided the most complete phylogeny in terms of included vouchers with both DNA data and morphological data from fruitbodies. Some of the tentatively identified species turned out to be polyphyletic (e.g., Calocera cf. guepinioides, Cerinomyces crustulinus, Ce. pallidus, Dacrymyces microsporus, $D$. minor, D. novae-zelandiae, $D$. stillatus) or appeared on exceptionally long branches within their clade, indicating there is considerable sequence variation (e.g., Calocera cornea, Ca. lutea, Dacrymyces capitatus, Heterotextus miltinus). These groups, among others, may contain so far unrecognised species.

The class was subject to a worldwide monograph by Robert McNabb, who described new taxa from a variety of countries and ecozones in a series of studies (McNabb 1964, 1965a-e, $1966,1973)$ based on numerous other previous treatments, including those of, e.g., Brasfield (1938) and Kennedy (1959a, b) from the USA, or Kobayasi (1939a, b) from East Asia. During the last decade, significant contributions to the understanding of this group, including several studies based on morphological, molecular and ecological data, mainly on the Japanese and Australasian species, have been published by, e.g., Shirouzu et al. (2009, 2013, 2016, 2017). In Europe, this group sparked the interest of Neuhoff (1936), and was later addressed in considerable detail by Donk (1966) and Raitviir (1967, including a substantial part of northern Asia). Reid (1974) presented a monograph focused on the British taxa, including 20 species, two of which were proposed as new. Since then, however, there have been no thorough revision of the European taxa with modern techniques. In the Nordic countries, Nannfeldt's (1947) monotypic genus Dacryonaema stands out as infrequently recorded, mainly from Northern Europe (Torkelsen 1997, http:// www.gbif.org/species/2512811), although it has also been reported from North America (Brough \& Bandoni 1975, Redhead 1997). No DNA data were available for Dacryonaema at the beginning of this study, and the phylogenetic relationships of this morphologically deviant genus with the remaining species of Dacrymycetes are largely unknown (Oberwinkler 2014). On the other hand, the monotypic genus Unilacryma, described from North America and recorded from Japan, has recently been found also in Europe (Dam \& Dam 2014). Shirouzu et al. (2013) commented on the morphological characteristics of the only known species, $U$. unispora, and compared it with another morphologically close species, Dacrymyces ovisporus, based on basidia and basidiospore characters. Dacrymyces ovisporus was described from Germany and has a wide distribution, including, e.g., northern Europe (Torkelsen 1997) as well as North America (Bandoni 1963). No DNA sequence data of this species are available yet, and its taxonomic position remains unclear.
The objectives of this paper are:

(i) to establish phylogenetic relationships among genera and species in the Dacrymycetes (including the genus Dacryonaema) based on multilocus DNA data;

(ii) to identify phenotypic characters that allow the recognition of the phylogenetic lineages at family level, paying particular attention to those previously overlooked; and

(iii) to revise the systematics and nomenclature of Dacryonaemataceae and Unilacrymaceae based on an integrative taxonomy approach, with special emphasis on the European taxa.

The third aim was taxonomically and geographically restricted because a wider scope would have been hampered by the extensive diversity and the large number of published names. The systematics of the larger Cerinomycetaceae and Dacrymycetaceae will be investigated in collaboration with other researchers in forthcoming studies.

\section{MATERIAL AND METHODS}

\section{Taxon and marker sampling}

We sampled representatives for an overall phylogeny based on their morphology and phylogenetic position in previous studies. Due to the similarity of names, Calocera is abbreviated as 'Ca.', Cerinomyces as 'Ce.', Dacrymyces as 'D.', Dacryonaema as 'Da.', Ditiola as 'Di.', whereas Dacryomitra, Dacryopinax and Dacryoscyphus are not abbreviated. We generated new data from five species in the Cerinomycetaceae, viz., Cerinomyces $\mathrm{sp}$. (resupinate morphology and \pm dry fruitbodies) and $D$. tortus s.lat. (four species with 'dacrymycetoid' and 'coalescing' morphologies, with \pm gelatinous fruitbodies); the following species in the Dacrymycetaceae, viz., Ca. cornea, Ca. viscosa, $D$. chrysocomus s.lat., $D$. aff. chrysospermus, D. estonicus, D. cf. minutus, D. pinacearum, D. stillatus, Dacryomitra pusilla, Dacryopinax elegans, Ditiola radicata, Femsjonia peziziformis s.lat., Guepiniopsis buccina, and Heterotextus miltinus s.lat.; Unilacryma unispora s.lat. in the Unilacrymaceae, and Dacryonaema rufum with an unknown placement. Additionally, we included Dacrymyces macnabbii and $D$. ovisporus, which have not been included in any phylogeny yet, and two unidentified species that, according to morphological and preliminary molecular data, may belong in Dacryonaema or Unilacryma. We paid particular attention to selecting only visibly clean and non-parasitised fruitbodies. In addition, we added all available Dacrymycetes samples in GenBank being represented by at least three of the eight DNA regions selected (see below), for this purpose considering the ITS (ITS1 + 5.8S + ITS2) as a single region (Table 1 ). This includes mostly samples from the AFTOL project (Hibbett et al. 2007), the studies on the Dacrymycetes by Shirouzu et al. (2007, 2009, 2013, 2016), and four Dacrymycetes species in MycoCosm (Grigoriev et al. 2014). In order to better assess the consistency of some phenotypic characters at the family level, we expanded the taxonomic sampling to include most available species with both a reasonable amount of molecular and morphological data. For samples with at least two DNA regions available in GenBank, we included data of up to two individuals of each taxon identified to species level when it was either not previously included or represented by a single individuum. When selecting among multiple available sequences, preference was given first to type specimens and second to the longest sequences; most of these additional sequences were generated by Shirouzu et al. (2017). Finally, and as an exception, a partial 28 S sequence of Dacryoscyphus chrysochilus (AY604567, from the holotype specimen) was included because this was the only available DNA sequence of the type of Dacryoscyphus. 

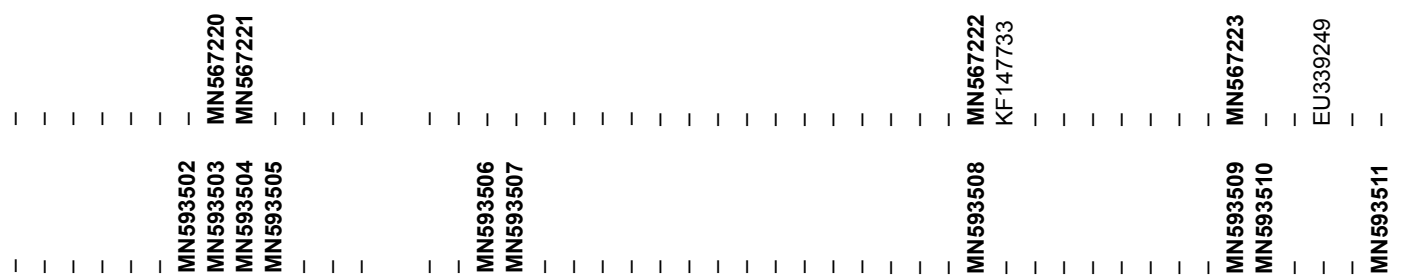

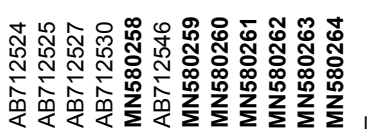

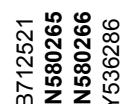

焉
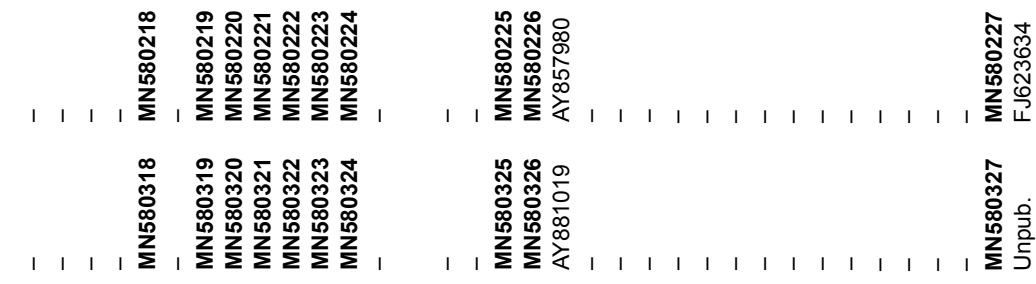

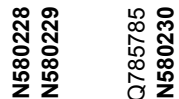

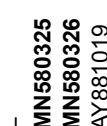

商.

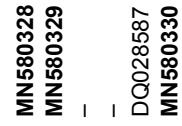

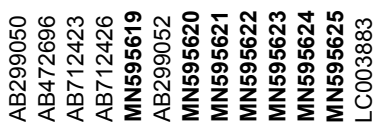

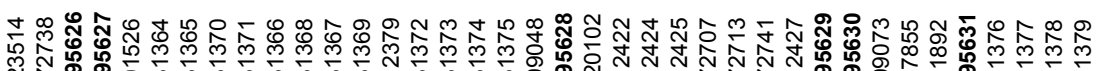

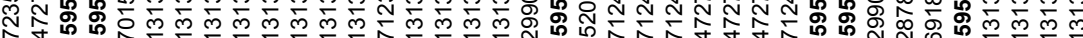

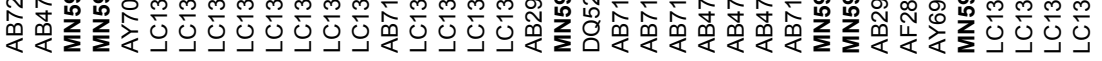

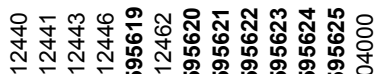

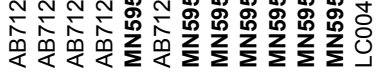

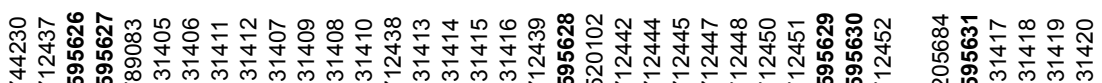

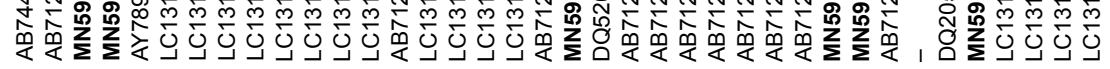

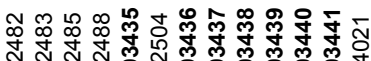

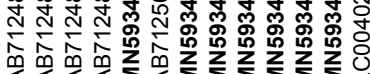

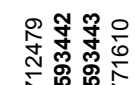

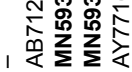

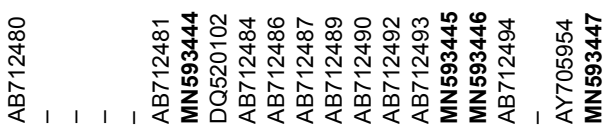

일

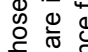

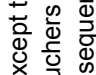
要要这

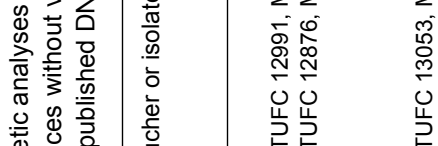

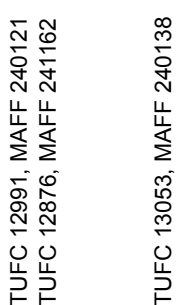

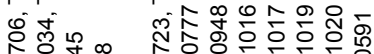

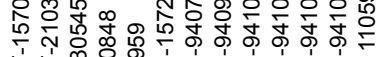

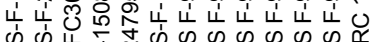

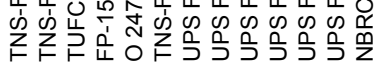

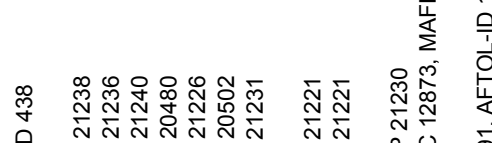

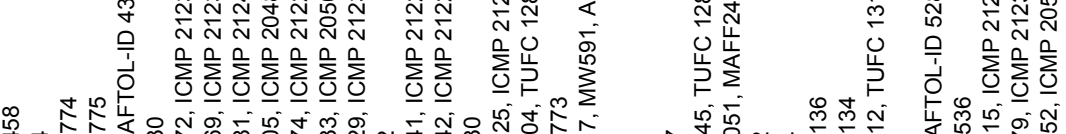

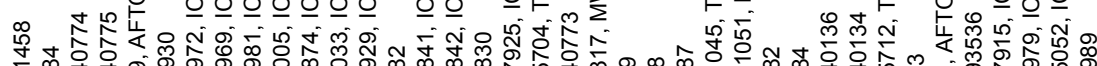

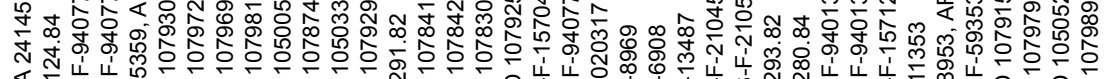

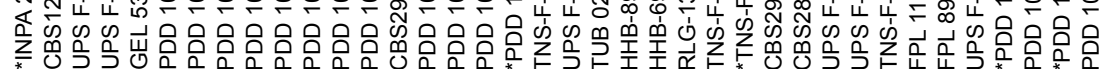

mInninn

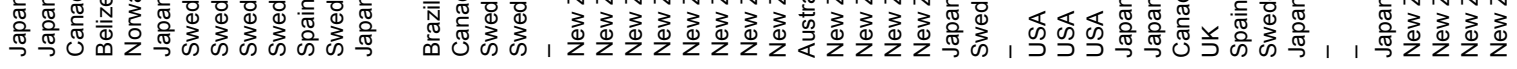

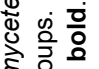

츤은 $\cong$

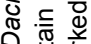

它

员 ธ。

हे 올 흘

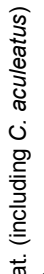

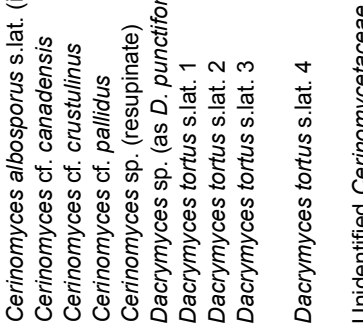

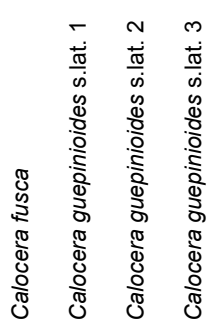

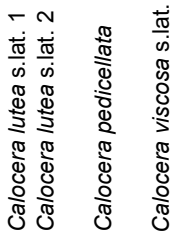
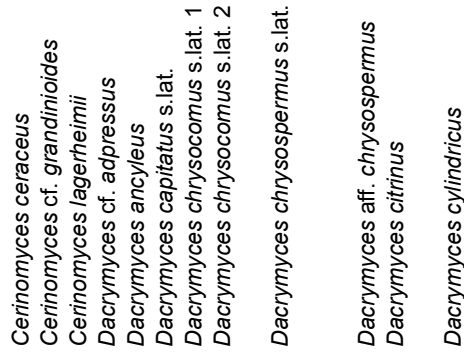


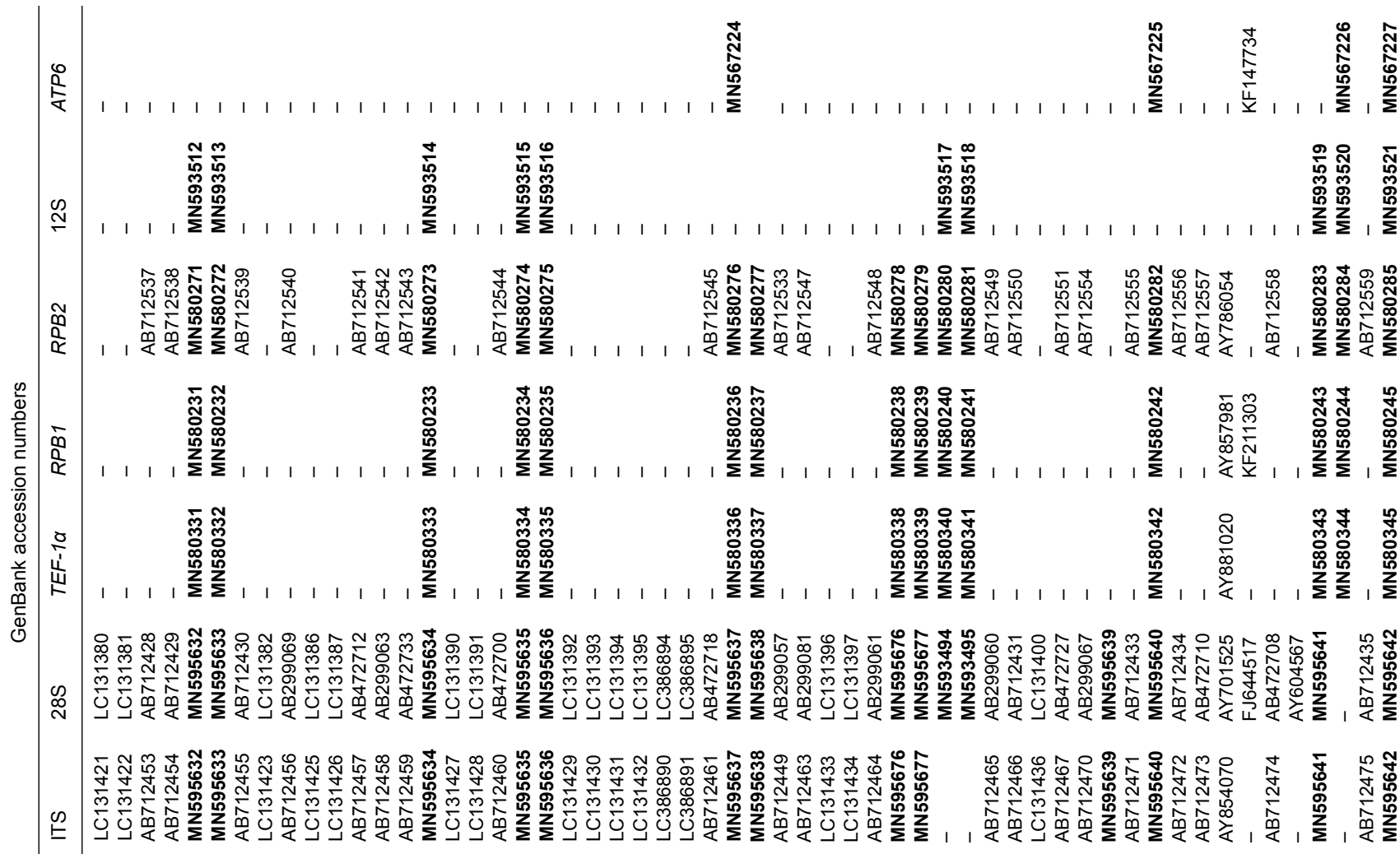

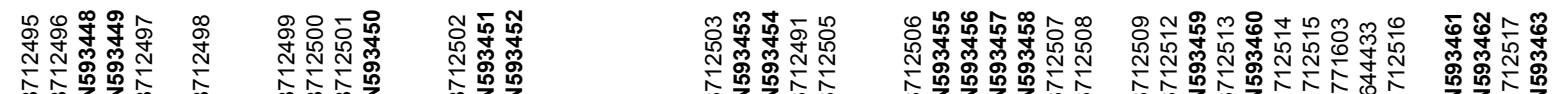

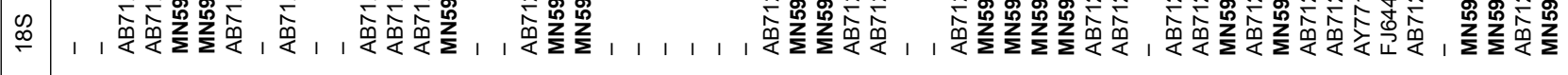

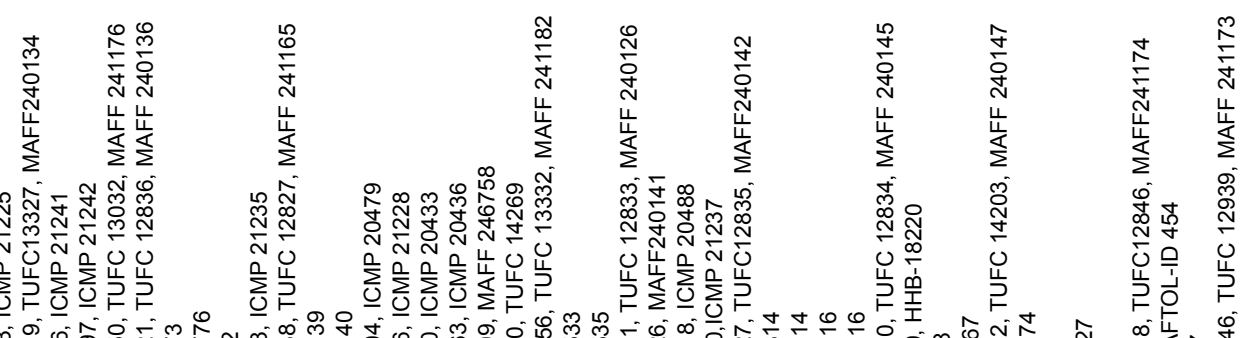

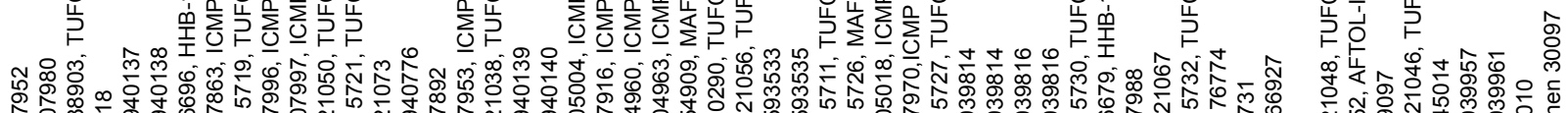

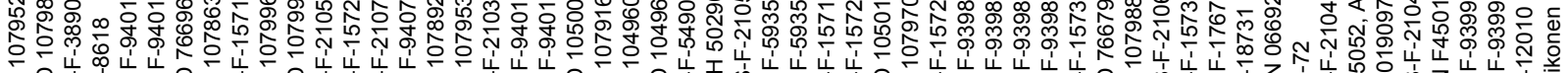

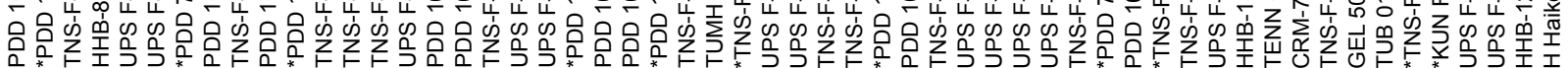

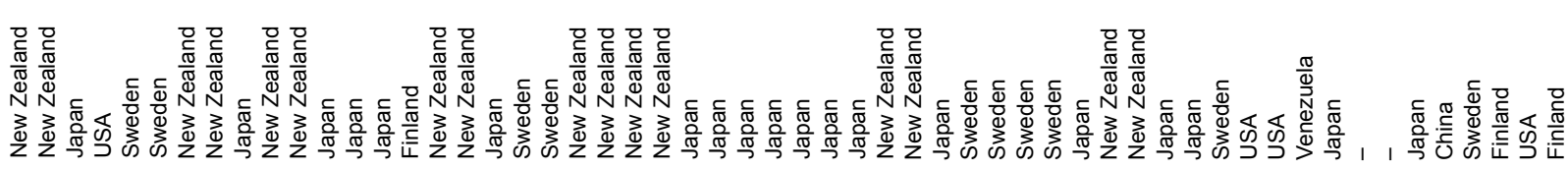

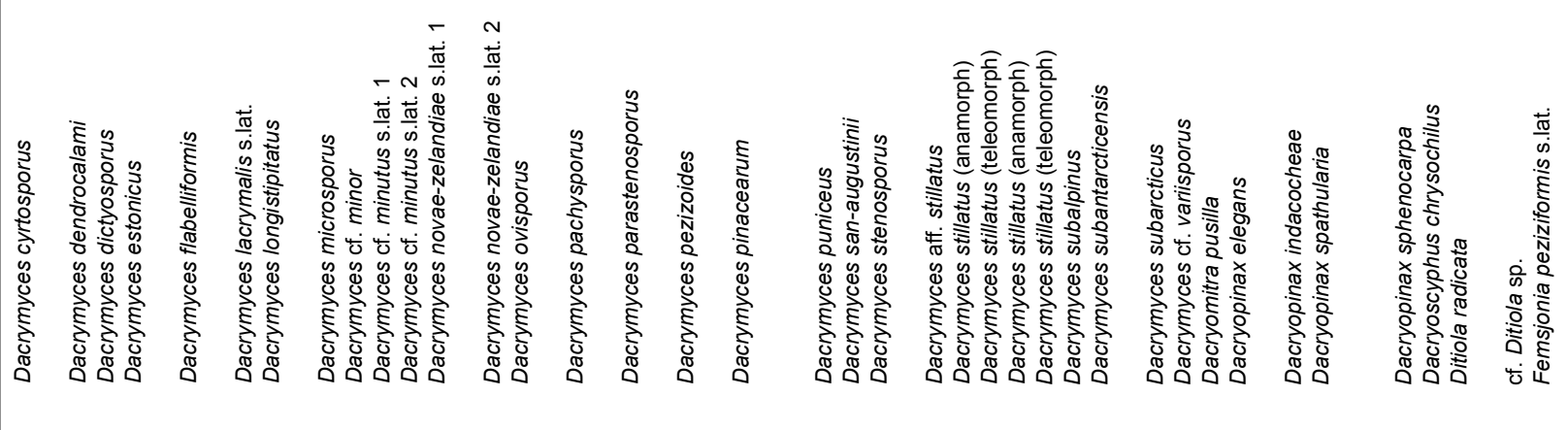




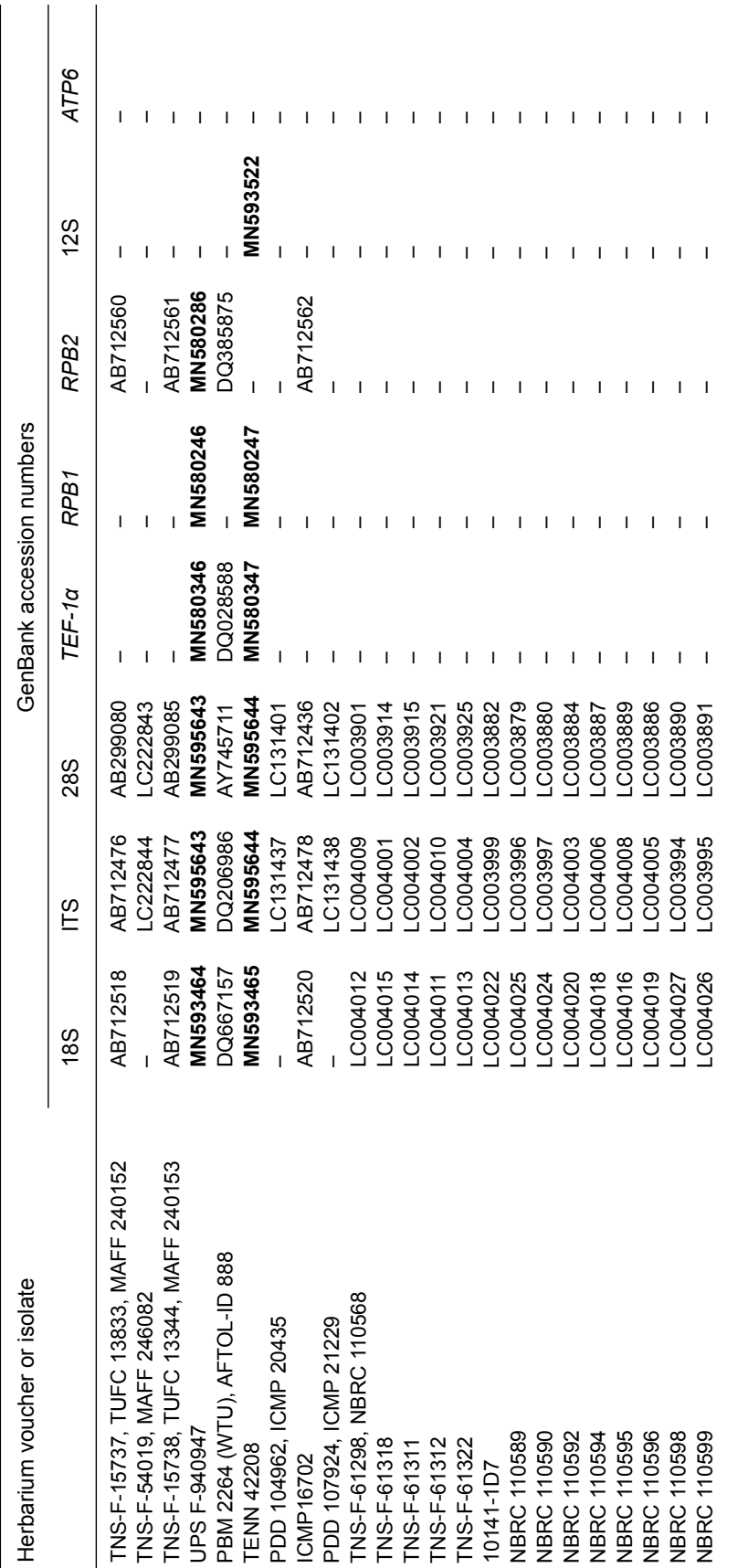

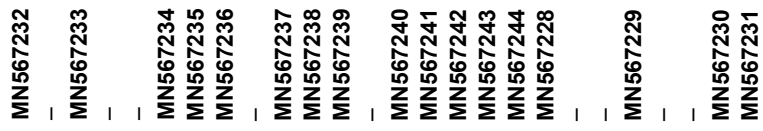

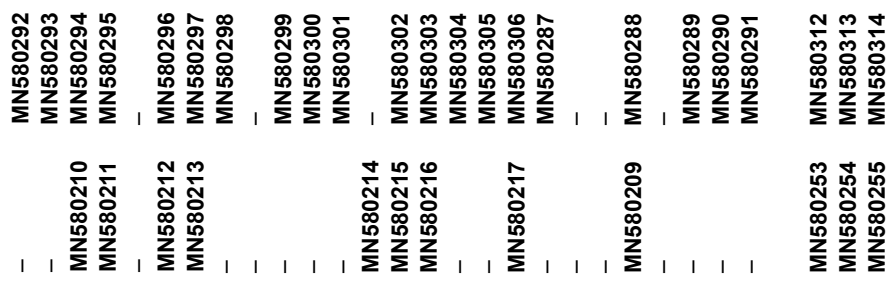

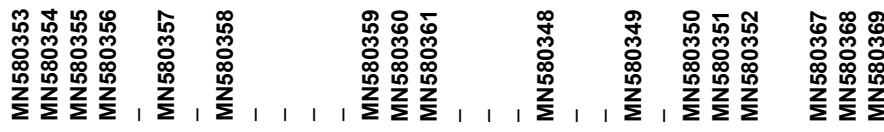

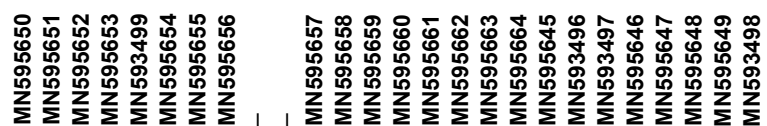

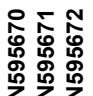

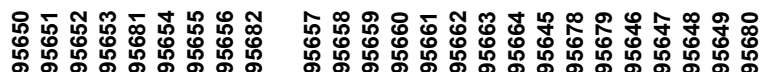

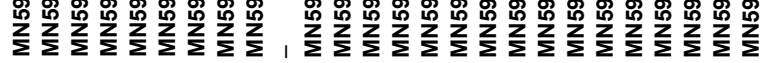

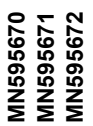

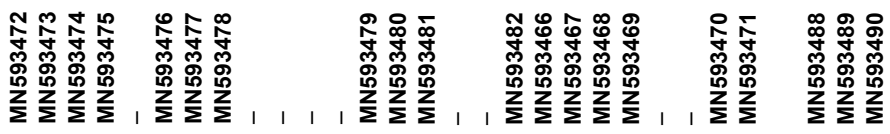

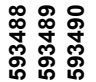

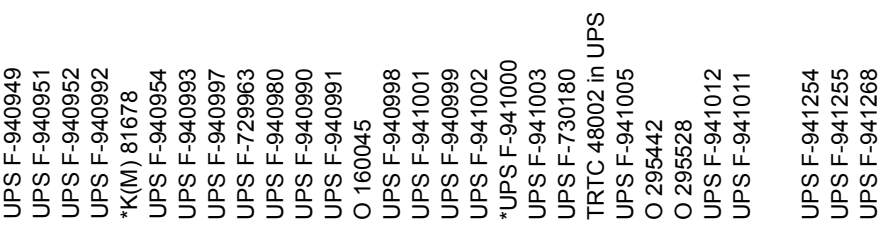

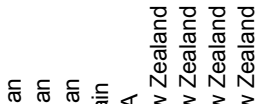

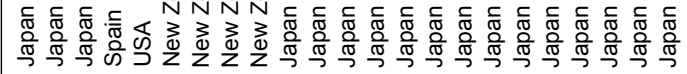

MInImmmmmm 


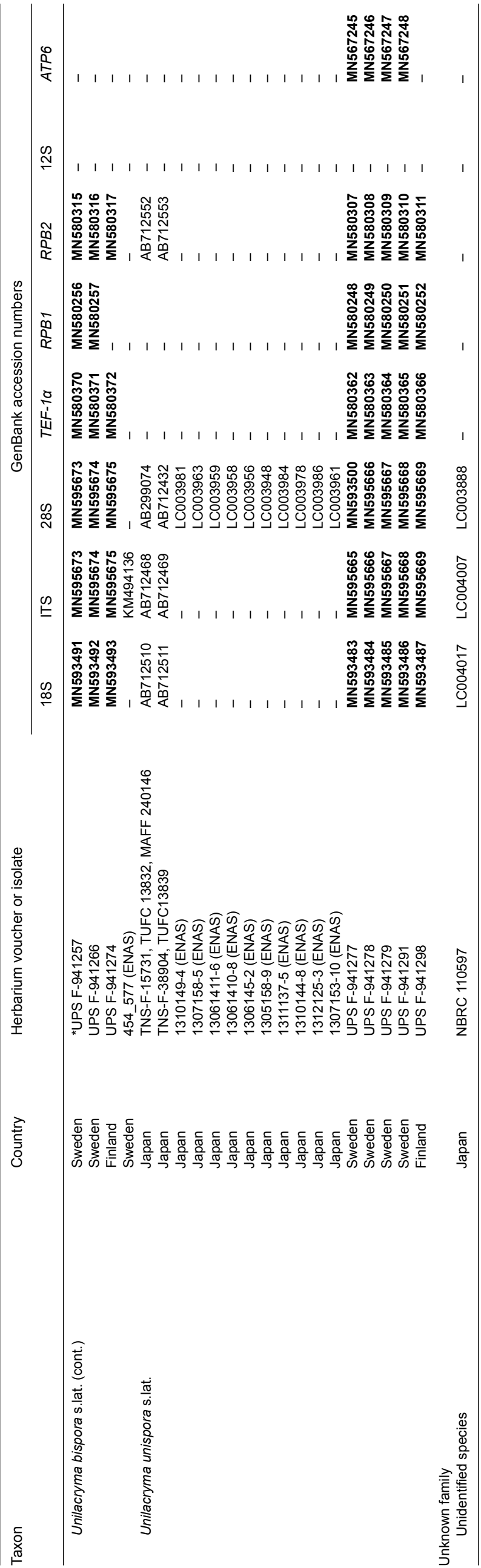

This sampling comprised c. 90 ingroup species (not all of them identified) and included, at least in a broad sense, the types of all genera accepted by McNabb (1964, 1965a-e, 1966, 1973), and all genera accepted by Oberwinkler (2014) except Arrhytidia. The type of Arrhytidia (A. flava) was considered a species of Dacrymyces (D. marginatus), placed close to the D. chrysocomus group by McNabb (1973). No recent collections identified as such are known.

We selected eight DNA regions for phylogenetic analyses: three linked, multicopy regions in the nuclear ribosomal DNA, viz.,

1) the nuclear ribosomal small subunit (18S);

2) the internal transcribed spacer (ITS) region (including the ITS1, 5.8S, and ITS2); and

3) the D1-D3 domains of the nuclear ribosomal large subunit (28S).

We also selected three unlinked, low-copy, protein-coding, nuclear regions, viz.,

4) a c. $900-1100 \mathrm{bp}$ long fragment from the largest subunit of the DNA-directed RNA polymerase II gene between domains $A$ and $C$ (RPB1);

$5)$ a c. $1500-1700 \mathrm{bp}$ long fragment from the second largest subunit of the DNA-directed RNA polymerase II gene between domains 6 and 11 (RPB2); and

6) a c. $900-1000 \mathrm{bp}$ long fragment at the end of the translation elongation factor 1 alpha $(T E F-1 \alpha)$ gene.

Finally, we included two mitochondrial regions, viz.,

7) the mitochondrial ribosomal small subunit (12S); and

8) the ATP synthase subunit 6 (ATP6).

An outgroup was selected by retrieving from MycoCosm (Grigoriev et al. 2014) representatives from 20 of the c. 26 orders currently accepted of Tremellomycetes (three species) and Agaricomycetes (17 species). Samples were selected (Table 2) to minimise the amount of missing data in the outgroup. For each sample, nrDNA and mitochondrial regions were retrieved through BLAST searches using already generated sequences (e.g., from GenBank) of the same species or close relatives, keeping the best scoring hit. RPB1, RPB2, and TEF-1 $\alpha$ sequences were retrieved directly from the annotated contigs through the MycoCosm search option.

For morphological and cytological studies, the following fresh samples from Sweden of species also molecularly analysed were used: Dacrymyces chrysocomus s.lat. (Uppland, Uppsala, Lunsen, N59.79390 E17.68667 ${ }^{\circ}$, on Pinus sylvestris branches, 19 Nov. 2017, J.C. Zamora, UPS F-940946); D. chrysocomus s.lat. (Uppland, Solna, Järva, N59.381147 ${ }^{\circ}$ E17.998386², on Pinus sylvestris branches, 08 June 2018, J.C. Zamora, UPS F-940135); D. estonicus (Uppland, Uppsala, Lunsen, N59.79390 E17.68667 ${ }^{\circ}$, on Pinus sylvestris branches, 19 Nov. 2017, J.C. Zamora, UPS F-941253); D. stillatus (Uppland, Funbo, c. $400 \mathrm{~m}$ north of Fjällnora farm, N59.840762 ${ }^{\circ}$ E17.913327 ${ }^{\circ}$, on Pinus sylvestris branch, 14 Nov. 2017, J.C. Zamora \& S. Ekman, UPS F-941285); D. stillatus (Uppland, Solna, Hagalund, N59.361045 ${ }^{\circ} \mathrm{E} 18.029935^{\circ}$, on an old Picea abies trunk, 01 July 2018, J.C. Zamora \& I. Olariaga, UPS F-939950); D. tortus s.lat. 3 (Södermanland, Stockholm, Årsta, on Pinus sylvestris branches, 10 Sept. 2017, J.C. Zamora \& P. Posio, UPS F-941018); $D$. tortus s.lat. 4 (Södermanland, Stockholm, Masmo, N59.25429 E $17.89356^{\circ}$, on Pinus sylvestris branches, 15 Oct. 2017, J.C. Zamora \& P. Posio, UPS F-941252); D. tortus s.lat. 4 (Uppland, Solna, Bergshamra, on Pinus sylvestris branches, 23 June 2018, J.C. Zamora, UPS F-941021).

We also performed an independent and denser sampling to investigate species delimitations in Dacryonaema and Unilacryma. In total, we included DNA data of eight individuals of Da. rufum from Canada, Finland, Norway, and Sweden, 12 of Da. macnabbii from the UK (holotype), Finland, and Sweden, six of an unidentified and potentially new Dacryonaema spe- 
Table 2 Species for which DNA sequence data were retrieved from the genome database at MycoCosm (or partially GenBank), and references of the place of publication of the genomes. Data was downloaded on or prior to 1 December 2018.

\begin{tabular}{|c|c|c|c|}
\hline Class & Order & Species & References \\
\hline Agaricomycetes & Agaricales & Agaricus bisporus & Morin et al. (2012) \\
\hline Agaricomycetes & Amylocorticiales & Plicaturopsis crispa & Kohler et al. (2015) \\
\hline Agaricomycetes & Atheliales & Fibulorhizoctonia sp. & Nagy et al. (2015) \\
\hline Agaricomycetes & Auriculariales & Auricularia subglabra & Floudas et al. (2012) \\
\hline Agaricomycetes & Boletales & Suillus luteus & Kohler et al. (2015) \\
\hline Agaricomycetes & Cantharellales & Unknown (as Clavulina sp.) & Unpublished* \\
\hline Agaricomycetes & Corticiales & Vuilleminia comedens & Unpublished* \\
\hline Agaricomycetes & Geastrales & Sclerogaster hysterangioides & Unpublished* \\
\hline Agaricomycetes & Gloeophyllales & Heliocybe sulcata & Unpublished* \\
\hline Agaricomycetes & Gomphales & Ramaria rubella & Unpublished* \\
\hline Agaricomycetes & Hymenochaetales & Phellinidium ferrugineofuscum & Unpublished* \\
\hline Agaricomycetes & Jaapiales & Jaapia argillacea & Riley et al. (2014) \\
\hline Agaricomycetes & Phallales & Mutinus elegans & Unpublished* \\
\hline Agaricomycetes & Polyporales & Fomes fomentarius & Unpublished* \\
\hline Agaricomycetes & Russulales & Russula vinacea & Unpublished* \\
\hline Agaricomycetes & Sebacinales & Serendipita vermifera & Kohler et al. (2015) \\
\hline Agaricomycetes & Thelephorales & Thelephora ganbajun & $\begin{array}{l}\text { Nuclear genome unpublished* } \\
\text { Mitochondrial genome in Wang et al. (2017) }\end{array}$ \\
\hline Dacrymycetes & Dacrymycetales & Calocera cf. furcata (as Ca. cornea) & Nagy et al. (2015) \\
\hline Dacrymycetes & Dacrymycetales & Calocera viscosa & Nagy et al. (2015) \\
\hline Dacrymycetes & Dacrymycetales & Cerinomyces ceraceus & Unpublished* \\
\hline Dacrymycetes & Dacrymycetales & Dacryopinax primogenitus & Floudas et al. (2012) \\
\hline Tremellomycetes & Filobasidiales & Filobasidium wieringae & Unpublished* \\
\hline Tremellomycetes & Tremellales & Tremella mesenterica & Floudas et al. (2012) \\
\hline Tremellomycetes & Trichosporonales & Cutaneotrichosporon oleaginosus & Kourist et al. (2015) \\
\hline
\end{tabular}

* Unpublished genome data are used here with permission from their respective authors (see acknowledgements).

cies from Finland and Norway, seven of Unilacryma unispora s.lat. from Finland, Japan, and Sweden, and six of a bisporic Unilacryma from Finland and Sweden. In addition, all available GenBank sequences that had a high similarity in BLAST searches $(\geq 99 \%)$ to any of the studied DNA regions were added (Table 1). Several additional specimens of Da. macnabbii, Da. rufum, U. unispora s.lat., and the bisporic Unilacryma were collected and morphologically studied to gather a better knowledge of their intraspecific variation (see taxonomic part).

\section{Morphological study}

Whenever possible, samples were studied morphologically and photographed when fresh, either immediately after being collected or otherwise kept in a refrigerated $\left(4^{\circ} \mathrm{C}\right)$ humid chamber for a maximum of $48 \mathrm{~h}$. Alternatively, when samples were found in a dry state in the field, they were either studied directly, if the structures were well-developed, or moistened with tap water by submerging the entire substrate a few minutes, and then placed in a refrigerated humid chamber for one to seven days before study. General terminology largely follows Oberwinkler (1994), and for clamp connections we follow Stalpers (1978). In particular, 'hyphidia' is preferred over 'dikaryophyses', as the last term is not consistently applied for dikaryotic hyphidia in other groups of the Agaricomycotina, particularly in aphyllophoroid fungi. Also, we prefer the term 'young basidium' instead of 'probasidium' to avoid confusion with the thick-walled structures in Pucciniomycotina and Ustilaginomycotina, from which the basidium forms (Clemençon 2004).

Macromorphology was studied under a Leica MZ 75 dissecting microscope, and macroscopic photographs were taken with a coupled Infinity 1 macro camera. Micromorphological study was performed by mounting thin hand-cut sections of the fruitbodies in water or a $5-10 \% \mathrm{w} / \mathrm{v}$ aqueous solution of $\mathrm{KOH}$, and gently squeezing them under the cover slip. All measurements were taken at $630 \times$ magnification in $\mathrm{KOH}$ solution. The hilar appendix was not included in basidiospore measurements. Microscopic photographs were taken in a Zeiss Axio Imager A1 microscope, fitted with differential interference contrast (DIC) and equipped with an AxioCam ICc3 digital camera. For some of the most voluminous microscopic structures, a series of photographs were stacked using Combine ZP.

An aqueous solution of $1 \% \mathrm{w} / \mathrm{v}$ potassium triiodide $\left(\mathrm{KI}_{3}\right.$, Lugol's solution) was used to check the reaction of the carotenoid contents in some species. Nuclear staining was done by using toluidine blue. The best results were achieved when fresh samples were pre-treated with $5-10 \% \mathrm{KOH}$, quickly rinsed with tap water, and stained with a recently prepared alkaline solution ( $\mathrm{pH} \approx 10.5-11$ ) of $0.1 \%$ toluidine blue.

We performed analyses of variance (ANOVA) on the basidiospore length, basidiospore width, and the length/width ratio (Q) of Dacryonaema spp. and Unilacryma spp. samples, in order to detect significant differences $(p<0.001)$ among the species in each genus. Diagnostic plots for residuals (residuals vs fitted values, normal $Q-Q$, scale-location, and residuals vs leverage) were checked to ensure that no model assumptions were severely violated. Cohen's $f$ was used as an estimator of effect size. Since Dacryonaema included three species, when any significant difference was found in the ANOVA, the Tukey's honest significant difference (Tukey's HSD) post hoc test was applied to identify pairs of means different at $p<0.001$. All analyses were done using the basic package of $R$ ( $R$ Core Team 2018) and sjstats v. 0.17.4 (Lüdecke 2019). For each species, we measured 100-170 basidiospores from randomly selected fruitbodies (1-30 basidiospores per fruitbody). Measurements were taken either directly under the light microscope $(630 \times$, immersion oil), or on photographs taken as previously indicated, with the aid of Piximètre v. 5.9 (Henriot \& Cheype 2016). Length and width values were rounded to the nearest $0.1 \mu \mathrm{m}$, and $\mathrm{Q}$ values to the nearest 0.01 . 
After study, specimens were dried at room temperature and deposited in UPS. Additional herbarium specimens from $\mathrm{F}, \mathrm{K}$, NCU, O, S, TENN, UBC, and UPS were studied (herbarium acronyms following Thiers 2019). Geographical coordinates are provided in the World Geodetic System 1984 (WGS84).

\section{Lab work and primer design}

Genomic DNA was extracted from a single fruitbody or part of a fruitbody (c. $1 \mathrm{~mm}^{3}$ ), rarely (species with very small fruitbodies like Dacryonaema rufum) from 2-5 adjacent fruitbodies. The samples were disrupted in $1.5 \mathrm{ml}$ microcentrifuge tubes with a plastic micropestle either after being frozen in dried state with liquid nitrogen, or directly in the lysis buffer. We used the DNeasy Plant Mini Kit (Qiagen, Germany), following the manufacturers' instructions, but increased the lysis buffer incubation time to up to two hours. For some samples, including the type specimens of Dacrymyces macnabbii (K(M) 81678) and Unilacryma unispora (NCU F-0026842), we followed the Chelex extraction protocol of Ferencova et al. (2017), in order to minimise the amount of material used.

Forty-six different primers were used for amplifying the DNA regions mentioned above (Table 3 ), comprising both general primers used in other studies as well as newly designed primers for 12S, RPB1, RPB2, and fragments of ITS1 and ITS2 (specific primers for these last two loci designed only for Dacryonaema and Unilacryma). ITS4B (Gardes \& Bruns 1993) could not be used because the primer site has four mismatches in the genus Unilacryma, one of them affecting the last base at the 3'-end, and three mismatches in the genus Dacryonaema, in both cases resulting in unsuccessful PCR amplifications. Primer design was carried out by identifying conserved regions in Dacrymycetes alignments containing already generated sequences of those loci from MycoCosm, GenBank and own sequences obtained using general primers. We tried to design the primers so that their CG content was as close as possible to $50 \%$, with a length in the range of 17-25 bp, melting temperatures differing less than $5^{\circ} \mathrm{C}$ within a pair, and avoiding hairpin and duplex formation. Calculations were done using OligoAnalyzer v. 3.1 (Integrated DNA Technologies $®$ ).

PCR reactions were performed in a final volume of 20-25 $\mu \mathrm{l}$ using MasterMix (Qiagen, Germany). Numerous primer combinations and PCR conditions were tried for each DNA region, so we only report the most successful PCR conditions used for amplifying the majority of samples. The PCR programmes always consisted on an initial denaturation of 2 min at $94{ }^{\circ} \mathrm{C}$ and a final extension step of $7 \mathrm{~min}$ at $72^{\circ} \mathrm{C}$. The central cycles and primer pairs for the different loci greatly varied and are summarised in Table 4. When needed, nested PCRs were used for RPB1 (first PCR using DacryRPB1-1F/DacryRPB1-2r, second PCR with DacryRPB1-A/DacryRPB1-C), RPB2 (first PCR with DacryRPB2-6F/DacryRPB2-11aR, second PCR with DacryRPB2-6.2F/DacryRPB2-11bR), and TEF-1 $\alpha$ (first PCR with EF1-1018F/EF1-2218R, second PCR with Efdf/EF1-1953R).

Table 3 Primers used in this study. Within each DNA region, primers are ordered by primer site from 5' to 3', and then by increasing specificity. F = forward, $\mathrm{R}=$ reverse.

\begin{tabular}{|c|c|c|c|c|c|}
\hline Locus & Primer name & Sequence $\left(5^{\prime} \rightarrow 3^{\prime}\right)$ & Sense & Comment & References \\
\hline $18 S$ (nrSSU) & $\begin{array}{l}\text { NS1 } \\
\text { NS21UCB } \\
\text { NS4 } \\
\text { SR6 } \\
\text { NS8 }\end{array}$ & $\begin{array}{l}\text { GTAGTCATATGCTTGTCTC } \\
\text { GAATAATAGAATAGGACG } \\
\text { CTTCCGTCAATTCCTTTAAG } \\
\text { TGTTACGACTTTTACTT } \\
\text { TCCGCAGGTTCACCTACGGA }\end{array}$ & $\begin{array}{l}F \\
F \\
R \\
R \\
R\end{array}$ & & $\begin{array}{l}\text { White et al. (1990) } \\
\text { Gargas \& Taylor (1992) } \\
\text { White et al. (1990) } \\
\text { Vilgalys, unpublished } \\
\text { White et al. (1990) }\end{array}$ \\
\hline nrlTS & $\begin{array}{l}\text { ITS1F } \\
\text { ITS2 } \\
\text { ITS3 } \\
\text { ITS4 } \\
\text { ITS1DacryoUnil-F } \\
\text { ITS1Dacryo-r } \\
\text { ITS1Unil-r } \\
\text { ITS2Dacryo-F } \\
\text { ITS2Unil-F } \\
\text { ITS2DacryoUnil-r }\end{array}$ & $\begin{array}{l}\text { CTTGGTCATTTAGAGGAAGTAA } \\
\text { GCTGCGTTCTTCATCGATGC } \\
\text { GCATCGATGAAGAACGCAGC } \\
\text { TCCTCCGCTTATTGATATGC } \\
\text { CTGTTGCTGAGAAGCTGATCA } \\
\text { TTTYGTTTATGACCGCTTTCG } \\
\text { TTTGGTTACGACCGCTTGC } \\
\text { ATGCCTGTTTGAATGTCGCA } \\
\text { GAGTGTTGTGCTGTGCCT } \\
\text { CGAAGACGGTTGGAGAGC }\end{array}$ & $\begin{array}{l}F \\
R \\
F \\
R \\
F \\
R \\
R \\
F \\
F \\
R\end{array}$ & $\begin{array}{l}\text { Designed for Dacryonaema/Unilacryma } \\
\text { Designed for Dacryonaema } \\
\text { Designed for Unilacryma } \\
\text { Designed for Dacryonaema } \\
\text { Designed for Unilacryma } \\
\text { Designed for Dacryonaema/Unilacryma }\end{array}$ & $\begin{array}{l}\text { Gardes \& Bruns (1993) } \\
\text { White et al. (1990) } \\
\text { White et al. (1990) } \\
\text { White et al. (1990) } \\
\text { This study } \\
\text { This study } \\
\text { This study } \\
\text { This study } \\
\text { This study } \\
\text { This study }\end{array}$ \\
\hline $28 S$ (nrLSU) & $\begin{array}{l}\text { LR0R } \\
\text { LR3 } \\
\text { LR3R } \\
\text { LR5 }\end{array}$ & $\begin{array}{l}\text { ACCCGCTGAACTTAAGC } \\
\text { CCGTGTTTCAAGACGGG } \\
\text { GTCTTGAAACACGGACC } \\
\text { TCCTGAGGGAAACTTCG }\end{array}$ & $\begin{array}{l}F \\
R \\
F \\
R\end{array}$ & & $\begin{array}{l}\text { Cubeta et al. (1991) } \\
\text { Vilgalys \& Hester (1990) } \\
\text { Vilgalys, unpublished } \\
\text { Vilgalys \& Hester (1990) }\end{array}$ \\
\hline$T E F-1 \alpha$ & $\begin{array}{l}\text { EF1-1018F } \\
\text { Efdf } \\
\text { EF1-1953R } \\
\text { EF1-2218R }\end{array}$ & $\begin{array}{l}\text { GAYTTCATCAAGAACATGAT } \\
\text { AAGGAYGGNCARACYCGNGARCAYGC } \\
\text { CCRGCRACRGTRTGTCTCAT } \\
\text { ATGACACCRACRGCRACRGTYTG }\end{array}$ & $\begin{array}{l}F \\
F \\
R \\
R\end{array}$ & & $\begin{array}{l}\text { Stielow et al. (2015) } \\
\text { Rehner, unpublished } \\
\text { Rehner, unpublished } \\
\text { Rehner \& Buckley (2005) }\end{array}$ \\
\hline RPB1 & $\begin{array}{l}\text { DacryRPB1-1F } \\
\text { gRPB1-A } \\
\text { DacryRPB1-A } \\
\text { fRPB1-C } \\
\text { DacryRPB1-C } \\
\text { DacryRPB1-2r }\end{array}$ & $\begin{array}{l}\text { GAAGATCGAGCATTCGGAGGT } \\
\text { GAKTGTCCKGGWCATTTTGG } \\
\text { GAYTGYCCKGGRCAYTTTGG } \\
\text { CNGCDATNTCRTTRTCCATRTA } \\
\text { CRGCAATCTCGTTGTCCATGTA } \\
\text { GTTCRGGRTATGTHAGCGTCAT }\end{array}$ & $\begin{array}{l}F \\
F \\
F \\
R \\
R \\
R\end{array}$ & $\begin{array}{l}\text { Designed for Dacrymycetes } \\
\text { Improved for Dacrymycetes } \\
\text { Improved for Dacrymycetes } \\
\text { Designed for Dacrymycetes }\end{array}$ & $\begin{array}{l}\text { This study } \\
\text { Stiller \& Hall (1997) } \\
\text { This study } \\
\text { Matheny et al. (2002) } \\
\text { This study } \\
\text { This study }\end{array}$ \\
\hline RPB2 & $\begin{array}{l}\text { RPB2-6F } \\
\text { bRPB2-6F } \\
\text { DacryRPB2-6F } \\
\text { DacryRPB2-6.2F } \\
\text { fRPB2-7cF } \\
\text { bRPB2-10.9R } \\
\text { DacryRPB2-11bR } \\
\text { fRPB2-11aR } \\
\text { DacryRPB2-11aR }\end{array}$ & $\begin{array}{l}\text { TGGGGKWTGGTYTGYCCTGC } \\
\text { TGGGGYATGGTNTGYCCYGC } \\
\text { TGGGGNATGGTVTGYCCYGC } \\
\text { TTYGTSAAYGGKGTNTGGATGG } \\
\text { ATGGGYAARCAAGCYATGGG } \\
\text { GTRAASGGYGTGGCRTCYCC } \\
\text { TGRATCTTRTCRTCVACCATRTGCT } \\
\text { GCRTGGATCTTRTCRTCSACC } \\
\text { GCACGAGCRTGRATCTTRTCRTC }\end{array}$ & $\begin{array}{l}F \\
F \\
F \\
F \\
F \\
R \\
R \\
R \\
R\end{array}$ & $\begin{array}{l}\text { Improved for Dacrymycetes } \\
\text { Designed for Dacrymycetes } \\
\text { Designed for Dacrymycetes } \\
\text { Designed for Dacrymycetes }\end{array}$ & $\begin{array}{l}\text { Liu et al. (1999) } \\
\text { Matheny (2005) } \\
\text { This study } \\
\text { This study } \\
\text { Liu et al. (1999) } \\
\text { Hall, unpublished } \\
\text { This study } \\
\text { Liu et al. (1999) } \\
\text { This study }\end{array}$ \\
\hline $12 \mathrm{~S}$ (mrSSU) & $\begin{array}{l}\text { MS1 } \\
\text { DacryMS1 } \\
\text { Dacry12S-4r } \\
\text { MS2 } \\
\text { Dacry12S-2r }\end{array}$ & $\begin{array}{l}\text { CAGCAGTCAAGAATATTAGTCAATG } \\
\text { CAGCAGCTAAGAATWTTTCACAATG } \\
\text { AAYAACATRCTTCACTTCGT } \\
\text { GCGGATTATCGAATTAAATAAC } \\
\text { AGARATGGTAAGRTTTTACG }\end{array}$ & $\begin{array}{l}F \\
F \\
R \\
R \\
R\end{array}$ & $\begin{array}{l}\text { Improved for Dacrymycetes } \\
\text { Designed for Dacrymycetes } \\
\text { Designed for Dacrymycetes }\end{array}$ & $\begin{array}{l}\text { White et al. (1990) } \\
\text { This study } \\
\text { This study } \\
\text { White et al. (1990) } \\
\text { This study }\end{array}$ \\
\hline ATP6 & $\begin{array}{l}\text { ATP6-3 } \\
\text { ATP6-4 } \\
\text { ATP6-2 }\end{array}$ & $\begin{array}{l}\text { TCTCCTTTAGAACAATTTGA } \\
\text { AAGTACGAAWACWTGWGMTTG } \\
\text { TAATTCTANWGCATCTTTAATRTA }\end{array}$ & $\begin{array}{l}\mathrm{F} \\
\mathrm{R} \\
\mathrm{R}\end{array}$ & & $\begin{array}{l}\text { Kretzer \& Bruns (1999) } \\
\text { Kretzer \& Bruns (1999) } \\
\text { Kretzer \& Bruns (1999) }\end{array}$ \\
\hline
\end{tabular}


PCR products were purified using ExoSAP-IT ${ }^{\mathrm{TM}}$ (Applied Biosystems) or Illustra ${ }^{\mathrm{TM}}$ ExoProStar $^{\mathrm{TM}}$ (GE Healthcare), following the manufacturer's instructions but adding $1 / 4$ of the recommended volume of enzyme mix and extending the reaction time to 30 min. Purified PCR products were sequenced by Macrogen with the same primers used for amplification.

\section{Sequence edition and alignment}

Raw chromatograms were assembled and edited using Sequencher v. 4.1.4 (Gene Codes, USA). Even if most DNA extractions were generated from a single fruitbody, chromatograms with scattered double peaks were found in several samples. Positions with a double signal were often similar or identical among different samples (thus, from independent extractions and PCRs) of a single species, more abundant in introns and, when affecting the exons of protein coding genes, they were almost always restricted to the third codon position, resulting in alternative codons for the same amino acid (synonymous mutations). This observation suggests that more than one allele was present for a particular DNA region in some samples. For the purpose of phylogenetic reconstruction, these positions were coded using ambiguity codes. In case there was a polymorphism affecting the length of the alleles (e.g., in ITS, and often only a single base indel), the shortest copy was retained.

Two datasets were assembled and analysed independently, one for higher level taxa (overall phylogeny) and one for the species delimitation analyses of the genera Dacryonaema and Unilacryma. The ITS1 region was considered to start immediately after the highly conserved motif 'AGGACATTA' at the end of the $18 \mathrm{~S}$. The 5 ' limit of the $5.8 \mathrm{~S}$ region was defined by comparing the aligned sequences to annotated sequences from ITSoneBD (Santamaria et al. 2017). The 3'-end of the
$5.8 \mathrm{~S}$ and the 5' start of the 28S were defined using the annotate option of the ITS2 database (Keller et al. 2009). The ITS1 and ITS2 outgroup sequences were excluded for being largely unalignable among the distantly related taxa included in the dataset for the overall phylogeny. Likewise, repetitions at the end of the 28S (in Cerinomyces cf. crustulinus, C. lagerheimii, Dacrymyces flabelliformis, $D$. subantarcticensis, and Dacryopinax indacocheae) were removed, as well as two intron regions above 600 bp each in the $12 \mathrm{~S}$ (present in Vuilleminia comedens and Cerinomyces ceraceus genomic data, and in the newly generated sequences of Calocera cornea, Cerinomyces tortus s.lat. 1, and Dacrymyces cf. minutus s.lat. 2). Additionally, we excluded a highly variable and gappy region in the $12 \mathrm{~S}$ appearing between two easily alignable regions (the one in the 5' ending with the motif 'AGGGTTYGTAG', and the one in 3 ' starting with the motif 'TAGAATCW'). For the protein-coding DNA regions, intron positions were identified using open reading frames from the JGI and annotated sequences from GenBank as references (with few exceptions), and also excluded from the matrices. All sequences were trimmed to fit to the longest sequences generated by us and to remove poor read ends.

For the overall phylogeny, all alignments were estimated using PASTA v. 1.7.8 (Mirarab et al. 2015). We performed three independent runs with 10 iterations, maximum subset size being half of the sequences included in the alignment, the mask option deactivated, and keeping the best likelihood score alignment. MAFFT (L-INS-i option) was used as the aligner, OPAL as the merger, and RAxML as the tree estimator. For the ribosomal DNA regions we used the GTR $+\Gamma$ model, and for the exon regions of the protein coding genes, the WAG $+1+\Gamma$ model for the datasets temporarily translated into amino acids. The alignment with the best score after the three runs was manually

Table 4 Most successful primer combinations and central cycles of the PCR programmes.

\begin{tabular}{lll}
\hline DNA region & Primers & Central cycles \\
\hline 18S & Whole region: NS1/NS8 or NS1/SR6 & \\
& Two parts: & \\
& 1st: NS1/NS4 & (i) $5 \mathrm{cycles}$ of $45 \mathrm{~s}$ at $94^{\circ} \mathrm{C}, 45 \mathrm{~s}$ at $53^{\circ} \mathrm{C}\left(\right.$ decreasing $\left.1^{\circ} \mathrm{C} / \mathrm{cycle}\right)$, \\
& 2nd: NS21/SR6 & (ii) $35 \mathrm{cycles}$ of $45 \mathrm{~s}$ at $94^{\circ} \mathrm{C}, 45 \mathrm{~s}$ at $48^{\circ} \mathrm{C}, 1.5 \mathrm{~min}$ at $72{ }^{\circ} \mathrm{C}$
\end{tabular}

ITS+28S Both regions: ITS1F/LR5

Two parts:

ITS: ITS1F/ITS4

\section{S: LROR/LR5}

Other smaller parts: ITS1F/ITS2, ITS3/ITS4, LR0R/LR3, and LR3R/LR5

Specific for Dacryonaema/Unilacryma:

ITS1: ITS1DacryoUnil-F/ITS1Dacryo-r or ITS1Unil-r ITS2: ITS2Dacryo-F or ITS2Unil-F/ITS2DacryoUnil-r

$R P B 1$

RPB2

TEF-1a
gRPB1-A/fRPB1-C, or DacryRPB1-1F/DacryRPB1-2r, or DacryRPB1-A/DacryRPB1-C

RPB2-6F or bRPB2-6F or fRPB2-7cF/fRPB2-11aR or bRPB2-10.9R (all six combinations), or DacryRPB2-6F/ DacryRPB2-11aR, or DacryRPB2-6.2F/DacryRPB2-11bR

EF1-1018F or Efdf/EF1-1953R or EF1-2218R (all four combinations) (i) 5 cycles of $45 \mathrm{~s}$ at $94^{\circ} \mathrm{C}, 45 \mathrm{~s}$ at $55^{\circ} \mathrm{C}$ (decreasing $1^{\circ} \mathrm{C} /$ cycle), $1.5 \mathrm{~min}$ at $72^{\circ} \mathrm{C}$

(ii) 35 cycles of $45 \mathrm{~s}$ at $94^{\circ} \mathrm{C}, 45 \mathrm{~s}$ at $50^{\circ} \mathrm{C}, 1.5 \mathrm{~min}$ at $72{ }^{\circ} \mathrm{C}$ Same as above, but extension reduced to $45 \mathrm{~s}$ at $72{ }^{\circ} \mathrm{C}$ 40 cycles of $45 \mathrm{~s}$ at $94{ }^{\circ} \mathrm{C}, 45 \mathrm{~s}$ at $60^{\circ} \mathrm{C}, 30 \mathrm{~s}$ at $72{ }^{\circ} \mathrm{C}$

(i) 5 cycles of $45 \mathrm{~s}$ at $94^{\circ} \mathrm{C}, 45 \mathrm{~s}$ at $58^{\circ} \mathrm{C}$ (decreasing $1{ }^{\circ} \mathrm{C} /$ cycle), $1.5 \mathrm{~min}$ at $72{ }^{\circ} \mathrm{C}$

(ii) 35 cycles of $45 \mathrm{~s}$ at $94{ }^{\circ} \mathrm{C}, 45 \mathrm{~s}$ at $53^{\circ} \mathrm{C}, 1.5 \mathrm{~min}$ at $72{ }^{\circ} \mathrm{C}$

(i) 5 cycles of $45 \mathrm{~s}$ at $94^{\circ} \mathrm{C}, 45 \mathrm{~s}$ at $58^{\circ} \mathrm{C}$ (decreasing $1{ }^{\circ} \mathrm{C} /$ cycle), $1.5 \mathrm{~min}$ at $72^{\circ} \mathrm{C}$

(ii) 35 cycles of $45 \mathrm{~s}$ at $94{ }^{\circ} \mathrm{C}, 45 \mathrm{~s}$ at $53^{\circ} \mathrm{C}, 1.5 \mathrm{~min}$ at $72{ }^{\circ} \mathrm{C}$

(i) 5 cycles of $45 \mathrm{~s}$ at $94{ }^{\circ} \mathrm{C}, 45 \mathrm{~s}$ at $60^{\circ} \mathrm{C}$ (decreasing $1{ }^{\circ} \mathrm{C} /$ cycle), $1.5 \mathrm{~min}$ at $72^{\circ} \mathrm{C}$

(ii) 35 cycles of $45 \mathrm{~s}$ at $94{ }^{\circ} \mathrm{C}, 45 \mathrm{~s}$ at $55^{\circ} \mathrm{C}, 1.5 \mathrm{~min}$ at $72{ }^{\circ} \mathrm{C}$

(i) 5 cycles of $45 \mathrm{~s}$ at $94{ }^{\circ} \mathrm{C}, 45 \mathrm{~s}$ at $53^{\circ} \mathrm{C}$ (decreasing $1{ }^{\circ} \mathrm{C} /$ cycle), $1 \mathrm{~min}$ at $72^{\circ} \mathrm{C}$

(ii) 35 cycles of $45 \mathrm{~s}$ at $94{ }^{\circ} \mathrm{C}, 45 \mathrm{~s}$ at $48^{\circ} \mathrm{C}, 1 \mathrm{~min}$ at $72{ }^{\circ} \mathrm{C}$ 
corrected (minor modifications) and submitted to a last run in PASTA, using the parameters indicated above. Since PASTA already optimises the likelihood under a phylogenetic framework, and the resulting alignments seem to be phylogenetically accurate (Mirarab et al. 2015), neither further manual refinement was done nor parts of the alignments were excluded. All the matrices independently aligned in PASTA contained less than $15 \%$ of missing data at the ends of the sequences, except for the RPB2 dataset where c. 1/3 of the sequences (all from GenBank) were only represented by a fragment of $843 \mathrm{bp}$ covering an easily alignable exon region (a single indel due to three additional codons in the Tremellomycetes sequences).

For the Dacryonaema + Unilacryma dataset, the 5.8S, TEF-1a, and ATP6 alignments contained no indels. The RPB2 alignment contained a single, unambiguous indel of three bases in length, corresponding to a codon position. The $18 \mathrm{~S}$ and $28 S$ alignments contained only three and two unambiguous single-base indels, respectively, and were manually aligned. The Dacryonaema and Unilacryma RPB1 datasets contained a single, unambiguous codon indel and were aligned and analysed separately because the incongruence detected in the overall phylogeny (see 'Congruence among datasets' below) suggests that they may be non-orthologous. The ITS1 and ITS2 regions were independently aligned using BAli-Phy v. 3.3 (Suchard \& Redelings 2006). Since BAli-Phy is computationally intensive, we reduced the datasets by selecting two or three sequences within the same species, keeping the maximum variation but avoiding those sequences with more missing data or ambiguous base calls. A GTR $+1+\Gamma$ model was used for the substitutions, whereas an rs07 model was used for inserations and deletions. For each dataset, we executed 6 simultaneous runs with $5 \times 10^{4}$ iterations per run, checked convergence, and computed the alignments discarding the first $25 \%$ of the runs as burn-in. The average standard deviation of splits in the posterior sample turned out to be smaller than 0.005, and all parameters analysed in Tracer v. 1.7 (Rambaut et al. 2018) had effective sample sizes exceeding 1000 for individual runs ( $\geq 7000$ when combined). The resulting alignments were used as backbones for manually re-introducing the remaining sequences. Indels were coded as a separate binary dataset (gap present $=1$, gap absent $=0$ ) in FastGap v. 1.2 (Borchsenius 2009), using the simple indel coding method (Simmons \& Ochoterena 2000). All final alignments were deposited in TreeBASE (http://purl.org/phylo/treebase/ phylows/study/TB2:S24147)

\section{Congruence among datasets}

Dataset congruence was assessed for both the overall phylogeny and the Dacryonaema + Unilacryma datasets through maximum likelihood (ML) analyses using IQ-TREE v. 1.6.8 (Nguyen et al. 2015). We performed standard, non-parametric bootstrap analyses with 200 replicates for the nrDNA dataset, each of the three nuclear protein-coding genes, and the mitochondrial dataset, assuming no recombination within each dataset. Each of the protein coding genes was partitioned by codon position, the nrDNA dataset was originally divided into five partitions (18S, ITS1, 5.8S, ITS2, and 28S), and the 12S and ATP6 genes were considered as potentially different partitions. Additionally, we also tested for incongruence among partitions of the nrDNA dataset. Incongruence was considered significant when a strongly supported clade from one phylogeny was contradicted by another strongly supported clade in another phylogeny (Mason-Gamer \& Kellogg 1996). A bootstrap support (BS) threshold of $75 \%$ was used, at or above which a clade was considered strongly supported. A significant case of incongruence (Dacryonaema in the RPB1 dataset) in the overall phylogeny analyses was inspected for alternative explanations.
Possible deviations from nucleotide composition heterogeneity among taxa were checked with the IQ-TREE composition test. The amount of recombination, if any, was tested with GARD (Kosakovsky Pond et al. 2006) and SlidingBayes v. 0.94 (Paraskevis et al. 2005). Finally, we checked for heterotachy by performing analyses under the edge-unlinked partition model (Lopez et al. 2002) and the GHOST model (Crotty et al. 2017) in IQ-TREE. In the end, these processes as well as incomplete lineage sorting turned out to be unlikely candidates for the observed topological conflict, and consequently the Dacryonaema $R P B 1$ sequences were excluded from downstream analyses.

\section{Phylogenetic analyses for the overall phylogeny}

Two approaches were used for calculating phylogenies: (i) tree estimation based on the concatenated dataset of all loci; and (ii) coalescent-based species tree estimation joining independent single-locus tree phylogenies. In this set of analyses, gaps were treated as missing data. For the concatenated analyses, two strategies were used for phylogenetic reconstructions: maximum likelihood (ML) and Bayesian Inference (BI). ML analyses were performed using IQ-TREE v. 1.6 .8 (Nguyen et al. 2015), and the model selection and partitioning scheme for the mitochondrial and nuclear regions were calculated by using the Bayesian Information Criterion in the integrated version of ModelFinder (Kalyaanamoorthy et al. 2017). Fourteen potential partitions were defined for the nuclear regions: 18S, ITS1, 5.8S, ITS2, 28S, and one for each codon position of RBP1, RPB2, and $T E F-1 \alpha$, and four potential partitions were defined for the mitochondrial regions: $12 \mathrm{~S}$ and each codon position of ATP6. Branch lengths were linked and proportional across partitions. The analysis was repeated twice, starting from random trees. Branch support was assessed by standard non-parametric bootstrap, performing 500 replicates, and through the ShimodairaHasegawa-like approximate likelihood ratio test (SH-aLRT, Guindon et al. 2010), performing 1000 replicates. The purpose of using the SH-aLRT was to identify very short branches (with no signal from the data) that may have received spuriously high BS support (Guindon et al. 2010). Significant support is considered when BS $\geq 75 \%$ and SH-aLRT $\geq 85 \%$.

Bayesian inference on the concatenated dataset was carried out using MrBayes v. 3.2.6 (Ronquist et al. 2012). We applied the same partitioning scheme obtained in the previous analysis with IQ-TREE, unlinking model parameters across partitions, and using model jumping to integrate over models and their parameter values in each partition (Huelsenbeck et al. 2004), allowing a gamma distributed rate heterogeneity across sites (approximated by four categories) and a proportion of invariant sites. We used a $(1,1,1,1,1,1)$ Dirichlet prior for the substitution rates, $a(1,1,1,1)$ Dirichlet prior on the state frequencies, and a uniform $(0,1)$ prior for the proportion of invariable sites. Branch lengths were linked and proportional across partitions. We used a compound Dirichlet prior on branch lengths in the overall phylogeny $(1,0.0588,1,1)$, based on tree length estimates from the best tree of the ML analysis. We set the temperature increment parameter to 0.1 after having observed swap rates between the cold and hot chains in preliminary runs. Four parallel runs starting with random trees were executed, each with four chains, for a maximum of $1 \times 10^{8}$ generations. The analysis was automatically stopped before reaching the maximum number of generations if the average standard deviation of split frequencies dropped below 0.01. Sampling was done every 1000 th tree. The first $50 \%$ of the analysis was eliminated as burn-in, and Tracer v. 1.7 (Rambaut et al. 2018) was used to ensure that effective sample size (ESS) for each parameter exceeded 200. RWTY (Warren et al. 2017) was used to check the convergence of tree topologies. The $50 \%$ majorityrule tree with posterior probabilities $\left(\mathrm{PP}_{\text {MrBayes }}\right.$, considered signi- 
ficant when $\geq 0.95$ ) and average branch lengths was calculated from the post-burn-in trees, and visualised in FigTree v. 1.4 (Rambaut 2016).

$\mathrm{ML}$ and Bayesian approaches were also used for inferring a coalescent-based species tree from the overall phylogeny data. An ML species tree was estimated with ASTRAL III (Zhang et al. 2018, Rabiee et al. 2019) v. 5.6.3, using the independent ML phylogenetic trees obtained for each locus in IQ-TREE. Since ASTRAL uses a summary method for computing a species tree based on single-locus trees, limited accuracy is expected when the number of loci is low. Considering this limitation, we conducted two analyses, first by considering the effectively linked parts of the nrDNA as a single locus (analysis assuming five loci), and secondly by splitting the nrDNA into its units: 18S, ITS1, 5.8S, ITS2, and 28S (analysis of nine loci). The second analysis has the advantage of accounting for any unsupported discordances among those nrDNA regions, but has the shortcoming of giving too much weight to the nrDNA compared to other loci if recombination is absent among the mentioned $n r D N A$ regions. Branches receiving $\leq 10 \%$ BS support in each single-locus tree were collapsed using Newick Utilities v. 1.6 (Junier \& Zdobnov 2010), as recommended by Zhang et al. (2018). Branch support was calculated as local posterior probabilities based on quartet support (QS, Sayyari \& Mirarab 2016), and was considered significant when $Q S \geq 0.95$. Species assignments of individuals mostly followed our identifications and those from the literature (e.g., Shirouzu et al. 2009, 2017), and tended to be conservative, i.e., in some cases very closely related and potentially cryptic species might have been merged: Calocera viscosa s.lat., Dacrymyces chrysospermus s.lat., and Unilacryma unispora s.lat. In these three cases, all samples in each group formed a fully supported clade in the previous concatenated ML and Bayesian analyses. Exceptions were made when we detected either non-monophyly or a striking divergence among individuals in the $\mathrm{ML}$ analysis, e.g., the three clades of Calocera cf. guepinioides were considered as three independent species, and Dacrymyces cf. minutus s.lat. 1 and $D$. aff. stillatus, from Shirouzu et al. (2009), were treated as different species to our $D$. cf. minutus s.lat. 2 and $D$. stillatus, respectively.

StarBEAST 2, implemented in BEAST 2.5.1 (Bouckaert et al. 2014, Ogilvie et al. 2017), was used to infer a dated species chronogram for the overall phylogeny, based on the multispecies coalescent. In the absence of fossils for the included taxa, we used a secondary calibration for the Dacrymycetes, setting the stem age of the group by using a normal prior distribution with values for $\mu=350.0 \mathrm{Ma}$ and $\sigma=50.0$, according to the results from Floudas et al. (2012) (also largely agreeing with Zhao et al. 2017), under an uncorrelated lognormal relaxed clock model (Drummond et al. 2006). We used the uninformative $1 / \mathrm{X}$ prior on clock rates to allow the calibration to guide those clock rates, and a calibrated Yule tree model. Clock and tree models were unlinked among the following loci: nrDNA, RPB1, RPB2, TEF$1 \alpha$, and the mitochondrial regions. The original partitions (18S, ITS1, 5.8S, ITS2, 28S, 12S, and codon positions of each protein coding gene) were used since it is biologically implausible to combine partitions from different loci under the coalescent (e.g., codon positions of unlinked protein coding genes). We used bModelTest (Bouckaert \& Drummond 2017) for coestimating site models during the runs, averaging among all extended named models. The result of model averaging was summarised using BModelAnalyser (part of the BEAST 2 package, Bouckaert et al. 2014). Given the general life cycle in Agaricomycotina, we considered a biparental inheritance for the mitochondrion, and we used the analytical population size integration as the population model, since the number of individuals per species was low, and we were not specifically interested in per- species population sizes. Individuals were assigned to species as indicated above for the ML analysis in ASTRAL III. Initially, we executed six parallel runs starting from random trees for $5 \times 10^{8}$ generations, sampling every $10^{4 \text { th }}$ tree, then checked the traces and ESSs in Tracer v. 1.7, and discarded three of them due to failure to reach convergence for some parameters (different parameters in each run and none affecting the statistics of the species tree, see Results and Discussion). The other three runs were resumed until completing $1 \times 10^{9}$ generations. The first $50 \%$ of these three runs was eliminated as burn-in. Tracer v. 1.7 (Rambaut et al. 2018) was used to check effective sample size (ESS) of model parameters (ideally > 200). RWTY (Warren et al. 2017) was used to check the convergence of the species tree topologies. The maximum clade credibility species tree with posterior probabilities (considered significant when $\geq 0.95$ ) and node ages was calculated using TreeAnnotator v. 2.5.1 (part of the BEAST 2 package, Bouckaert et al. 2014) and visualised in FigTree v. 1.4

\section{Character evolution analyses}

We inferred the history of selected morphological characters, including transformation counts and ancestral states at the nodes corresponding to the Dacrymycetes and the four major families Cerinomycetaceae, Dacrymycetaceae, Dacryonaemataceae, and Unilacrymaceae. We avoided further analyses of characters for which convincing inferences were already provided by Shirouzu et al. (2013), and not expected to be altered by our sampling, e.g., basidiospore septation pattern, fruitbody morphology, and presence or absence of a stalk. The following traits were analysed: (i) basidium morphology $(0=2$-spored, 1 = 1-spored); (ii) clamp connections presence and morphology ( 0 = clamps absent, 1 = short and rounded clamps present at most septa, 2 = long and angulose clamps mainly restricted to the bases of basidia and hyphidia, and subhymenial hyphae); (iii) hyphidia ( 0 = conspicuous and branched, 1 = unbranched or indistinct); (iv) terminal cells of cortical/marginal hyphae ( 0 = filamentose and simple, $1=$ clearly dilated and simple, 2 = filamentose and branched); (v) carotenoids ( $0=$ absent or indistinct, 1 = conspicuous at least in some cells); (vi) cytoplasmic lipid content $(0=$ indistinct and with no visible lipid drops in the cytoplasm, 1 = lipid drops visible when the lipid bodies coalesce); (vii) number of nuclei in recently discharged basidiospores before the formation of the first septum ( $0=$ uninucleate, 1 = binucleate). Taxa with unknown states were coded as having prior probabilities equally divided among all known states. For simplicity, the character state matrix is shown next to the species tree obtained in the StarBEAST 2 analysis (Fig. 9). Our hypotheses, based on prior knowledge of the group, were as follows: (i) the two-sterigmate basidia, presence of clamps, branched hyphidia, filamentose and simple terminal cells of cortical/marginal hyphae, absence of conspicuous carotenoids, high cytoplasmic lipid content, and uninucleate young basidiospores are plesiomorphic in the Dacrymycetes; (ii) the long, angulose clamp connections at subhymenial hyphae and the bases of basidia and hyphidia, and the filamentose and branched terminal cells of cortical/marginal hyphae are plesiomorphic in the Dacryonaemataceae; (iii) binucleate young basidiospores and low cytoplasmic lipid content are acquired states plesiomorphic in the Cerinomycetaceae; (iv) the presence of carotenoids and unbranched hyphidia are acquired states plesiomorphic in the Dacrymycetaceae; (v) the 1-spored basidium, the dilated terminal cells of cortical/marginal hyphae, and the clampless septa are apomorphic conditions within different families in the Dacrymycetes.

Character transformation counts and ancestral state reconstructions were performed by stochastic mapping with SIMMAP (Bollback 2006), using the makeSimmap() function of 
the R package phytools (Revell 2012). We performed the analysis on a subsample of 1000 trees from both the posterior distribution of the MrBayes phylograms and the StarBEAST 2 chronograms to explore whether there is any difference between assuming that the phenotypic change is proportional to genetic change or to time, following Kistenich et al. (2018). The MrBayes phylograms were pruned to retain only one sample per species (the one on the shortest branch) and to exclude the outgroup. Outgroup taxa were also excluded from the StarBEAST 2 chronograms. Since the analytical approaches used for obtaining the phylogenetic trees were also different (supermatrix vs supertrees), the resulting topologies may differ as well. Under these circumstances, it may be difficult to know whether any difference in results are caused by the topology or by the scaling of branch lengths. Therefore, in case a contrasting result was found for a given character, a third group of analyses was performed using chronograms generated from the MrBayes phylograms. These chronograms were calculated using penalised likelihoods under a correlated model (Paradis 2013) with the chronos() function of the R package ape v. 5.0 (Paradis \& Schliep 2019). In all cases, ten simulations were performed for each tree. Otherwise, Kistenich et al. (2018) was followed, including the use of an instantaneous rate matrix, $Q$, estimated from the empirical data, and a prior distribution of states on the root node estimated from the stationary distribution of $Q$. However, we summarised the inferred ancestral states at the nodes of interest from all mapped trees, instead of later downsampling them, since the number of initial simulations was smaller. Reconstructions of ancestral states were focused on five nodes in the backbone of the Dacrymycetes, i.e., the crown node for the class Dacrymycetes and the four crown nodes for each named family. We found a quite obvious artefact in the reconstruction of the number of basidiospore nuclei at the root node (Dacrymycetes), which was inferred with $100 \%$ probability as binucleate with the settings indicated above. This problem was caused by an unusual calculation of the $Q$ matrix and was solved by using a symmetric model for that particular character. The problem can also be fixed by sampling $Q$ from the posterior probability distribution of $Q$ using MCMC, but this is much more computationally costly and impractical for large datasets.

Following Kistenich et al. (2018), we also checked whether ancestral state reconstructions at or near the root node were influenced by the assumptions made about the distribution of states at the root. The rayDISC() function of the corHMM v. 1.22 $R$ package (Beaulieu et al. 2013) was used for this purpose. Reconstructions were made on the pruned majority-rule consensus tree from the MrBayes analyses and on the maximum clade credibility tree from the StarBEAST 2 analysis. The same coding and state characters used for the stochastic mapping were applied. The three different assumptions about the weight given to the states at the root node allowed in rayDISC were used for calculating marginal reconstructions: (i) equal weighting among all states; (ii) based on the estimated transition rates (Yang 2006); and (iii) estimated from the data and the model (Maddison et al. 2007, FitzJohn et al. 2009).

\section{Phylogenetic and species delimitation analyses in Dacryonaema and Unilacryma}

The phylogeny of Dacryonaema and Unilacryma species based on the concatenated dataset was inferred following the same methods indicated for the overall phylogeny, i.e., $\mathrm{ML}$ analyses in IQ-TREE v. 1.6.7 and BI in MrBayes v. 3.2.6. The only differences were that we used the compound Dirichlet prior on branch lengths $(1,1.3333,1,1)$ for the Bayesian analysis, based on the different tree length estimates from the best tree of the $\mathrm{ML}$ analysis, and the temperature increment parameter was set to 0.2 , resulting in good mixing.
For the purpose of delimiting species in Dacryonaema and Unilacryma, we used the multispecies coalescent approach implemented in STACEY v. 1.2.4 (Jones 2017). Clock and tree model parameters were estimated independently for the nrDNA and each protein coding locus. We used an uncorrelated lognormal relaxed clock model (Drummond et al. 2006) and a Yule tree model. The nrDNA was divided into three partitions, (i) $18 \mathrm{~S}+5.8 \mathrm{~S}+28 \mathrm{~S}$, (ii) ITS1 + ITS2 and (iii) coded indels, and each protein coding locus was divided in (i) $1^{\text {st }}+2^{\text {nd }}$ codon positions, (ii) the $3^{\text {rd }}$ codon position, and (iii) coded indels for each locus, if present. With this simplified scheme we tried to avoid overpartitioning, since the amount of variation in the Dacryonaema + Unilacryma dataset was relatively low, particularly the first and second codon positions of the protein coding genes, and parameter estimates when treating those partitions independently were sometimes poor in preliminary analyses. Parameters of the site models were independently estimated for each DNA partition using bModelTest (Bouckaert \& Drummond 2017), taking into consideration all transition/transversion split models, while the Lewis MK model with two states was used for the binary partitions. The most visited models were summarised using BModelAnalyser (part of the BEAST 2 package, Bouckaert et al. 2014). We used the CIPRES Science Gateway (Miller et al. 2010) to run ten MCMC parallel analyses for $1 \times 10^{9}$ generations, sampling every $5 \times 10^{4 \text { th }}$ tree, and with the collapse height parameter $\varepsilon=10^{-4}$. Considering the amount of missing data (most individuals from GenBank were represented by a single locus) and the taxonomic information from both morphology and the phylogenetic analyses on the concatenated dataset, we used an informative prior for the collapse weight parameter $(\omega)$. Since the most likely number of species according to the tree topology and information from morphology was around five or six, and impossible below two (that would imply considering all samples of Dacryonaema and Unilacryma not only as a single genus, but as a single species), we set $\omega=0.908$ (highest probability around $k=5-6$ ), with 0.5 $(\mathrm{k}=25)$ and $0.98(\mathrm{k}=2)$ as the lower and upper values of the interval, respectively. The first half of each run was discarded as burn-in, and the remaining posterior sample was used to calculate the most likely number of clusters (i.e., putative species), using SpeciesDelimitationAnalyzer (Jones et al. 2014). The matrix of pairwise posterior probabilities of belonging to the same cluster was subsequently visualised and plotted in R (R Core Team 2018) following Jones et al. (2014). The chronogram showing the phylogenetic position of all included samples was drawn with FigTree v. 1.4.

\section{RESULTS}

\section{Morphological results}

\section{Vacuolar cell contents and its variation with mounting media}

The cytoplasm of living cells in all studied species of Dacrymycetes was found to be filled with conspicuous, highly refractive vacuolar bodies (VBs) that are variable in size and shape within specimens but not between species (Fig. 3a, c, g, i, m, $\mathrm{o}, \mathrm{s}, \mathrm{u})$. The VBs disappear when treated with alkaline lethal media, such as $\mathrm{KOH}$, or when cells die by other causes, e.g., desiccation (Fig. 3b, d-f, h, j-l, n, p-r, t, v-x).

Lipid bodies (LBs) have been observed in nearly all studied samples but, unlike VBs, they show considerable variation. Conspicuous LBs are always absent in living cells, being reduced to hardly discernible vacuoles of granular appearance $(\leq 0.2 \mu \mathrm{m}$ diam, unaffected by $\mathrm{KOH}$ treatment) due to the presence of the much more conspicuous VBs. When cells die, the LBs become more visible and tend to gradually fuse, forming larger oil drops when the amount of LBs allows so. The difference in 


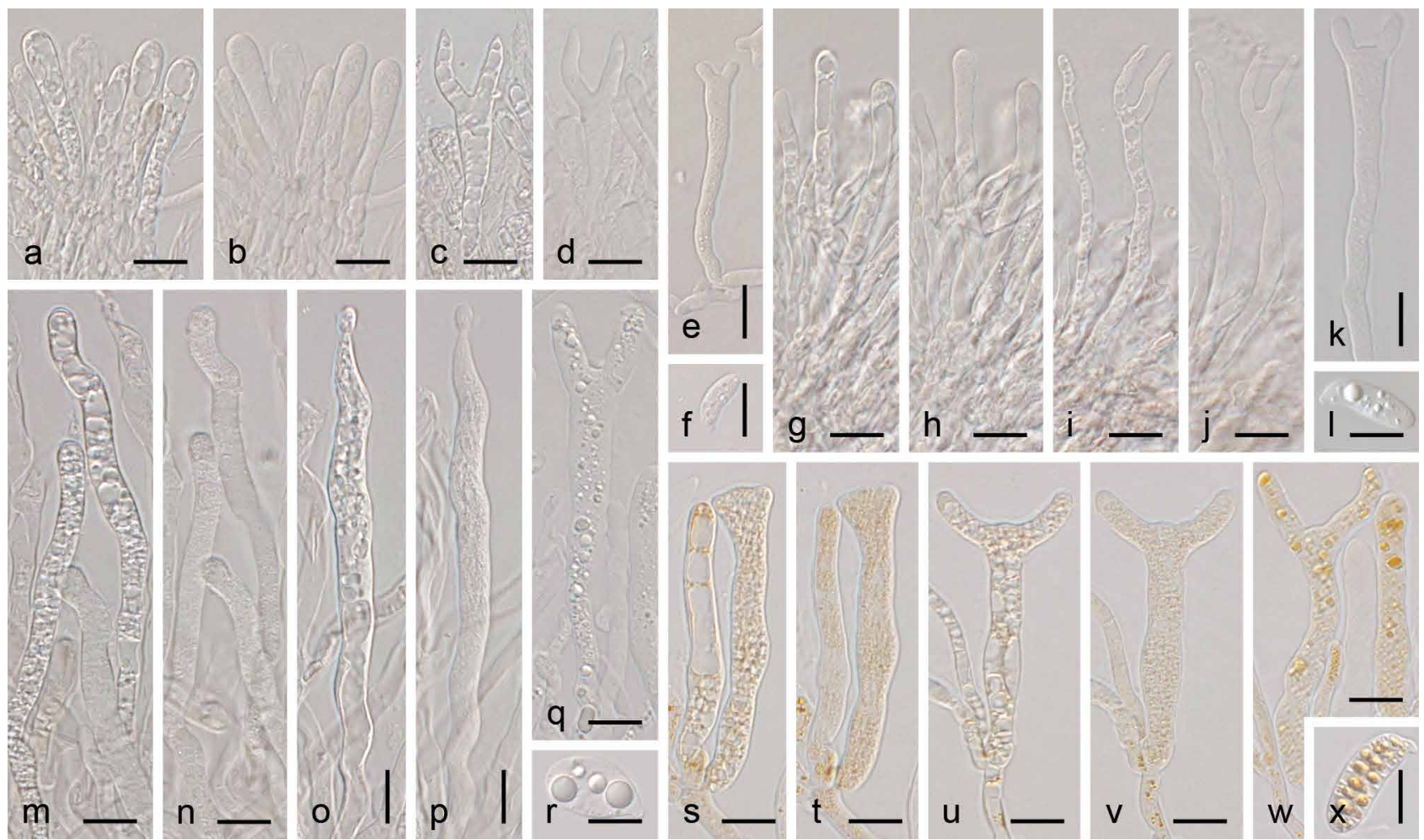

Fig. 3 Vacuolar cells contents in the Dacrymycetes. a-d, g-j, m-p, s-v. Vacuolar contents in alive (mounted in water) and recently killed (after adding $10 \% \mathrm{KOH}$ ) young and mature basidia; $\mathrm{e}-\mathrm{f}, \mathrm{k}-\mathrm{l}, \mathrm{q}-\mathrm{r}, \mathrm{w}-\mathrm{x}$. vacuolar contents in dead and relatively old basidia and basidiospores after coalescence of the lipid bodies, if present. a-d. Dacrymyces tortus s.lat. 4 (UPS F-941021); e-f. D. tortus s.lat. 3 (UPS F-941018); g-j. Dacryonaema rufum (UPS F-941006); k. Da. macnabbii (UPS F-940954); I. Da. macrosporum (UPS F-941001); m-p. Unilacryma unispora (UPS F-941286); q-r. U. bispora (holotype); s-v. Dacrymyces chrysocomus s.lat. 2 (UPS F-940135); w-x. D. chrysocomus s.lat. 2 (UPS F-940946). - Scale bars $=10 \mu \mathrm{m}$.

the amount of visible cytoplasmatic lipid content among groups is more pronounced in basidiospores, less so in basidia, and even less in somatic cells.

In Cerinomycetaceae, the cytoplasm of samples treated with $\mathrm{KOH}$ appears either as undifferentiated or, at most, as only slightly granular, with an inconspicuous lipid content (Fig. 3b, $\mathrm{d}-\mathrm{e}$ ). Big oil drops ( $>2 \mu \mathrm{m}$ diam) are rarely seen in dead cells of samples of this family (Fig. 3f), neither in rapidly desiccated samples nor in samples exposed to reagents that favour the coalescence of LBs, such as ethanol or $\mathrm{HCl}$. However, exceptions occur, and $D$. tortus s.lat. 1 displayed a rather high lipid content, comparable to some species in the Dacrymycetaceae.

In Dacryonaemataceae, there is a large variation among the three species (Fig. 3h, j-I). Dacryonaema rufum has a comparatively low cytoplasmic lipid content, and LBs are not visible or hardly discernible in dead cells, even after forcing their coalescence (Fig. 3h, j). Dacryonaema macnabbii and especially Da. macrosporum may show a considerable amount of LBs, and some big lipid drops may form when the LBs coalesce (Fig. 3k-l).

In all studied species of Dacrymycetaceae and Unilacrymaceae the cytoplasm of various cells (particularly in basidia and basidiospores) appears clearly granular when samples are mounted in $\mathrm{KOH}$ (Fig. 3n, p, t, v). In addition, a few big oil drops (> $2 \mu \mathrm{m}$ diam) are commonly observed in at least some of the dead cells (Fig. $3 q-r, w-x$ ).

Coalesced LBs are often visible in well-preserved herbarium samples, even decades after being collected. However, the cytoplasm tends to become rather uniformly refractive with age in some old herbarium specimens, in which case internal details are lost.

\section{Other variations with mounting media}

Cell size and shape displayed little variation among living or dead cells mounted in water and dead cells mounted in $\mathrm{KOH}$ (Fig. 3). On the other hand, due to the commonly large size of many structures and the elasticity of the cell walls, dimensions are easily altered by simply applying pressure on the cover slip, or by premature mounting of insufficiently rehydrated specimens. The terminal cells of cortical/marginal hyphae in Dacryonaema and a few other genera (e.g., Heterotextus) are an exception, since walls become distinctly gelatinised and expanded in $\mathrm{KOH}$.

Hyphae with somewhat roughened walls (e.g., some internal hyphae in various species, Fig. 2d) are often more distinctly roughened when the sections are mounted in $\mathrm{KOH}$, perhaps due to the precipitation of some substances of the intercellular gelatinous matrix.

\section{Nuclei}

Nuclei are masked by the VBs in the living state, and are best studied in recently dead cells. In species with highly pigmented cytoplasms, nuclei appear as 'empty' areas due to the absence of pigments, and are relatively easy to observe in fresh samples mounted in $\mathrm{KOH}$ (Fig. 4c, e-g, k-m). They are also observable under the same conditions in species with a very low amount of lipid contents (Fig. 4j, n, q). Nuclei can sometimes be seen in slides prepared with Lugol's solution as slightly paler areas of the cytoplasm when the cells are still alive or have recently died (Fig. 4t). Sometimes, they appear as slightly darker areas (due to staining) when the cells are dead. The best contrast is often achieved by using alkaline toluidine blue solutions, where the nuclei (or at least the nucleoli) stain deeply blue, while the cytoplasm stains less intensely and often more purplish, depending on the species and small variations in $\mathrm{pH}$ (Fig. $4 \mathrm{a}-\mathrm{b}$, $\mathrm{d}, \mathrm{h}-\mathrm{i}, \mathrm{o}-\mathrm{p}, \mathrm{r}-\mathrm{s}, \mathrm{u}-\mathrm{v}$ ). 


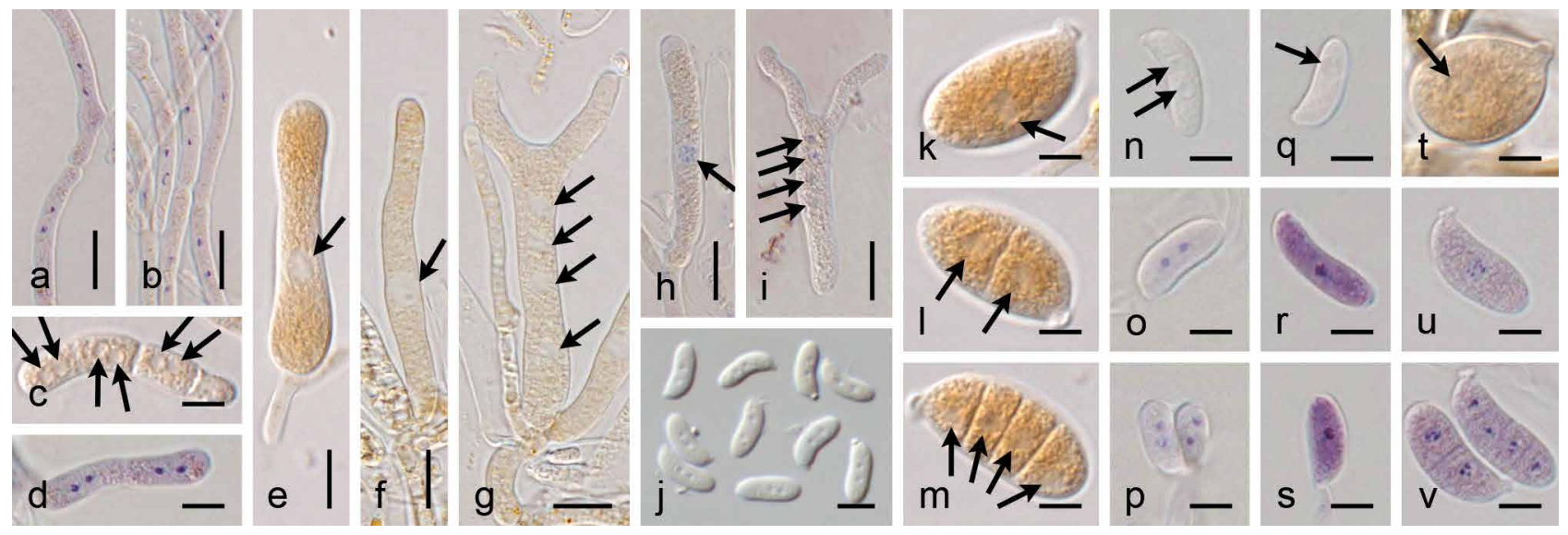

Fig. 4 Nuclei in some cells in the Dacrymycetes. a-b. Dikaryotic vegetative hyphae; c-d. dikaryotic arthrospores; e-f, h. young basidia showing the single diploid nucleus after cariogamy; g, i. basidia showing the four post-meiotic haploid nuclei; j, $n-p$. binucleate basidiospores; k-m, u-v. basidiospores showing a single nucleus when aseptate and then one nucleus per cell after septa are formed; $q-s$. aseptate basidiospores with a single nucleus; t. basidiospore with a slightly visible (slightly paler) nucleus. a-b, d, h-i, o-p, r-s, u-v. Stained with toluidine blue; c, e-g, j-n, q. in KOH solution; t. stained with Lugol's solution. a-d, h-i, u-v. From Dacrymyces stillatus (UPS F-939950); e, k-m. from D. estonicus (UPS F-940137); f-g. from D. chrysocomus s.lat. (UPS F-940135); j. from Dacrymyces tortus s.lat. 3 (UPS F-941016); n-p. from D. tortus s.lat. 4 (UPS F-941021); q. from Dacryonaema rufum (UPS F-941006); r-s. from Da. macnabbii (UPS F-940954); t. from Unilacryma unispora (UPS F-941284). — Scale bars: $a-b, e-j=10 \mu m, c-d, k-v=5 \mu m$.

Vegetative cells in all fresh samples studied are dikaryotic, and mitospores formed directly from those hyphae, e.g., the arthrospores of Dacrymyces stillatus are dikaryotic as well (Fig. $4 a-d)$. Basidia under formation are originally dikaryotic, but the two haploid nuclei quickly fuse into a single, large, diploid nucleus (Fig. $4 \mathrm{e}-\mathrm{f}, \mathrm{h}$ ). At a certain state of development, often when the sterigmata are already formed, meiosis occurs and sometimes the four postmeiotic nuclei are revealed (Fig. $4 \mathrm{~g}, \mathrm{i}$ ), the position of which varies within the mature basidium.

In Cerinomycetaceae, young basidiospores constantly have two nuclei, even when still attached to the basidium (Fig. $4 \mathrm{j}, \mathrm{n}-\mathrm{p}$, observed also in two additional species not included in phylogenetic analyses).

In Dacrymycetaceae and Dacryonaemataceae, a single nucleus passes into each basidiospore and is not immediately followed by another nuclear division, resulting in young basidiospores that are always uninucleate (Fig. 4k, q-s, u, observed also in c. 15 additional Dacrymycetaceae species not included in the phylogenetic analyses). After discharge, they remain uninucleate until mitosis occurs (including septation), and septate basidiospores contain a single nucleus in each daughter cell (Fig. 4I-m, v). This has been clearly observed in basidiospores with up to eight cells. When the number of cells is higher, nuclei are obscured by the narrowness of each individual cell. Scattered binucleate, aseptate basidiospores have been seen in samples where several other basidiospores were already septate, probably representing a developmental stage immediately prior to septation.

In Unilacrymaceae, nuclei are very difficult to observe in the basidiospores due to the combination of large volume, high content of lipid bodies, and absence of a colourful cytoplasm (see 'pigments' below). In addition, the differential staining of nuclei with toluidine blue is not as effective as in samples of the other families. Nevertheless, our observations indicate that basidiospores are very likely uninucleate prior to septation (Fig 4t).

Nuclear details are often lost some time after desiccation, except typically for xerotolerant species (e.g., in Dacryonaema), where nuclei can be visible at least in the basidiospores for longer periods after being collected.

\section{Pigments}

There is considerable colour variation in the fresh fruitbodies of the four families of the Dacrymycetes. Fruitbodies in the
Dacrymycetaceae are often yellow or orange (Fig. 5d), although other colours, e.g., brownish or whitish, occur. In the Cerinomycetaceae, Dacryonaemataceae, and Unilacrymaceae duller colours, e.g., pale cream, ochre, brown or grey, are the rule (Fig. $5 a-c$ ), although young fruitbodies of some species may be rather orange. The spore print is often yellow to orange in many species of Dacrymycetaceae with also yellow to orange fruitbodies (Fig. 5e), pale cream or whitish in paler species and in all samples of Cerinomycetaceae, Dacryonaemataceae and Unilacrymaceae from which we were able to obtain a spore print.

Yellow-orange carotenoid pigments were observed mainly in the lipid bodies of the cytoplasm (Fig. 5f, i-l, p-q, s). In some species, these pigments are only clearly observable after having been concentrated by the coalescence of the lipid bodies and are easily overlooked in living or recently dead cells (Fig. $5 p-q)$. A distinct carotenoid content was observed in most fresh specimens of Dacrymycetaceae and also in some herbarium samples. In Calocera cornea, the yellowish colour may be particularly inconspicuous and is best seen in the basidium cytoplasm. In Unilacrymaceae and Da. macrosporum, carotenoids are typically not visible (Fig. $5 r$ ), but an inconspicuous yellowish cream tinge (never bright yellow or bright orange) after the fusion of lipid bodies may be seen in fruitbodies growing in very bright places and receiving more sun-light. In Cerinomycetaceae, Da. rufum, and Da. macnabbii, yellow or orange colours were not discernible within the cytoplasm and, as noted above, no big oil drops that could concentrate these pigments are normally formed when the cells die (Fig. $5 n-0$ ). These observations do not imply that Cerinomycetaceae, Dacryonaemataceae, and Unilacrymaceae are necessarily devoid of carotenoids, but indicate that the amount of carotenoids, if present, is distinctly lower than in most species of Dacrymycetaceae, or of a different composition. The carotenoids progressively degrade after the samples are collected. For this reason, the yellow or orange colours tend to slowly fade in herbarium specimens and may disappear after a few decades.

The carotenoid contents in the studied species of Dacrymycetaceae show a distinct greenish to greenish grey reaction with Lugol's solution (Fig. $5 \mathrm{~g}, \mathrm{~m}$ ), although in Calocera cornea it is often inconspicuous (it may be seen on spore prints). This reaction has not been observed in any species of Cerinomycetaceae, Dacryonaemataceae, or Unilacrymaceae (Fig. 5h), 


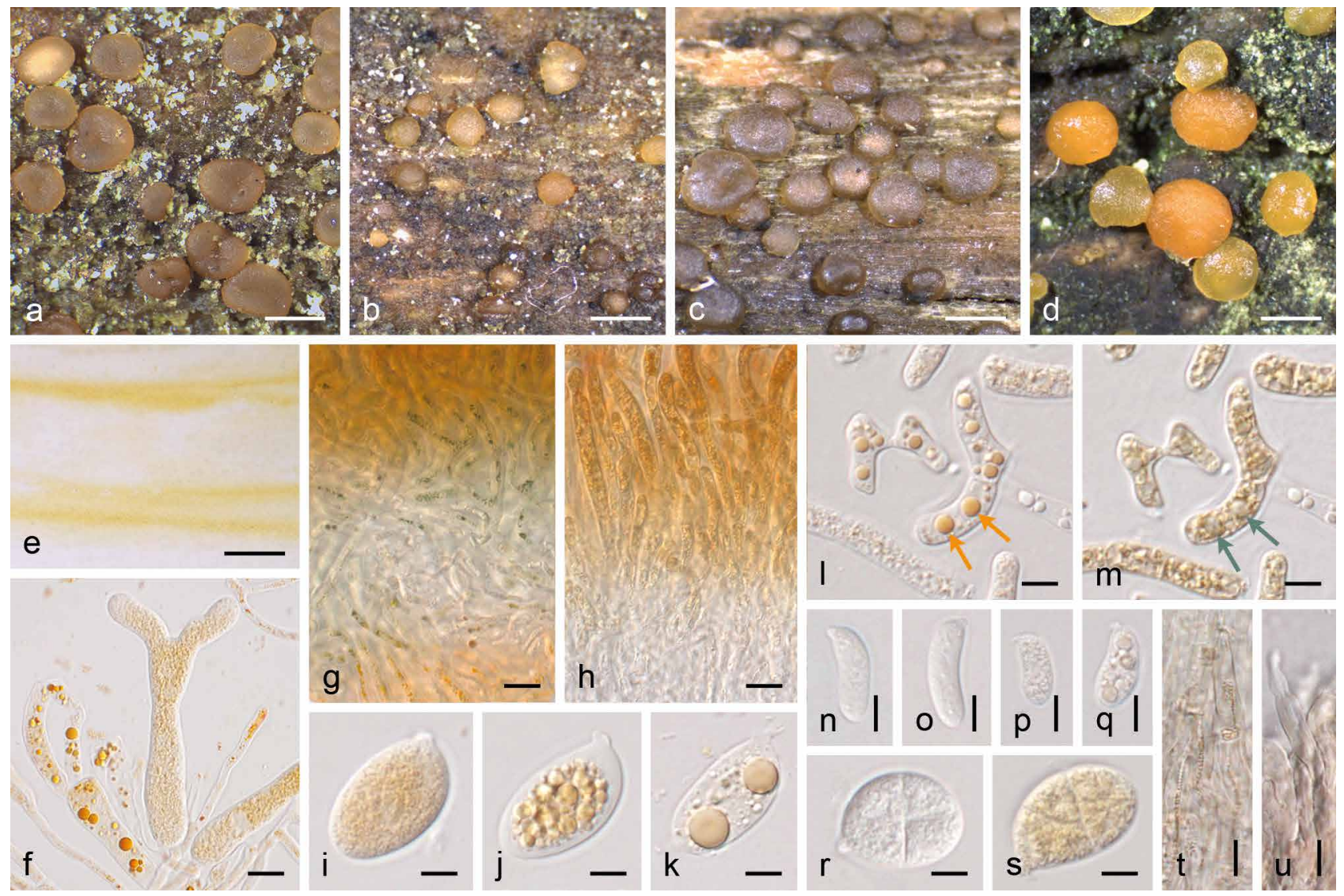

Fig. 5 Pigments in the Dacrymycetes. a-d. Representative species with pulvinate and sessile ('dacrymycetoid') fruitbodies in the Dacryonaemataceae (a. Dacryonaema macnabbii, UPS F-940954), Unilacrymaceae (b. Unilacryma unispora, UPS F-941284), Cerinomycetaceae (c. Dacrymyces tortus s.lat. 3, UPS F-941018), Dacrymycetaceae (d. D. stillatus, UPS F-939816); e. yellow-orange spore print of Calocera viscosa (UPS F-940773); f, i-k. basidia and basidiospores of $D$. estonicus (UPS F-940137); $\mathrm{g}-\mathrm{h}$. reaction of the carotenoid contents with Lugol's solution when applied from above to cuttings of $D$. estonicus (g, UPS F-940137) and U. unispora (h, UPS F-941284); I-m. dead arthrospores of $D$. stillatus (UPS F-941285) in water and after the application of Lugol's solution, arrows depict the change in colour of lipid drops with carotenoids; $\mathrm{n}-\mathrm{o}$. basidiospores of Dacryonaema rufum (n, UPS F-941005) and Dacrymyces tortus s.lat. 4 (o, UPS F-941252); p-q. basidiospores of Calocera viscosa (UPS F-940773), before (p) and after (q) the coalescence of the lipid bodies; $r-s$. similarly shaped but differently pigmented basidiospores of Unilacryma unispora (r, UPS F-941280) and Dacrymyces ovisporus (s, UPS F-940139); t. cortical/marginal hyphae in Dacryonaema macrosporum (UPS F-941000), with brownish intracellular pigments; u. marginal hyphae in Da. rufum (UPS F-941005) with diffuse, brownish parietal pigment. - Scale bars: $\mathrm{a}-\mathrm{e}=1 \mathrm{~mm}, \mathrm{f}-\mathrm{h}=10 \mu \mathrm{m}, \mathrm{i}-\mathrm{u}=5 \mu \mathrm{m}$

not even when a pale yellowish cream tinge was present in the cytoplasm

Additional pigments, both intracellular and parietal, are sometimes seen in various species of all four families. An intracellular brownish pigment, seen as small granules in water but partially soluble in $\mathrm{KOH}$, has been noted in samples of all three species of Dacryonaema, and it is particularly conspicuous in some marginal cells of Da. macrosporum (Fig. 5t). Parietal diffuse pigments were observed in Dacrymyces tortus s.lat. and all species of Dacryonaema and Unilacryma, in Dacryopinax elegans, and in a few Dacrymyces s.lat. species not included in the present phylogenetic analyses. They are more conspicuous in the sterile marginal cells and may vary from clearly brownish to yellowish brown, brownish orange, or reddish brown. In Dacryonaema, they are sometimes better distinguished from the intracellular pigments when mounting samples in $\mathrm{KOH}$ (Fig. 5u). These pigments seem to provide at least part of the dark colour in species like Da. rufum, and are more persistent than the carotenoids in herbarium specimens. All pigments tend to be more abundant in fruitbodies growing in sun-lit places.

\section{Dacryonaema and Unilacryma morphometric analyses}

A total of 625 basidiospores were measured (375 for Dacryonaema spp. and 250 for Unilacryma spp.). ANOVA analyses of the three measured characters (basidiospore width, basidiospore length, and basidiospore Q, Fig. 6) showed significant differences in basidiospore width and $Q$ in the Unilacryma dataset $(p<0.001$, Cohen's $f>0.97)$, but not in basidiospore length ( $p=0.011$, Cohen's $f=0.163$ ), while there were significant differences among the three species of Dacryonaema in all three characters $(p<0.001$, Cohen's $f>1.66$ for basidiospore length and width, and Cohen's $f=0.496$ for basidiospore $Q$ ). Further analysis by Tukey's HSD test on the Dacryonaema dataset found significant differences $(p<0.001)$ in all pairwise comparisons except between Da. macnabbii and Da. rufum basidiospore length $(p=0.002)$ and $D a$. macrosporum and Da. rufum basidiospore $Q(p=0.0014)$. Dacryonaema macrosporum has longer and wider basidiospores than $D a$. macnabbii and Da. rufum. Dacryonaema macnabbii basidiospores are also wider than those of Da. rufum. Dacryonaema macnabbii has basidiospores tending to be ellipsoid to cylindric-allantoid, whereas those of Da. macrosporum and Da. rufum are cylindric to narrowly cylindric-allantoid. Unilacryma bispora basidiospores are significantly narrower than $U$. unispora basidiospores, which is reflected in the significantly different $Q$, most basidiospores being narrowly ovoid to ellipsoid in $U$. bispora and subglobose to ovoid in U. unispora.

\section{Molecular results}

\section{Molecular data}

Counting each of the three parts of the nrDNA as independent sequences, 402 new sequences were generated in this study 
Dacryonaema

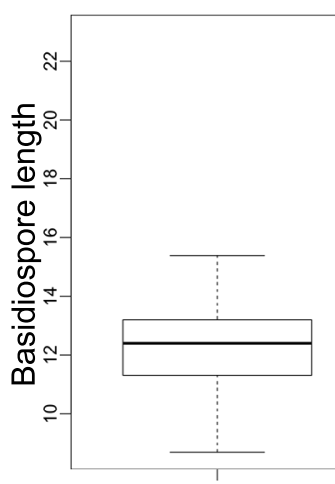

Da. macnabbii Da. macrosporum $p<0.001$ : Da. macrosporum with $D$. macnabbii and $D a$. rufum $p=0.002$ : Da. macnabbii-Da. rufum

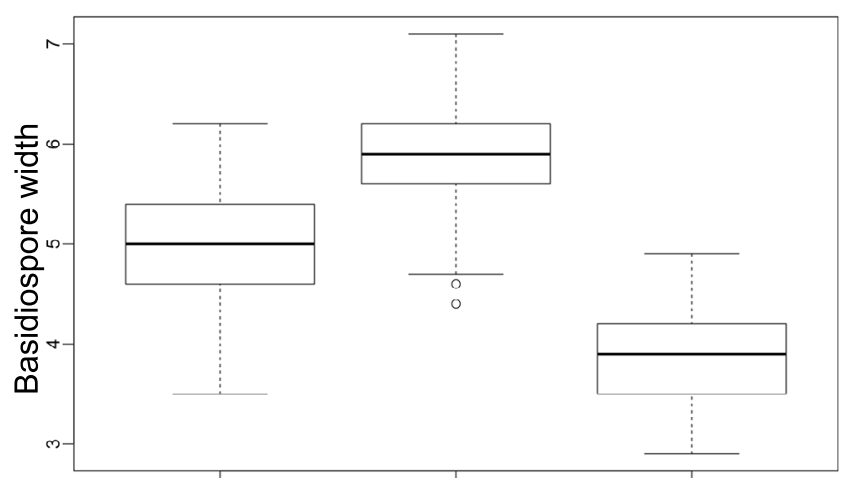

Da. macnabbii Da. macrosporum $p<0.001$ : all, Cohen's $f=1.678$

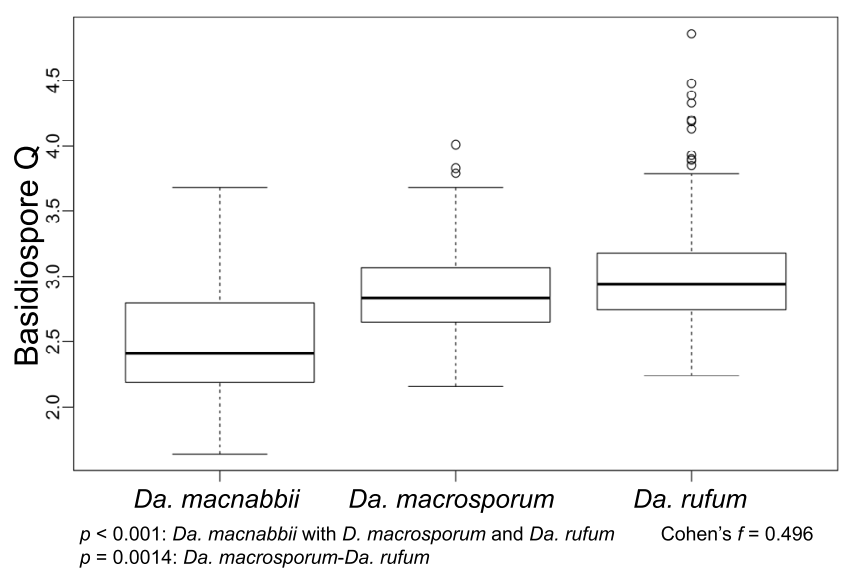

Fig. 6 Morphometric comparisons of Dacryonaema spp. and Unilacryma spp.

(Table 1). The final dataset for the overall phylogeny included 188 individuals (20 in the outgroup and 168 in the ingroup) that represent around 110 putative species (three in Tremellomycetes, 17 in Agaricomycetes, and c. 90 in Dacrymycetes). The combined matrix consisted of 8890 characters (767 12S, 694 ATP6, 1715 18S, 282 ITS1, 171 5.8S, 296 ITS2, 947 28S, 1155 $R P B 1,1848$ RPB2, $1015 T E F-1 \alpha)$, of which 4029 were variable and parsimony informative (323 12S, 462 ATP6, 392 18S, 158 ITS1, 57 5.8S, 224 ITS2, 389 28S, 644 RPB1, 971 RPB2, 409 TEF-1 $\alpha$ ) and 718 variable and parsimony uninformative (90 12S, 61 ATP6, 100 18S, 45 ITS1, 10 5.8S, 20 ITS2, 76 28S, 88 RPB1, 170 RPB2, 58 TEF-1 $\alpha$ ).

The final dataset for the Dacryonaemataceae + Unilacrymaceae analyses included 50 terminals. The combined matrix consisted of 7796 characters (638 ATP6, 1653 18S, 146 ITS1, 154 5.8S, 292 ITS2, 886 28S, 700 RPB1 (Dacryonaema), 697 RPB1 (Unilacryma), 1620 RPB2, 913 TEF-1a, 97 indels), of which 1283 were variable and parsimony informative (163 ATP6, 36 18S,
Unilacryma

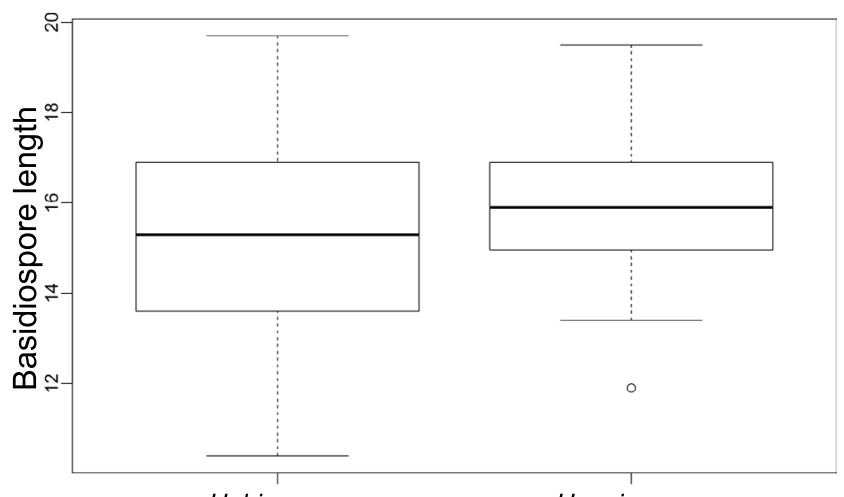

U. bispora

$p=0.011$, Cohen's $f=0.163$

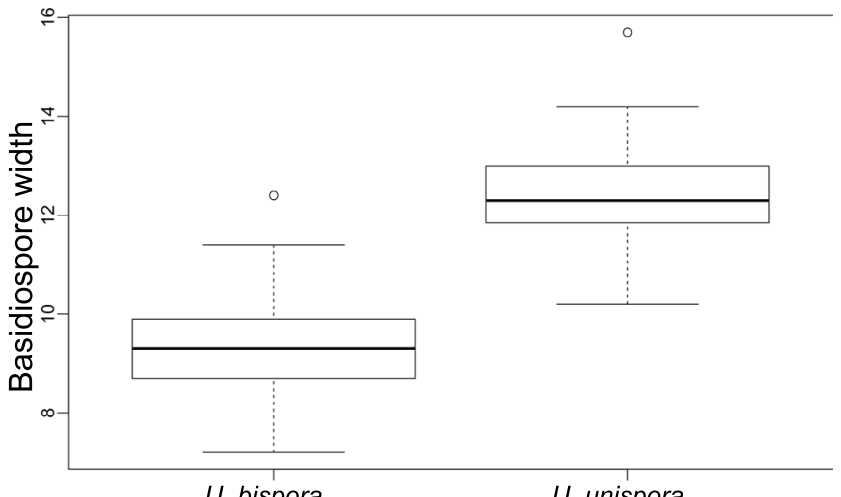

$p<0.001$, Cohen's $f=1.678$

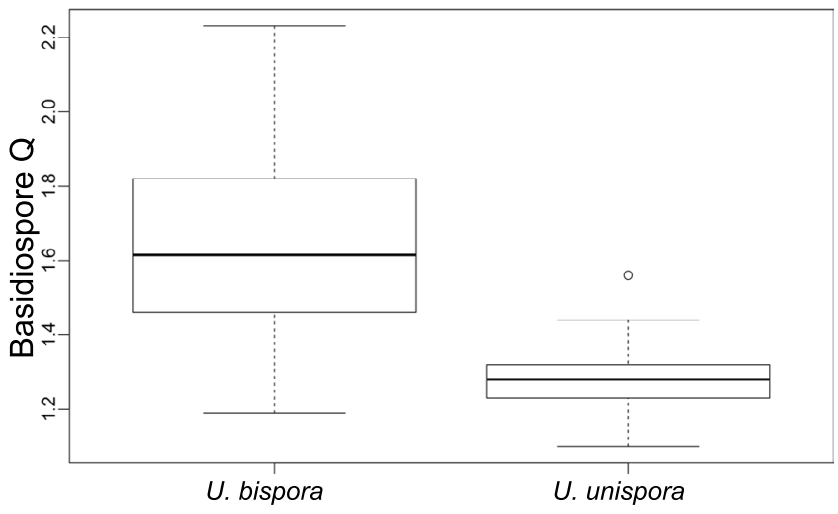

$p<0.001$, Cohen's $f=0.972$

30 ITS1, 8 5.8S, 37 ITS2, 65 28S, 61 RPB1 (Dacryonaema), 154 RPB1 (Unilacryma), 490 RPB2, 154 TEF-1a, 85 indels) and 167 variable and parsimony uninformative (0 ATP6, 1 18S, 1 ITS1, 0 5.8S, 4 ITS2, 9 28S, 66 RPB1 (Dacryonaema), 6 RPB1 (Unilacryma), 55 RPB2, 13 TEF-1 $\alpha, 12$ indels).

No introns were detected in the nrDNA or in the ATP6 sequences of any of the included species. Five introns were identified in the amplified RPB1 fragment. All species in Dacrymycetes lack the third RPB1 intron (spliceosomal intron 2 in Matheny et al. 2002), which is consistently present in all species in the outgroup. All species included in the study have a single intron in RPB2 at domain 7 (Arabidopsis intron 16).

In $T E F-1 \alpha, 12$ introns were identified in the alignment, seven of them exclusively present in the Tremellomycetes. The second closest intron to the 5'-end is present in some lineages of the Dacrymycetes (Dacryonaemataceae, Unilacrymaceae, Cerinomycetaceae, and the $D$. chrysocomus clade), as well as in all included species of Agaricomycetes and in Filobasidium 

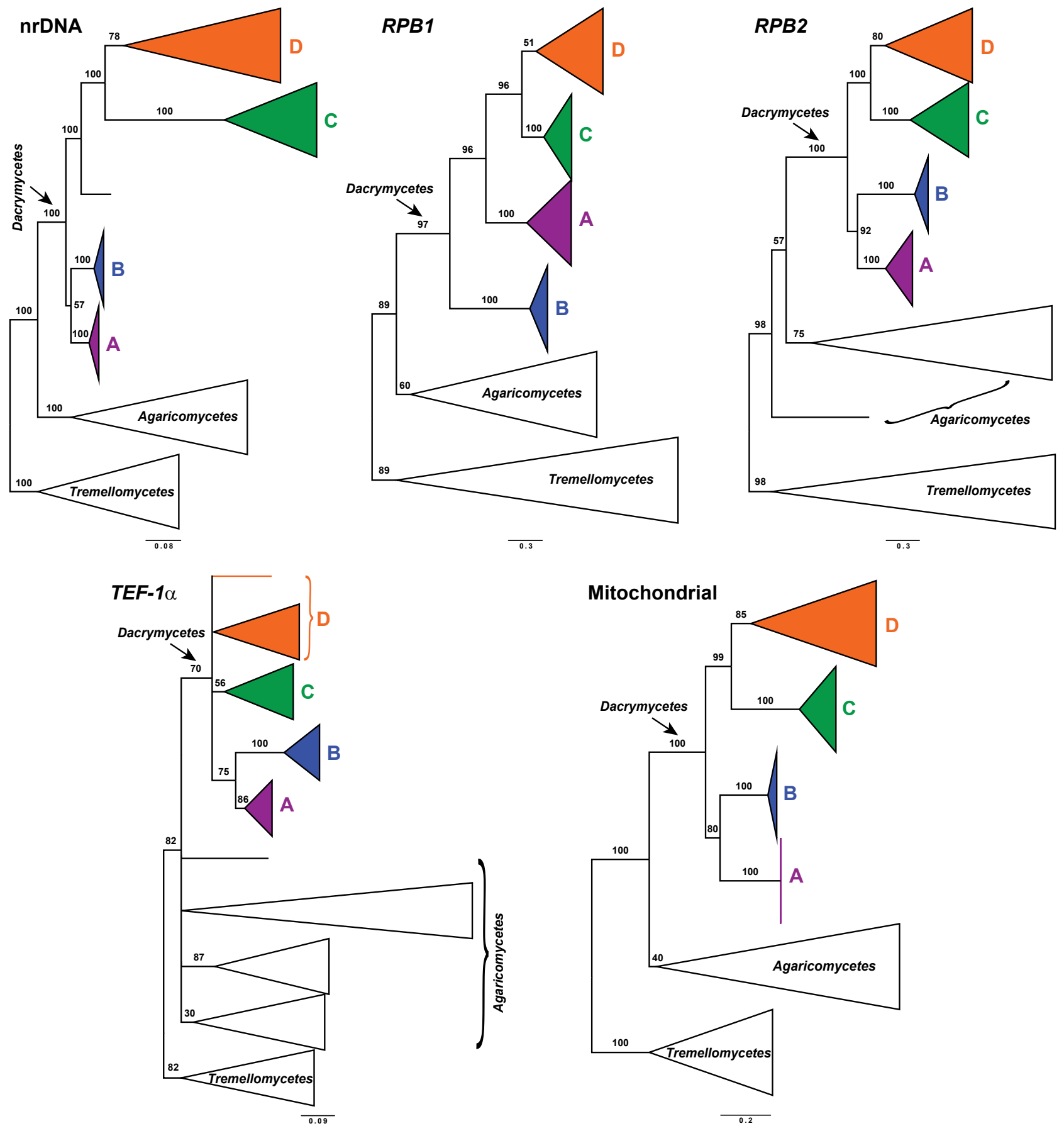

Fig. 7 Maximum likelihood phylogenetic trees from each DNA locus including all samples for the overall phylogeny. A = Unilacrymaceae, B = Dacryonaema, $C=$ Cerinomycetaceae, $\mathrm{D}=$ Dacrymycetaceae. Numbers on branches indicate ML bootstrap support. Branches with BS $\leq 20 \%$ are collapsed for clarity. Note the incongruent phylogenetic position of the Dacryonaema clade in the RPB1 phylogram, sister to all other Dacrymycetes with high support.

wieringae. It is absent in most Dacrymycetaceae species and in Tremella mesenterica and Cutaneotrichosporon oleaginosus. The third intron in TEF-1a is present in Dacryonaemataceae, Unilacrymaceae, and Cerinomycetaceae, and absent in all Dacrymycetaceae. In the outgroup, it is present in most species except Plicaturopsis crispa and Filobasidium wieringae. The sixth intron is only present in Dacryonaemataceae within the Dacrymycetes, and its presence or absence varies in the outgroup, appearing in about $2 / 3$ of the included species.

Three introns were identified in $12 \mathrm{~S}$. The first intron is exclusively present in some Dacrymycetes species (Calocera cornea, Cerinomyces ceraceus, Cerinomyces tortus s.lat. 1, and Dacrymyces cf. minutus s.lat. 2) and was located in the motif 'AACAGGAT[I]TWGRKACCCAGG', where '[I]' denotes the intron. The Vuilleminia comedens sequence had another exclusive intron (over $1.5 \mathrm{~kb}$ ) in the motif 'GCAAARBT[I]GAAAWCAAAAAAA'.
In both Calocera cornea sequences, a third intron was present at the end of the sequence, probably immediately before the primer site for Dacry12S-2r.

\section{Overall phylogeny}

Preliminary analyses of all data showed a significant incongruence in the RPB1 gene tree involving the placement of Dacryonaema. RPB1 data placed Dacryonaema as sister to all other Dacrymycetes with high support (BS = $96 \%$ for the clade sister to Dacryonaema and $100 \%$ for the Dacryonaema clade itself), while in all other gene trees, Dacryonaema and Unilacryma often formed a well-supported clade (BS = 57 \% for the nrDNA, $92 \%$ for RPB2, $75 \%$ for TEF-1 $\alpha$, and $80 \%$ for the mitochondrial dataset), as sister to a generally well-supported clade (99-100\% for the nrDNA, RPB2, and the mitochondrial dataset, unsupported for $T E F-1 \alpha$ ) containing the remaining 
Branch support:

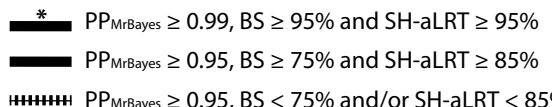

Dacrymyces stillatus anamorph UPS F-939814 Dacrymyces stillatus teleomorph UPS F-939814 Dacrymyces stillatus anamorph UPS F-939816 Dacrymyces stillatus teleomorph UPS F-939816 HнHн PP $_{\text {MrBayes }} \geq 0.95$, BS $<75 \%$ and/or SH-aLRT $<85 \%$ * Dacrymyces aff. stillatus TNS-F-15727 - Dacrymyces cf. minor TNS-F-15721 Guepiniopsis buccina AFTOL 888 Guepiniopsis buccina UPS F-940947 Guepiniopsis buccina TNS-F-15738

* Dacrymyces subantarcticensis HHB-18220 Dacrymyces subantarcticensis PDD 107988 * Dacrymyces pezizoides TNS-F-54909 Dacrymyces pezizoides TUMH 50290

Dacrymyces chrysospermus s.lat. AFTOL 528

* Dacrymyces chrysospermus s.lat. FPL 11353

Dacrymyces chrysospermus s.lat. TNS-F-15712

Dacrymyces aff. chrysospermus UPS F-593536

Dacrymyces dictyosporus HHB-8618

- Dacrymyces puniceus TNS-F-15711

- Dacrymyces subalpinus TNS-F-15730

* Cerinomyces ceraceus JGI

Cerinomyces ceraceus HHB-8969

Cerinomyces cf. grandinioides HHB-6908

Cerinomyces lagerheimii RLG-13487

- Dacryopinax spathularia TUB 019097

Dacryopinax spathularia TNS-F-21048

Dacryopinax spathularia AFTOL 454

Dacrymyces capitatus s.lat. CBS 293.82 cf. Ditiola sp. HHB-12010

Dis s.lat. TNS-15719

Dacrymyces stenosporus PDD 105018

Dacrymyces stenosporus PDD 107970
Unidentified Dacrymycetaceae 7 NBRC 110594

Unidentified Dacrymycetaceae 7 NBRC 110595

Unidentified Dacrymycetaceae 7 NBRC 110596

* Calocera pedicellata PDD 107830

Calocera pedicellata PDD 107925

Dacrymyces microsporus TNS-F-21050

Dacryopinax primogenitus JG

Dacryopinax indacocheae CRM-72

* Dacryoscyphus chrysochilus KUN F45014

Dacrymyces subarcticus TNS-F-21067

Unidentified Dacrymycetaceae 3 TNS-F-61322

- Dacryomitra pusilla UPS F-176774

Dacrymyces estonicus UPS F-940137

Dacrymyces estonicus UPS F-940138

- Unidentified Dacrymycetaceae 5 NBRC 110590

Ditiola radicata UPS F-939957

Ditiola radicata UPS F-939961

Dacrymyces parastenosporus PDD 104960

Dacrymyces parastenosporus PDD 104963

* Dacrymyces pachysporus PDD 105004 Dacrymyces pachysporus PDD 107916

* Dacrymyces longistipitatus PDD 107996

Dacrymyces longistipitatus PDD 107997

Calocera cornea AFTOL 438

Calocera cornea UPS F-940774

* Calocera cornea UPS F-940775

Calocera cornea CBS 124.84

Unidentified Dacrymycetaceae 1 NBRC 110568

Unidentified Dacrymycetaceae 1 TNS-F-61318

- Calocera cf. furcata JGI

Calocera viscosa s.lat. JGI

- Calocera viscosa s.lat. TNS-F-15704

Calocera viscosa s.lat. AFTOL 1679

Calocera viscosa s.lat. UPS F-940773

* * * $\quad$ * Unidentified Dacrymycetaceae 2 TNS-F-61311

Unidentified Dacrymyc

Unidentified Dacrymycetaceae 8 NBRC 110598

Unidentified Dacrymycetaceae 6 NBRC 110592

* Dacryopinax elegans TENN 066927

Dacryopinax elegans HHB-18731

Dacrymyces novae-zelandiae s.lat 2 TNS-F-21038

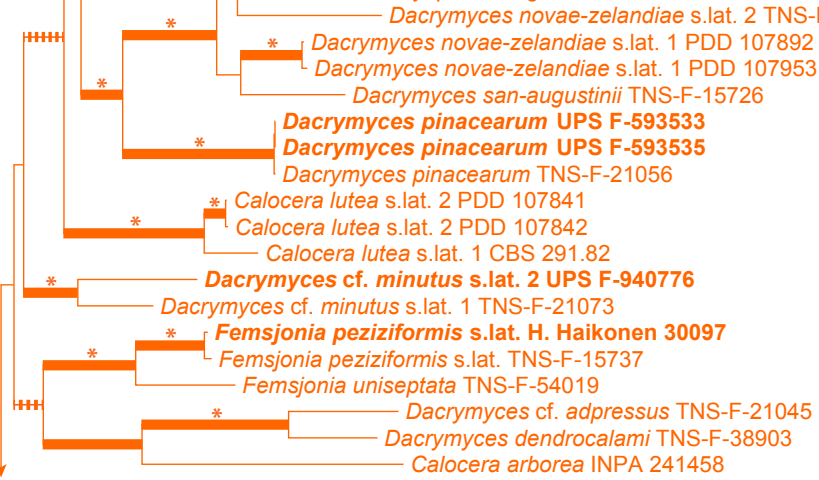

Fig. 8 Fifty percent majority-rule phylogenetic tree of the Dacrymycetes from the Bayesian analysis, based on the concatenated dataset. Numbers above branches indicate Bayesian posterior probability values $\left(\mathrm{PP}_{\mathrm{MrBayes}}\right.$ ), and numbers below branches indicate maximum likelihood bootstrap (BS) and ShimodairaHasegawa-like approximate likelihood ratio test (SH-aLRT) values. Branch support is indicated in the figure for branches above the species level. Samples with newly generated sequences are marked in bold. The scale bar represents the average number of substitutions per site. Clade $\mathrm{A}=$ Unilacrymaceae, clade $\mathrm{B}=$ Dacryonaemataceae, clade $\mathrm{C}=$ Cerinomycetaceae, clade $\mathrm{D}=$ Dacrymycetaceae. Subclades are explained in the text. 


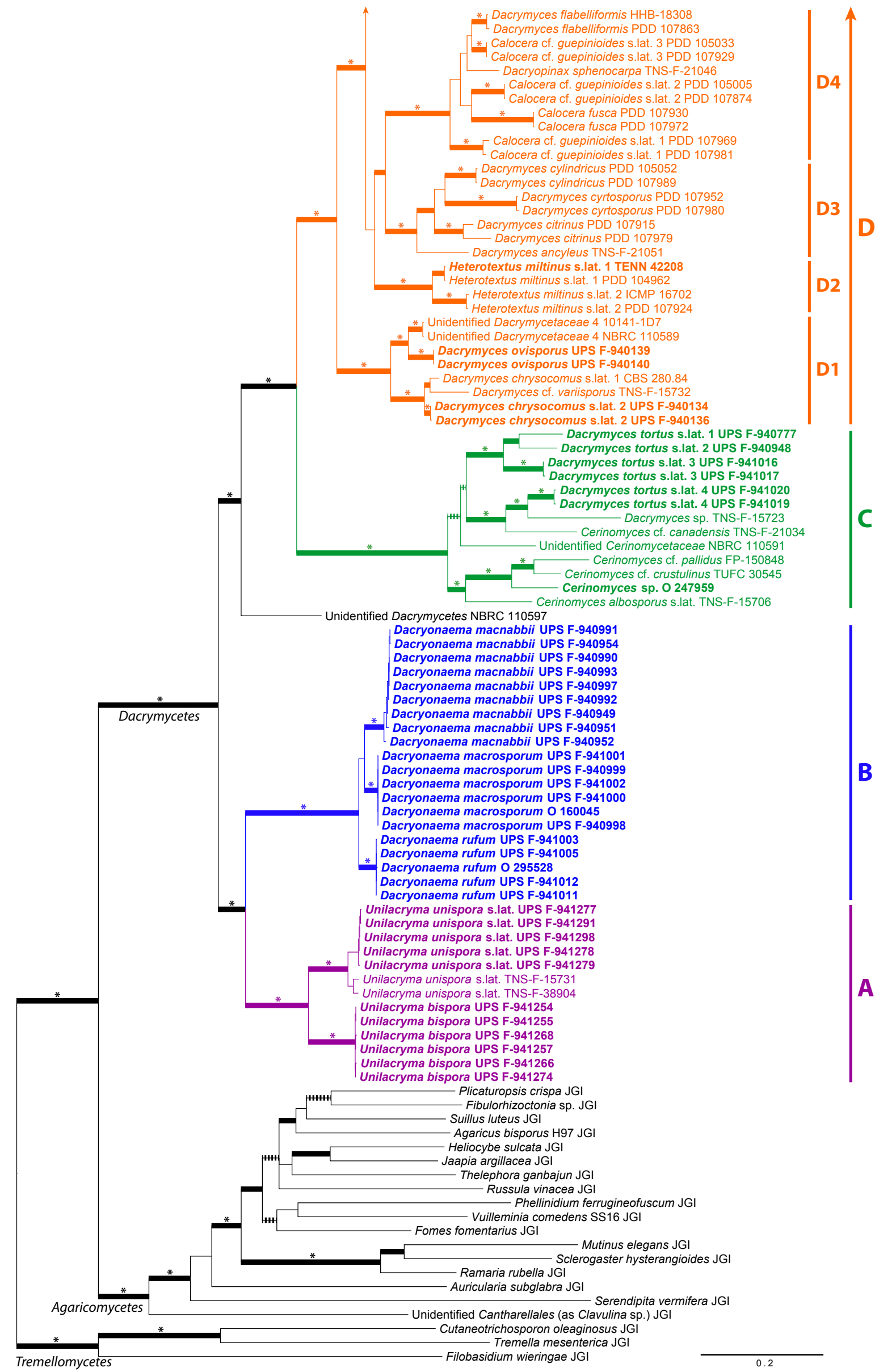

Fig. 8 (cont.) 


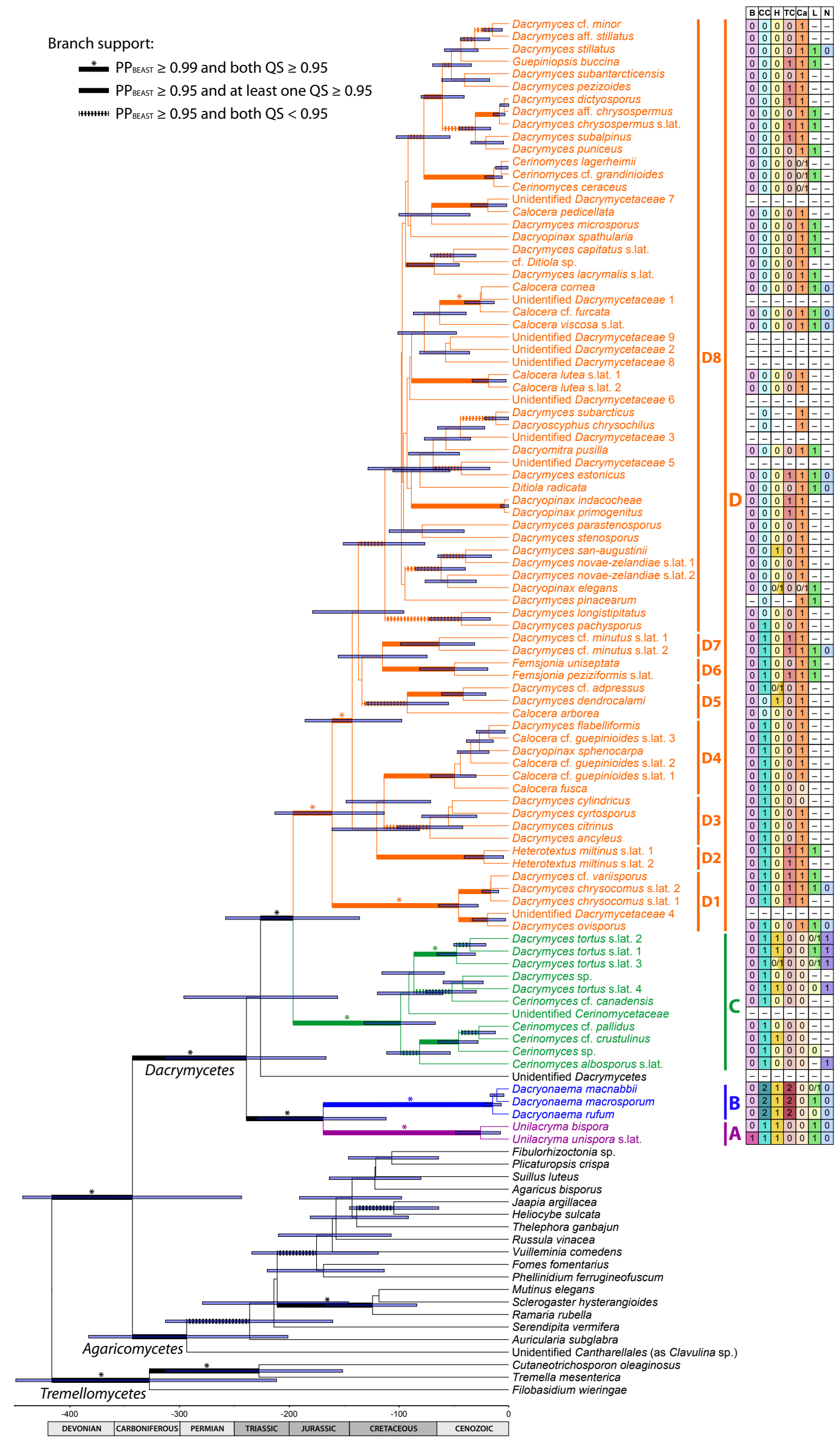

Fig. 9 Maximum clade credibility species tree chronogram from the coalescent Bayesian analysis. Node bars show the $95 \%$ HPD interval for the estimated heights of the nodes, and are provided only for branches with $\mathrm{PP}_{\mathrm{BEAST}} \geq 0.5$. Branch support is indicated in the figure. The grid bar represents the age in million years ago. Clade $\mathrm{A}=$ Unilacrymaceae, clade $\mathrm{B}=$ Dacryonaemataceae, clade $\mathrm{C}=$ Cerinomycetaceae, clade $\mathrm{D}=$ Dacrymycetaceae. Subclades are explained in the text. The character matrix with the states of the selected characters is provided to the right; $\mathrm{B}=$ basidia type, $\mathrm{CC}=$ clamp connections, $\mathrm{H}=$ hyphidia, $\mathrm{TC}=$ terminal cells of cortical $/$ marginal hyphae, $\mathrm{Ca}=$ carotenoids, $\mathrm{L}=$ cytoplasmic lipid content, $\mathrm{N}=$ nuclei; coded states are explained in Material and Methods; dashes indicate unknown states. 
Dacrymycetes (Fig. 7). We did not find any evidence of heterogeneous nucleotide composition, recombination, or heterotachy that could explain the topological differences. One possibility is that an RPB1 paralogue was sequenced in Dacryonaema. The RPB1 sequence of one sample of Dacryonaema macnabbii (UPS F-940952), amplified and sequenced twice, contained at its end substitutions that resulted in codon positions coding for unique amino acids, and a sequence from another sample (UPS F-940954) had a single base indel towards the end (both strands sequenced and no double signal noted), affecting the open reading frame. These problems were not noted in any of the other Dacrymycetes RPB1 sequences. Excluding suspicious parts of the alignment did not change the topological incongruence of the phylogeny. We consider it unlikely that the incongruence was caused by incomplete lineage sorting, since it affected very deep and comparatively long branches in the backbone, while relationships among closely related species were congruent. No other cases of incongruence were detected.

The best partitioning scheme and models for each partition in the final concatenated $\mathrm{ML}$ analysis were: (i) $12 \mathrm{~S}+\operatorname{ATP} 61^{\text {st }}$, GTR + I + Г4, (ii) ATP6 $2^{\text {nd }}$, GTR $+\Gamma 4$, (iii) $A T P 63^{\text {rd }}$, TIM $+\Gamma 4$, (iv) $18 \mathrm{~S}$, TIMe + I + Г4, (v) ITS1, TIM2 + I + Г4, (vi) ITS2, TPM2 $+\mathrm{F}+\mathrm{I}+\Gamma 4$, (vii) $5.8 \mathrm{~S}+28 \mathrm{~S}$, TN93 + I $+\Gamma 4$, (viii) $R P B 11^{\mathrm{st}}+$ $R P B 21^{\text {st }}, \mathrm{GTR}+\mathrm{I}+\Gamma 4$, (ix) $R P B 12^{\text {nd }}+R P B 22^{\text {nd }}, \mathrm{GTR}+1+\Gamma 4$, (x) RPB1 $3^{\text {rd }}+R P B 23^{\text {rd }}, \mathrm{GTR}+\mathrm{I}+\Gamma 4$, (xi) TEF-1a $1^{\text {st }}, \mathrm{GTR}+\mathrm{I}$ $+\Gamma 4$, (xii) TEF-1 $\alpha 2^{\text {nd }}$, TVM $+1+\Gamma 4$, and (xiii) TEF-1 $\alpha 3^{\text {rd }}$, GTR $+\lceil 4$. The two ML tree replicates had a similar topology, with InL $=-157377.647$ for the best one. The concatenated Bayesian analysis finished after $1.8 \times 10^{7}$ generations (ASDF $<0.01$ ); all parameters had a post-burn-in ESS $>1000$, all PSRFs $=1.000$ \pm 0.007 (most of them $1.000 \pm 0.002$ ), and RWTY plots did not show convergence problems with the tree topologies. The topology of the $50 \%$ majority-rule consensus tree was similar to that of the ML trees, and thus only the Bayesian tree with $\mathrm{PP}, \mathrm{BS}$ and $\mathrm{SH}$-aLRT values is shown in Fig. 8.

The final normalised quartet score in ASTRAL III was 0.983 in the five-locus analysis and 0.955 in the nine-locus analysis, indicating very low discordance among the single-locus trees. The trees computed from each of those two analyses were nearly identical. Only three runs reached convergence in the StarBEAST 2 analysis. Nearly every parameter had an ESS $>200$ (often $>1000$ ), including species tree parameters (posterior, likelihood, tree height, tree length), except the cySpeciationRate, which did not fully converge $(100<$ ESS $<200$, but see Discussion). RWTY plots did not show convergence problems among the species tree topologies. The ASTRAL III and StarBEAST 2 species trees were very similar, with minor differences only in unsupported groups. Thus, only the species tree from StarBEAST 2 with QS values from ASTRAL and PP values from StarBEAST analyses is shown in Fig. 9.

In all analyses, the Tremellomycetes, Agaricomycetes and Dacrymycetes were resolved as monophyletic groups and generally received high support $(\mathrm{BS}=100 \%, \mathrm{SH}-\mathrm{aLRT}=100 \%$, $\mathrm{PP}_{\text {MrBayes }}=1.00, \mathrm{QS}_{\text {ASTRAL5 }}=0.99, \mathrm{QS}_{\text {ASTRAL9 }}=0.99-1.00$, $\mathrm{PP}_{\mathrm{BEAST}}=1.00$, except the Agaricomycetes in the ASTRAL III analysis assuming five loci, with $\mathrm{QS}_{\text {ASTRAL5 }}=0.69$ ). The Dacrymycetes and the Agaricomycetes are sister groups and form a clade with high support (BS $=100 \%, \mathrm{SH}-\mathrm{aLRT}=100 \%, \mathrm{PP}_{\text {MrBayes }}$ $\left.=1.00, \mathrm{QS}_{\text {ASTRAL5 }}=0.99, \mathrm{QS}_{\mathrm{ASTRAL9}}=1.00, \mathrm{PP}_{\mathrm{BEAST}}=1.00\right)$. Within Dacrymycetes, we distinguish five main groups: (i) Unilacrymaceae (clade A), comprising Unilacryma unispora s.lat. and an unnamed Unilacryma with bisporic basidia;(ii) genus Dacryonaema (clade B), with three species; (iii) an isolated sample from the culture NBRC 110597; (iv) Cerinomycetaceae (clade C, several species); and (v) Dacrymycetaceae (clade D), including several genera and the majority of species. Support values and stem ages for the mentioned clades are summarised in Table 5. 


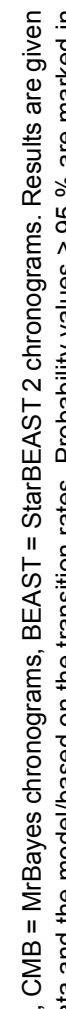

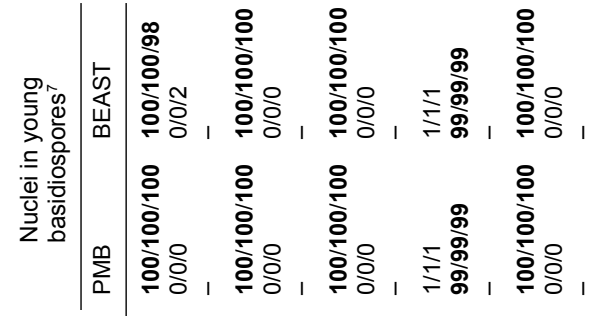

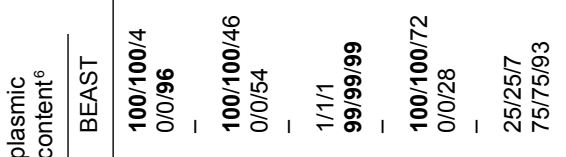
응.을

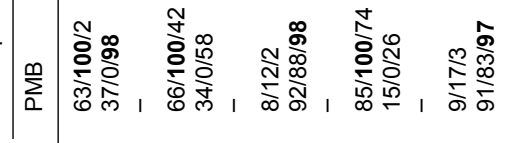

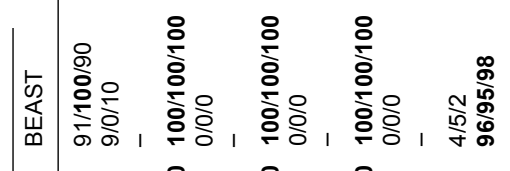

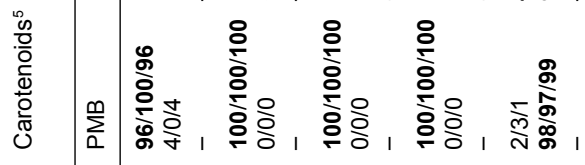
co 든, 잉

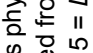
类券 䍗 空 的 क्षे 들

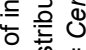

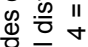

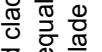
要

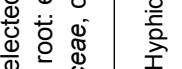
व की ते 贾 तٓ

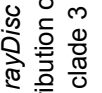
$\stackrel{0}{ \pm} \stackrel{2}{5}$

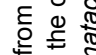

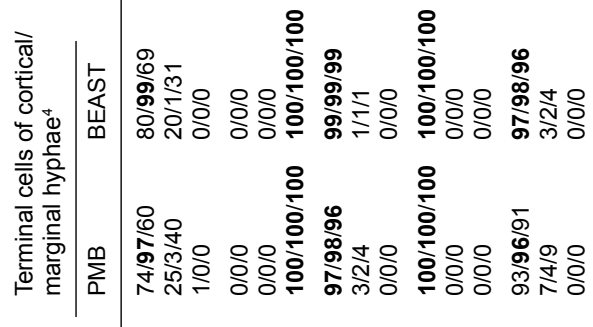
留

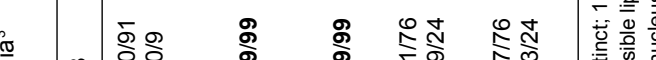

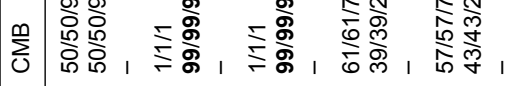

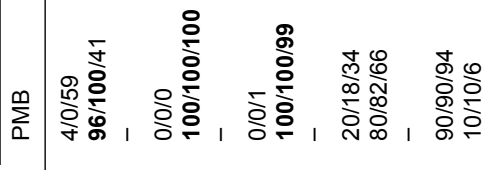

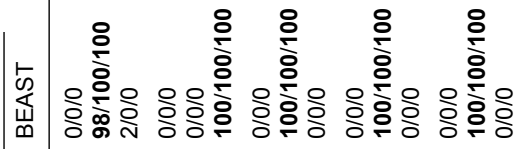
ब) ญे है 产

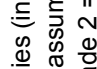

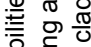

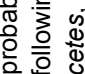
㐘 这

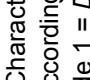

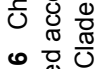

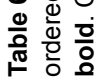

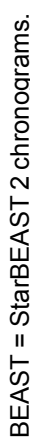

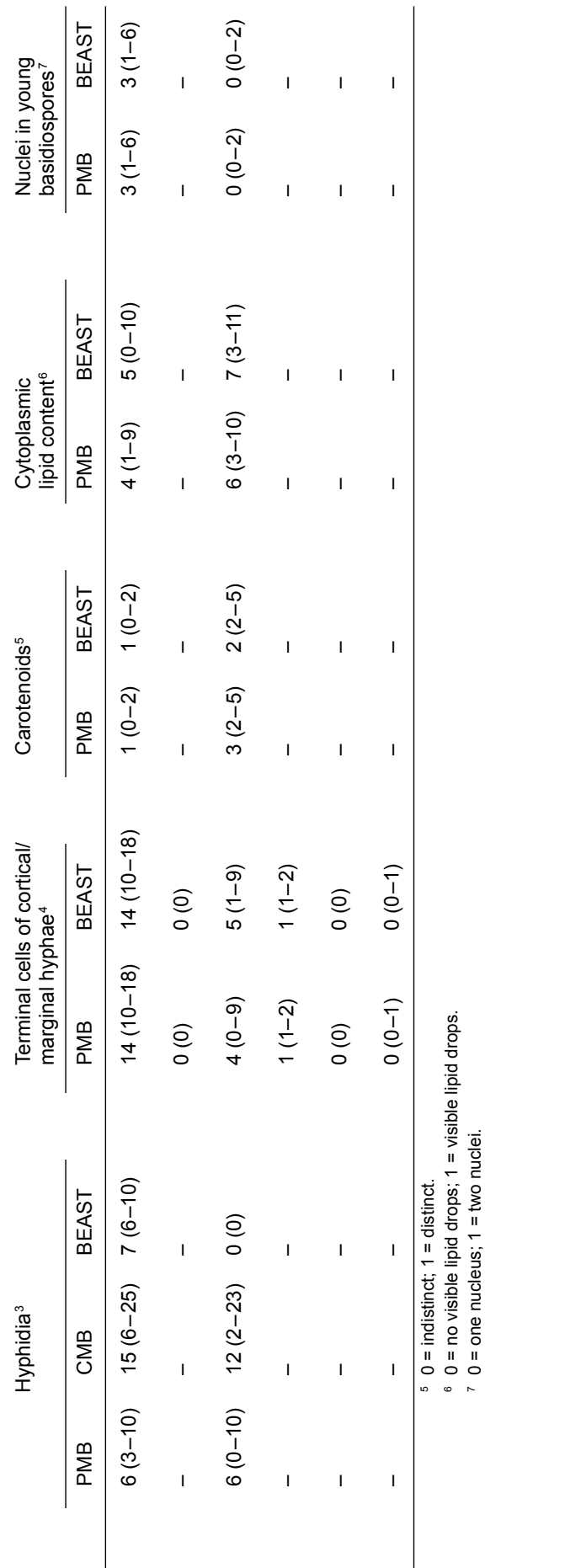

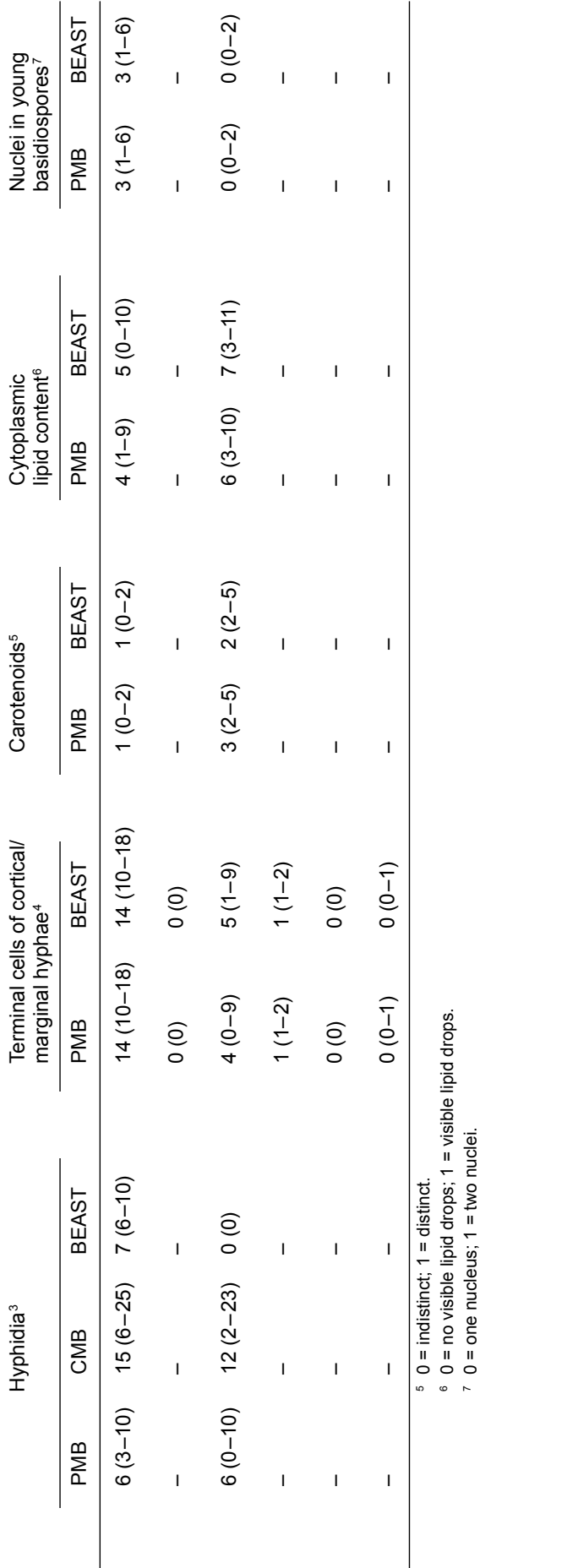

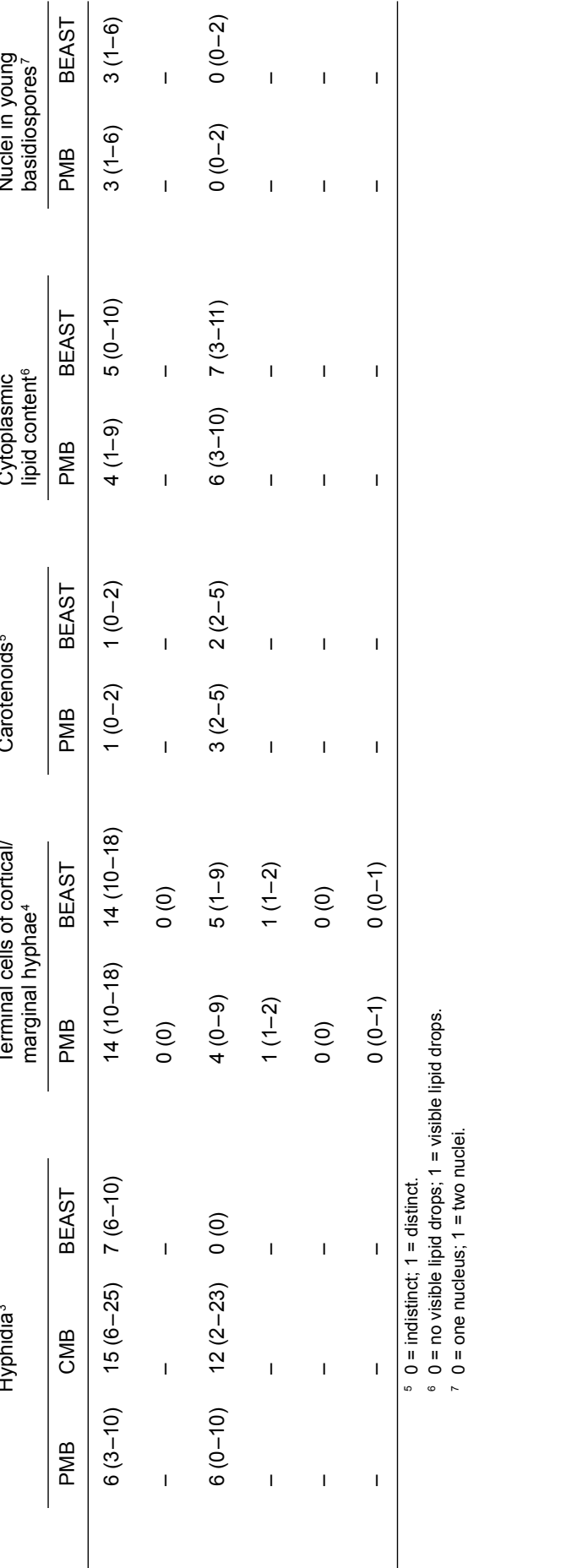

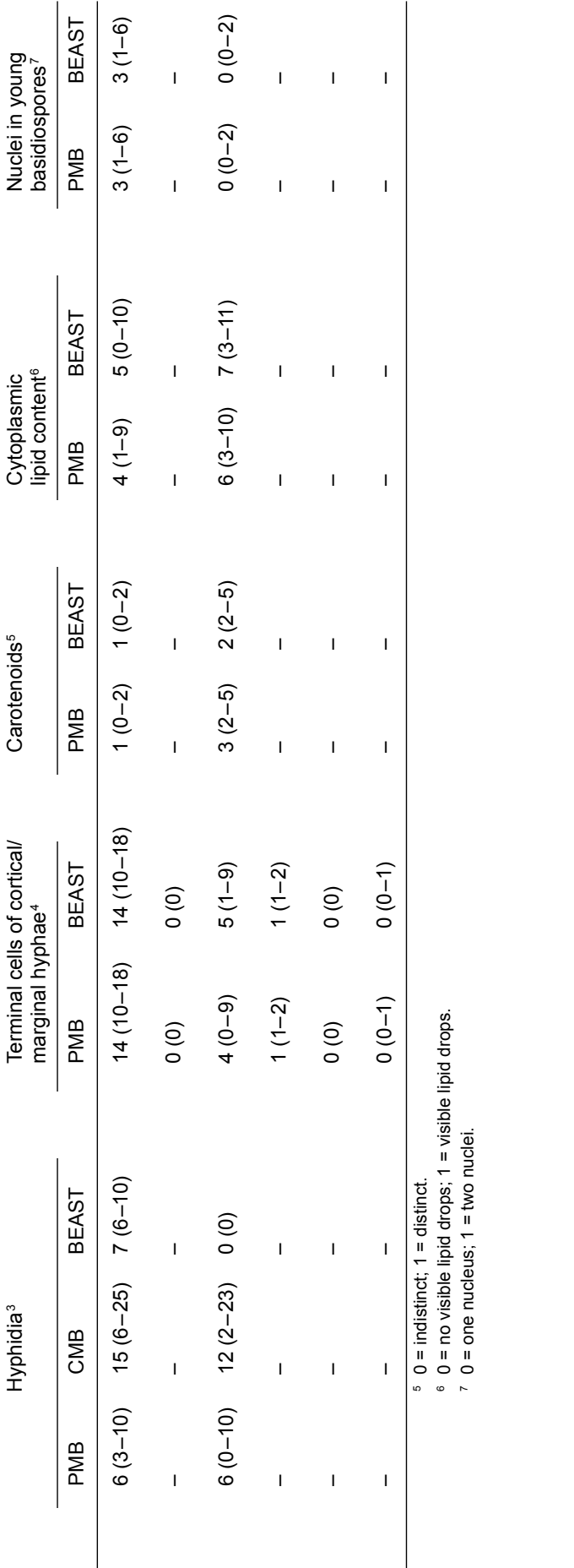

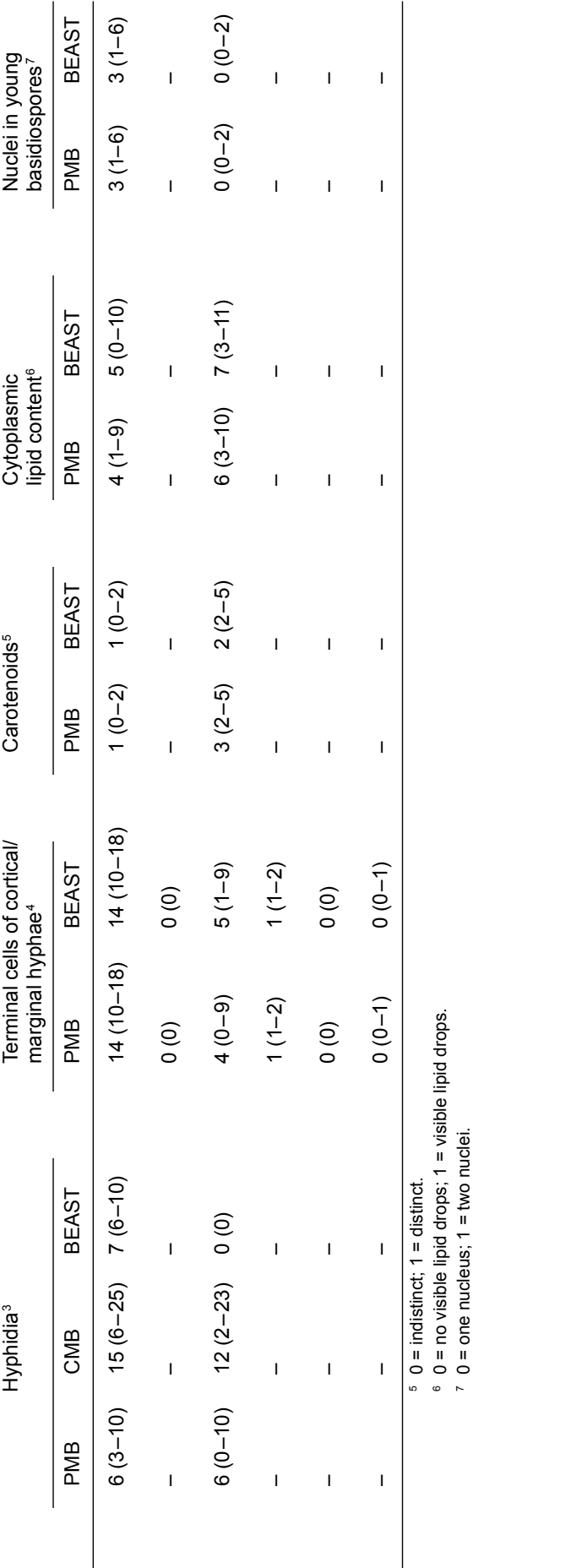

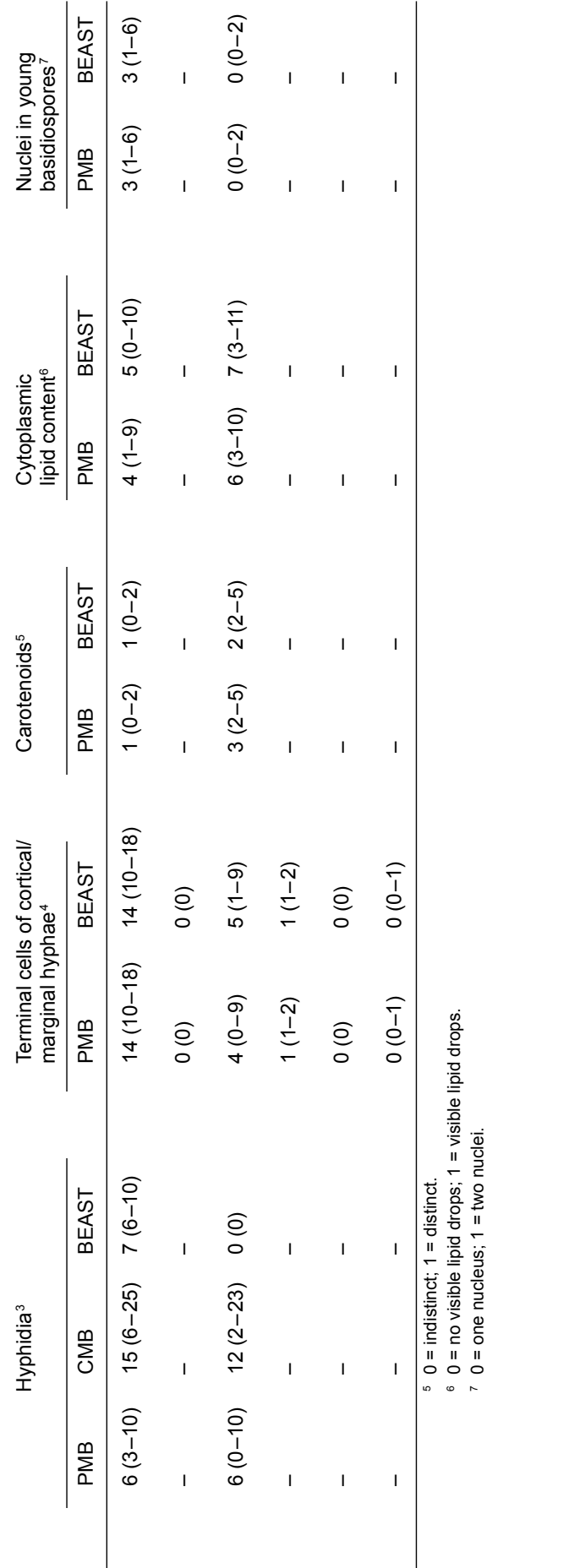

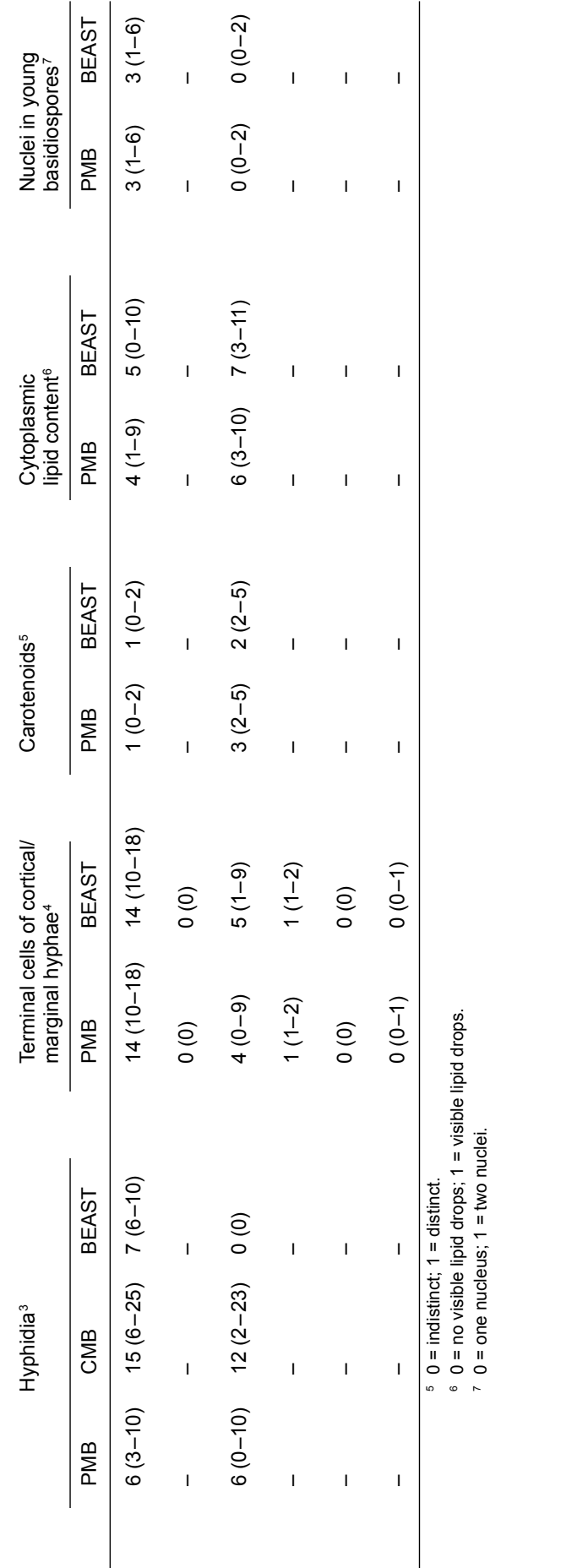

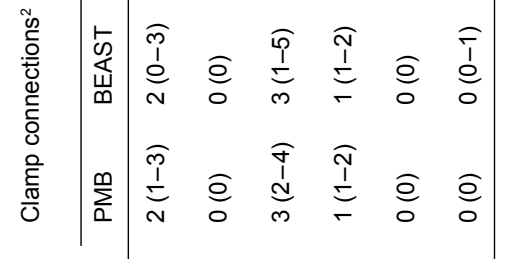

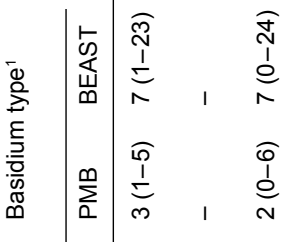

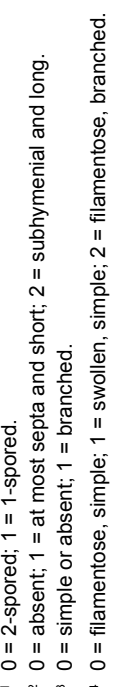


Dacryonaema and Unilacrymaceae are sister groups forming a clade with high support (BS $=100 \%$, SH-aLRT = $99 \%, \mathrm{PP}_{\mathrm{N}}$ $\left.=1.00, \mathrm{QS}_{\text {ASTRAL5 }}=0.98, \mathrm{QS}_{\text {ASTRAL9 }}=0.99, \mathrm{PP}_{\mathrm{BEAST}}=1.00\right)$, and so are Cerinomycetaceae and Dacrymycetaceae in most analyses $\left(\mathrm{BS}=100 \%\right.$, SH-aLRT $=100 \%, \mathrm{PP}_{\text {MrBayes }}=1.00, \mathrm{QS}_{\text {ASTRAL5 }}$ $\left.=0.67, \mathrm{QS}_{\mathrm{ASTRAL9}}=0.99, \mathrm{PP}_{\mathrm{BEAST}}=1.00\right)$. The clade formed by the sample NBRC 110957 + Cerinomycetaceae + Dacrymycetaceae is highly supported in the analyses using the concatenated dataset but not in two of the coalescence-based analyses $\left(\mathrm{BS}=99 \%, \mathrm{SH}-\mathrm{aLRT}=96 \%, \mathrm{PP}_{\text {MrBayes }}=1.00, \mathrm{QS}_{\text {ASTRAL5 }}\right.$ $=0.67, \mathrm{QS}_{\text {ASTRAL9 }}=0.95, \mathrm{PP}_{\mathrm{BEAST}}=0.86$ ). The nodes in the Dacrymycetes backbone, uniting groups $\mathrm{i}-\mathrm{v}$, are consistent among all analyses (Fig. 8-9).

Several internal subclades can be identified in the Dacrymycetaceae, and we highlight eight of them with taxonomic or nomenclatural relevance: D1-D8. All are well-supported except D8 that received high support only from SH-aLRT (100\%), $\mathrm{PP}_{\text {MrBayes }}$ (1.00), and $\mathrm{PP}_{\mathrm{BEAST}}(1.00)$, and D3 and D5 that received high support in all analyses except ASTRAL III. For details on the included species and morphological characterization of these subclades, see Discussion.

\section{Character evolution}

Posterior probabilities of character states (pp) from the SIMMAP simulations at selected nodes were similar between the MrBayes phylograms and the StarBEAST 2 chronograms, except for the hyphidia (Table 5). The ancestor of the Dacrymycetes was inferred to have simple hyphidia with $67 \%$ pp in the analysis using MrBayes phylograms, $87 \%$ pp based on MrBayes chronograms, and $97 \%$ pp when using Star BEAST 2 chronograms. The ancestor of Cerinomycetaceae was inferred to have branched hyphidia based on MrBayes phylograms (65\% pp), while using chronograms from MrBayes and StarBEAST 2 simple hyphidia was the most probable state, with $83 \%$ and $98 \%$ pp, respectively.

The ancestor of the Dacrymycetes was inferred to have 2-spored basidia, hyphae with short clamp connections at most septa, not visible carotenoids, conspicuous cytoplasmic lipid contents, and uninucleate young basidiospores (84-100\% pp). The ancestor of Dacryonaemataceae had 2-spored basidia, hyphae with long clamp connections mostly at the subhymenium and the bases of basidia and hyphidia, branched hyphidia, cortical/marginal hyphae with branched, and undilated terminal cells, no visible carotenoids, and uninucleate young basidiospores (83-100\% pp). The ancestor of Unilacrymaceae had 2-spored basidia, hyphae with short clamp connections at most septa, branched hyphidia, simple and undilated terminal cells of cortical/marginal hyphae, invisible carotenoids, conspicuous cytoplasmic contents, and uninucleate young basidiospores $(81-100 \% \mathrm{pp})$. The ancestor of Cerinomycetaceae had 2-spored basidia, hyphae with short clamp connections at the majority of septa, cortical/marginal hyphae with simple and undilated terminal cells, invisible carotenoids, and binucleate young basidiospores (99-100 \% pp). Finally, the ancestor of Dacrymycetaceae had 2-spored basidia, hyphae with short clamp connections at most septa, hyphidia simple or absent, cortical/marginal hyphae with simple and undilated terminal cells, visible carotenoids, conspicuous cytoplasmic lipid contents, and uninucleate young basidiospores (86-100\% pp).

RayDISC results (Table 6 ) largely agreed with those from SIMMAP, including the different inferences for the hyphidia when a different tree was used. However, state reconstructions for the basidium type and the amount of lipid content varied considerably at the root node (ancestor of the Dacrymycetes) depending on the assumed distribution of states at that node. A 1-spored basidium was inferred as the ancestral state with $100 \%$ pp assuming an equal distribution of states in MrBayes phylograms, while a 2-spored basidium was inferred with $100 \%$ $\mathrm{pp}$ both when the distribution of states is calculated using the estimated transition rates (both trees) and when it is estimated from the data and the model in MrBayes phylograms. The amount of cytoplasmic lipid content is inferred as high when the distribution of states is calculated using the estimated transition rates (96-98\% pp), while it is inferred as low when it is estimated from the data and the model (100\% pp in both trees) and also assuming an equal distribution of states for the BEAST chronograms (100\% pp).

The median transformation counts (Table 7) show that the 1-spored basidium seems to be a rarely acquired apomorphic state, probably as a reduction of the 2 -spored basidium, although there is a substantial uncertainty in the counts from the chronograms. Clamp connections were lost only in a couple of internal groups in the Dacrymycetaceae (remarkably in subclade D8, Fig. 9), and reversions to the state with clamp connections were uncommon. The hyphidium branching seems to have increased in most clades, although the acquisition of branched hyphidia was rare in Dacrymycetaceae. The terminal cells of cortical/ marginal hyphae showed some flexibility, as the transformation counts show numerous transitions from simple and not swollen cells into more or less conspicuously dilated cells in the Dacrymycetaceae. Some reversions to the ancestral state were also inferred. The branched and not swollen cells most probably appeared only once (in the Dacryonaemataceae). The carotenoids were most likely acquired only once, by the ancestor of the Dacrymycetaceae, with a few secondary losses. The visible lipid content of the cytoplasm in basidia and basidiospores was reduced a few times (e.g., Dacryonaema rufum and some species in the Cerinomycetaceae), although increases in visibility also occurred. The binucleate young basidiospore state is an acquired state in the Cerinomycetaceae.

\section{Dacryonaema and Unilacryma phylogeny and} species delimitation analyses

No incongruence among loci were detected. Both ML and Bayesian analyses based on the concatenated dataset resulted in trees with a similar topology. The best partitioning scheme and models for each partition in the ML analysis were: (i) $18 \mathrm{~S}+5.8 \mathrm{~S}$ + TEF-1 $\alpha 2^{\text {nd }}, \mathrm{K} 2 \mathrm{P}+\mathrm{I}$; (ii) ITS1 + RPB1 (Dacryonaema) $1^{\text {st }}+$ $R P B 1$ (Unilacryma) $1^{\text {st }}+R P B 21^{\text {st }}$, TIM2 + I; (iii) ITS2, K2P; (iv) $28 \mathrm{~S}+\mathrm{TEF}-1 \alpha 1^{\text {st }}, \mathrm{TN} 93+\mathrm{I}$; (v) RPB1 (Dacryonaema) $2^{\text {nd }}$ $+R P B 1$ (Unilacryma) $2^{\text {nd }}+R P B 22^{\text {nd }}, \mathrm{TPM} 3+\mathrm{F}$; (vi) $R P B 1$ (Dacryonaema) $3^{\text {rd }}+R P B 1$ (Unilacryma) $3^{\text {rd }}+R P B 23^{\text {rd }}, H K Y$ + Г4; (vii) TEF-1a $3^{\text {rd }}$, TPM2 + F + Г4; (viii) ATP6 first codon position ( $\left.1^{\text {st }}\right)$, TN93 + I; (ix) ATP6 second codon position $\left(2^{\text {nd }}\right)$, TPM3u + F + I; (x) ATP6 third codon position ( $3^{\text {rd }}$ ), TIM3; and (xi) indels, MK + ASC. Both ML replicates yielded trees with a nearly identical topology (minor changes in a few unsupported branches within species), with $\operatorname{lnL}_{1}=-18219.068$ and $\mathrm{InL}_{2}=-18219.016$ (best).

The MrBayes analysis was automatically halted after $8 \times 10^{6}$ generations, when the ASDSF dropped below 0.01. At this point, all ESSs after discarding the burn-in were $>1000$, and all tree and model parameters had PSRFs $=1.000 \pm 0.001$.

The STACEY analysis yielded a chronogram with a similar topology to the phylograms obtained using the concatenated dataset. Thus, only the STACEY chronogram with support values from all analyses is shown in Fig. 10. According to SpeciesDelimitationAnalyzer, the most probable delimitation involves six species (40.7\% posterior probability). All other delimitations had a posterior probability of $<5 \%$.

Dacryonaemataceae and Unilacrymaceae were resolved as monophyletic, highly supported groups. Within the Dacryonaemataceae, three fully or nearly fully supported main clades are resolved $\left(\mathrm{BS}=100 \%, \mathrm{SH}-\mathrm{LLRT}=99-100 \%, \mathrm{PP}_{\text {MrBayes }}=1.00\right.$, 


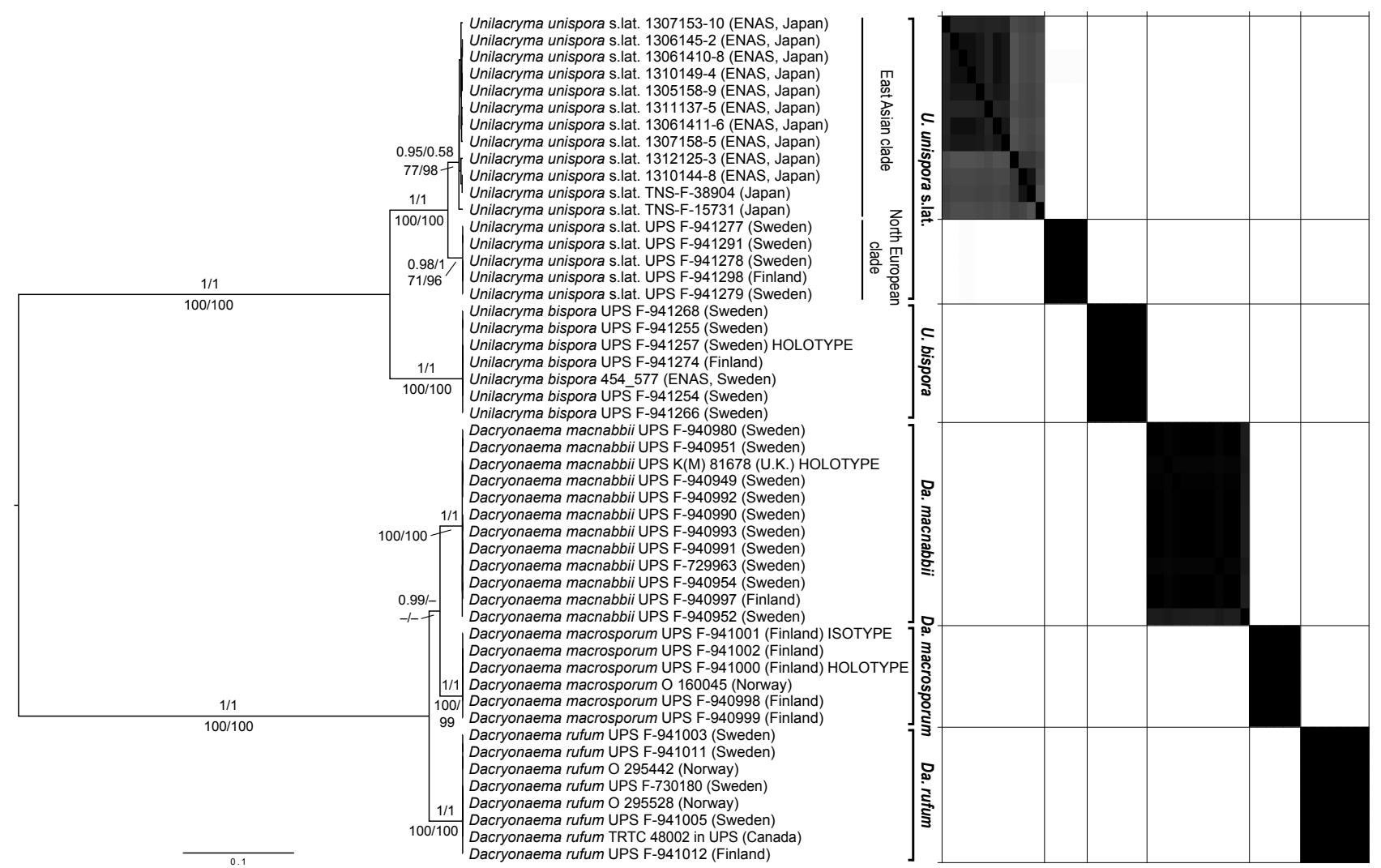

Fig. 10 STACEY species delimitation analysis with chronogram and similarity matrix for the Dacryonaema + Unilacryma dataset. Numbers above branches indicate Bayesian posterior probability $\left(\mathrm{PP}_{\mathrm{STACEY}} / \mathrm{PP}_{\mathrm{MrBayes}}\right)$ values, and numbers below branches indicate maximum likelihood bootstrap (BS) and ShimodairaHasegawa-like approximate likelihood ratio test ( $\mathrm{SH}$-aLRT) values.

$\mathrm{PP}_{\text {STACEY }}=1.00$, and the same clades are inferred as putative species (Da. macnabbii, Da. macrosporum, and Da. rufum) in the STACEY species delimitation analysis. The posterior probability among specimens to belong to the same inferred group was high according to the similarity matrix ( $p p>0.88)$. The posterior probability that any of the samples in one of the three putative species may belong to any of the other two putative species was zero.

Within Unilacrymaceae, two fully supported clades were resolved, representing $U$. bispora and $U$. unispora s.lat. The posterior probability of all specimens of $U$. bispora belonging to the same cluster inferred by STACEY was high ( $p p>0.99$ ), and the probability that any of the samples belong to any of the $U$. unispora s.lat. groups was zero. The clade formed by $U$. unispora s.lat. samples is further divided into two subclades, one including all North European samples (BS $=71 \%$, SH-aLRT $\left.=96 \%, \mathrm{PP}_{\text {MrBayes }}=1.00, \mathrm{PP}_{\text {STACEY }}=0.98\right)$ and the other including all East Asian samples (BS $=77 \%$, SH-aLRT $=98 \%$, $\left.\mathrm{PP}_{\text {MrBayes }}=0.58, \mathrm{PP}_{\text {STACEY }}=0.95\right)$. The STACEY analysis inferred these subclades as two putative species. Within the North European group, all specimens had high posterior probability of belonging to the same group ( $p p>0.99$ ). Within the East Asian group, the posterior probability of each sample belonging to the same group was highly variable $(0.67<p p<0.94)$. The posterior probability of North European and Japanese samples belonging to the same cluster was low ( $p p<0.02)$.

\section{DISCUSSION}

\section{Phenotypic characters}

\section{Cytology as a source of taxonomic characters}

Vacuolar cell contents have been rarely noted in Dacrymycetes by other researchers, and there are scarce records of them in the literature. For instance, they were probably drawn by Nannfeldt (1947) for some basidiospores of Dacryonaema rufum (e.g., his f. 2 showing vacuolated ungerminated basidiospores after 5 days in water). Also, the photograph for explaining the ontogeny of basidia in Dacrymyces stillatus included by Oberwinkler (2014: f. 3 of that study) showed conspicuous VBs in some basidia. Unfortunately, we did not see any taxonomically relevant variation of the VBs in the Dacrymycetes.

The amount of lipid contents, routinely used as a taxonomic character in several groups of Ascomycota (Baral 1992), has been poorly explored in the Basidiomycota. The higher lipid content in the cytoplasm of Dacrymycetaceae and Unilacrymaceae compared to Cerinomycetaceae seems useful for identifying the latter family, although $D$. tortus s.lat. 1 is a clear exception in which conspicuous lipid drops are easily observed. This species might be confused with others in the Dacrymycetaceae, especially as taxa in the $D$. tortus complex also have dacrymycetoid fruitbodies. They rarely coalesce and may have obvious orange tinges when young. The basidia are sometimes comparatively long, in which case their dimensions and shape overlap with those of some Dacrymycetaceae.

There are not many studies focused on the nuclear stages in the Dacrymycetes. The first mentions appeared in Dangeard (1895), Istvanffi (1895), Juel (1898), and Maire (1902), who noted that a single nucleus passes into each young basidiospore when attached to the basidium. Bodman (1938) carefully explained the karyological states of basidia and basidiospores of Dacryopinax spathularia, and suggested that of the four postmeiotic nuclei, two migrate to each basidiospore (thus, basidiospores are haploid and uninucleate) and two degenerate in the basidium. Lange (1955), for the Dacrymycetaceae (as 'Dacryomycetace$\left.a e^{\prime}\right)$, suggested an ontogeny of the basidia in agreement with Bodman (1938), and depicted dikaryotic vegetative cells and 'oidia-like' mitospores, that might correspond to a particular interpretation of the arthrosporic state of, e.g., D. stillatus. 
Oberwinkler (2014) described nuclear stages in agreement with Bodman (1938) and Lange (1955) when detailing the general life cycle of Dacrymycetes, exemplified by Dacrymyces stillatus. Duncan \& Chisholm (1982) discovered a third nuclear division (pattern B of Duncan \& Galbraith 1972) in the basidia of Dacrymyces stillatus, but basidiospores were still uninucleate (six nuclei remaining in the old basidium). McLaughlin et al. (2016) studied the ontogeny and cytology of a newly described species, Dacryopinax primogenitus, and observed the dikaryotic nature of the vegetative cells and conidia, as well as a similar pattern of post-meiotic nuclear division noted by Duncan \& Chisholm (1982).

Most of this information closely agrees with our observations in Dacrymycetaceae. Although the observation of some nuclear stages was possible without stains, a clear observation of the nuclei often needs specific staining. Most nuclear staining techniques involve a long process (sometimes days) that requires the fixation of the samples prior to staining, and uses some chemicals unusual in rutine taxonomic studies (Bodman 1938). As an alternative, we tried a fast $(<1 \mathrm{~min})$ staining with alkaline toluidine blue, not requiring previous fixation of the samples (see Material and Methods). This gave reasonably good results, allowing us to confirm several of our observations, despite the fact that only a low number of cells were often properly stained. However, we have not been able to observe the pattern $B$ of the third nuclear division reported by Duncan \& Chisholm (1982) in any species.

Our observations of the uninucleate state in basidiospores of Dacrymycetaceae agree with nearly all previous information about Dacrymycetaceae (see above), except for Vandendries (1938), who claimed that basidiospores in Calocera cornea are dikaryotic. Nannfeldt (1947) likely illustrated uninucleate basidiospores in Dacryonaema rufum when still attached to the basidium (f. 2 on p. 328 in that study), although it was not explicitly indicated whether or not the central, rounded organelle depicted was the nucleus. Although further research is needed to confirm the number of nuclei in Unilacrymaceae basidiospores, young (= non-septate) basidiospores in both species seem to be uninucleate (e.g., Fig. 4t). The ancestral state reconstruction infers the uninucleate state of the basidiospores as the most probable one for the ancestors of the Dacrymycetes and also of Dacrymycetaceae, Unilacrymaceae, and the genus Dacryonaema (Table 5-6, Fig. 9).

Unlike the other families, binucleate young basidiospores seem to be the rule in the Cerinomycetaceae. Maekawa (1987) already indicated that recently discharged basidiospores of Ce. aculeatus were mostly binucleate. In addition to this species, we have consistently observed binucleate young basidiospores in five out of six species of Cerinomycetaceae that were studied based on living specimens (three of them included in the present phylogenetic analyses). Binucleate basidiospores in the Basidiomycota are not uncommon in some taxonomic groups, as noted by Duncan \& Galbraith (1972). The binucleate condition is typically a product of postmeiotic nuclear division pattern D, i.e., the original haploid post-meiotic nucleus in the young basidiospore further divides inside the basidiospore and both daughter nuclei remain. Dacrymyces tortus s.lat. 1, in which observations of nuclei are difficult because of high amounts of lipid bodies in the basidiospore cytoplasm, showed both uninucleate and binucleate basidiospores when young and aseptate. This would still agree with pattern $D$, if we consider the third nuclear division more delayed than in other species, and then very recently discharged basidiospores may be uninucleate for a short period of time. Should further studies on a wider number of species confirm our observations, a comparatively long binucleate state of the basidiospores (i.e., not starting only immediately prior to septation) may represent a new synapo- morphy and distinguishing character for the Cerinomycetaceae. With the present data, ancestral state reconstruction analyses indeed infer the binucleate state of the basidiospores as plesiomorphic but unique to the Cerinomycetaceae.

\section{Lineage-specific presence of carotenoids}

It is generally accepted that the yellow/orange colours in the Dacrymycetes are caused by the presence of various carotenoid pigments (Gill \& Steglich 1987, Oberwinkler 2014). The precise composition has been investigated in Calocera, Dacrymyces, and Dacryopinax (e.g., Goodwin 1953, Hanna \& Bulat 1953, Fiasson 1973, Czeczuga 1980), and $\beta$-carotene has been found to be among the most abundant substances. Carotenoids are lipidophilous and thus typically found in lipid globules or in membranes (Echavarri-Erasun \& Johnson 2002). We clearly observed them only in the lipid bodies of the cytoplasm, as reported for many other fungi (e.g., Cederberg \& Neujahr 1970, Riley 1978, Griffin 1994). However, Vail \& Lilly (1968) reported the highest amount of carotenoids in the 'cell wall fraction' of Dacryopinax spathularia. The colour of fresh fruitbodies in the Dacrymycetes varies considerably, especially at the family level, and species outside the Dacrymycetaceae are commonly creamish, brownish, or greyish, instead of bright yellow or orange (Fig. 5a-d). Already Nannfeldt (1947) noted that neither the basidia nor the basidiospores of Dacryonaema rufum contain yellow lipid drops, in contrast with their common occurrence in many species of Dacrymycetaceae. Oberwinkler (2014) reported that some taxa, such as Ditiola haasii and Cerinomyces species were exceptions to the rule that the basidiocarp pigmentation is caused by yellowish to orange carotenoids in the Dacrymycetes. The spore print is often reported as yellow to orange, and this is the case in many species within the $D a-$ crymycetaceae (e.g., Calocera viscosa, Fig. 5e, Dacrymyces aureosporus, $D$. chrysospermus), but there are also reports of species with pale or whitish spore prints (e.g., Cerinomyces albosporus, Boidin \& Gilles 1986). Other published information may not be as accurate. For instance, some taxa with clearly yellowish spore prints have been incorrectly reported as having a whitish spore print (e.g., the generic descriptions of Dacrymyces, Ditiola, Femsjonia and Guepiniopsis in Læssøe \& Petersen 2014).

Our results indicate that there has been a sharp increase in the amount of carotenoids in the Dacrymycetaceae, whereas these pigments are not clearly observable, perhaps even absent, in Cerinomycetaceae, Unilacrymaceae, and in Dacryonaema. Also, the colour of the fruitbodies and that of colonies grown in pure culture seem to be related. Shirouzu et al. (2009) only reported yellow to orange colours for species in the Dacrymycetaceae (pale or whitish colonies also occurred), while the colonies in the clades corresponding to Cerinomycetaceae and Unilacrymaceae were constantly white.

Carotenoids have been demonstrated to play a role in the cell protection against oxidative stress and photoreactions (Will et al. 1984, Will III \& Scovel 1989, Moliné et al. 2010, Avalos \& Limón 2015). This function was demonstrated in pure cultures of Dacryopinax spathularia (Goldstrohm \& Lilly 1965). However, several of the species most frequently found on exposed wood, receiving large amounts of sun-light, possess small or no amounts of carotenoids, e.g., Dacryonaema, Unilacryma, and at least one Cerinomyces species (D. tortus s.lat. 3). Therefore, a putative ecological advantage of these pigments may also be related to other functions of carotenoids (e.g., as precursors of some active compounds, Avalos \& Limón 2015).

lodine solutions are known to react with the carotenoid contents in different groups of fungi, typically exemplified by the greening of the paraphyses of several ascomycetes with Lugol's or Melzer solutions (Malençon \& Bertault 1971, Schumacher 
1990, other coloured reactions also occur in this group). The carotenoid contents in the Dacrymycetaceae display a greenish to greenish grey reaction in Lugol's solution, while this reaction has not been observed in any species of Cerinomycetaceae, Dacryonaema, or Unilacryma. The combination of direct observation of the fruitbodies and cell cytoplasm, plus their greenish reaction with iodine, may allow researchers to quickly and objectively assess the presence and relative amount of carotenoids.

There are, however, some species in the Dacrymycetaceae with colours other than the common yellow or orange, such as the whitish or very pale coloured Calocera cavarae, Ca. pallidospathulata (Reid 1974), Ca. fusca, Ditiola haasii (Oberwinkler 1989), and Dacrymyces cylindricus (Shirouzu et al. 2017). For these taxa, it is reasonable to suggest a secondary loss of the pigmentation, since DNA sequence data have demonstrated that they belong to at least three different internal clades of the Dacrymycetaceae dominated by species with yellow-orange pigments (Shirouzu et al. 2013, 2017). This agrees with both the present phylogenies and our ancestral state reconstructions including $\mathrm{Ca}$. fusca and D. cylindricus (Fig. 9). The loss of carotenoids in certain species or specimens has already been reported in other fungi (e.g., Baral 2004 in Sarcoscypha, Hansen et al. 2013 in some clades of Pyronemataceae, Olariaga et al. 2015 in Cantharellus). In addition, fruitbodies of species that normally produce carotenoids often produce more pigment when exposed to sunlight. In contrast, when they grow in absence of light they are often paler or even whitish, a phenomenon well known in some other Basidiomycota (Pilz et al. 2003, Olariaga et al. 2015). The Dacrymycetes are not an exception. Such uncommon cases do not invalidate the usefulness of carotenoid content for taxonomic purposes. With the current data, any species with a conspicuous and high carotenoid content would be tentatively best placed in the Dacrymycetaceae, since the presence of distinct carotenoids is inferred as being acquired in the ancestor of the family, and few reversions to the plesiomorphic carotenoid-deficient condition occurred (Table 5-7). We recommend that future studies on Dacrymycetes include careful observations of fresh, unstained samples and the subsequent reporting of approximate carotenoid content.

\section{Other morphological characters}

There is only one previous study including ancestral reconstruction analyses of multiple morphological characters in the Dacrymycetes (Shirouzu et al. 2013). They proposed that the macromorphology of the fruitbodies is highly homoplastic and rarely a synapomorphy for any higher clade, and the same was true for the wall thickness of marginal hyphae and, to some extent, also basidiospore septation. By contrast, other characters, such as the presence or absence of clamp connections, turned out to characterise some phylogenetic lineages.

In agreement with Shirouzu et al. (2013), our phylogeny shows that clamped septa seem to be the plesiomorphic state in the Dacrymycetes, while simple septa appear in some derived clades only within the Dacrymycetaceae. In addition, we found that clamp connection morphology and location may be informative for high-level taxa delimitation. Loop-like, long and angulose clamp connections (i.e., long medallions), almost restricted to subhymenial hyphae and the bases of basidia and hyphidia, are characteristic of the three known species of Dacryonaema. This specific morphology and distribution do not consistently appear in any other group, as was inadvertently indicated by Reid (1974) when he commented on the uniqueness of the Da. macnabbii (as Dacrymyces macnabbii) clamps. Loop-like, but normally shorter and more rounded clamp connections are present in some Dacrymycetes, as noted by, e.g., Martin (1949). We have observed them in some species, for instance in the
Dacrymyces tortus complex and in Unilacryma, and infrequent transitions to Dacryonaema-like, longer clamp connections may occur. It is interesting to note that Unilacrymaceae, the sister group to Dacryonaema, also lacks clamp connections at some septa, but this feature may occur in some species of Cerinomycetaceae and Dacrymycetaceae with typically clamped septa, due to the formation of secondary simple septa.

The morphology of cortical/marginal hyphae was used as a key character by Oberwinkler $(1994,2014)$ to circumscribe some genera, and it is commonly found in the literature as important to characterise, e.g., Guepiniopsis and Heterotextus (McNabb $1965 c-d)$. Our ancestral state reconstruction analyses show that the presence of branched and anastomosing terminal cells with walls that become gelatinised in $\mathrm{KOH}$ is plesiomorphic and synapomorphic for Dacryonaema but absent in other clades, where terminal cells of cortical/marginal hyphae tend to be simple and anastomoses rare. Indeed, the ancestral condition predicted for the Dacrymycetes, Cerinomycetaceae, Dacrymycetaceae, and Unilacrymaceae is the presence of simple and not swollen terminal cells, although some uncertainty exists depending on the assumptions made at the root node (Table 5-6). These terminal cells are conspicuously dilated and morphologically diverse in some groups (e.g., Femsjonia, Heterotextus), suggesting that this character should be carefully addressed in future studies, particularly in the Dacrymycetaceae.

The type of basidium, i.e., forked or simple, cannot any longer be considered a good character to distinguish Unilacryma from other clades, as Unilacryma bispora constantly has bisterigmate basidia. Simple, 1-spored basidia are highly unusual in the Basidiomycota, and in most cases species characterised by 1 -spored basidia belong to genera with a variable number of spores produced per basidium, e.g., Heteroacanthella, Lactarius, Oliveonia, Russula, Septobasidium, and Tremella s.lat. (Zamora et al. 2014, 2016, Vidal et al. 2019). This result, together with the phylogenetic position of Dacryonaema, allowed inferring the bisterigmate basidium as the most probable ancestral state for the Dacrymycetes in most analyses. However, the rayDisc analysis assuming equal distribution of states at the root node unexpectedly reconstructed the 1-spored basidium as the ancestral state for the Dacrymycetes. This is the least parsimonious option and also the equally-distributed states assumption is anyway unrealistic.

McNabb (1973) emphasised the degree of branching of the hyphidia for delimiting species within Dacrymyces s.lat., and this is still used to characterise some species, e.g., Dacrymyces dendrocalami (Oberwinkler \& Tschen 1989, Shirouzu et al. 2009). In our analyses, the morphology of the hyphidia has been simplified to only two extremes (absent or simple vs conspicuous and branched) to be able to make use of imprecise descriptions from the literature. For instance, McNabb (1973) commented on the presence (sometimes occasional) of simple hyphidia in the majority of Dacrymycetes, in which branched hyphidia are not found, while Oberwinkler (2014) reported that the hymenium is composed of only basidia, a few species excepted. This character should be revisited in the future, since there are some clades, e.g., the one including $D$. chrysocomus and $D$. ovisporus, where hyphidia are often distinct, yet simple. In others, e.g., in Calocera s.str., and the D. chrysospermus and $D$. stillatus clades, true hyphidia are probably absent and the observed hyphae tend to develop into new basidia and new generative hyphae. While the plesiomorphic state in Dacryonaema and Unilacryma seems to be the branched hyphidia, and the ancestral state for Dacrymycetaceae the simple or absent hyphidia, important differences are found between the ancestors of the Dacrymycetes and Cerinomycetaceae. These differences are related to both the type of phylogenetic trees and the method 
(Table 5-6), a relatively unusual result (Litsios \& Salamin 2012, Cusimano \& Renner 2014) that should be considered before drawing definitive conclusions. Consequently, given the current data, we consider the ancestral state of the hyphidia in the Dacrymycetes and Cerinomycetaceae to be uncertain.

There are additional characters that should be investigated in the future. Reid (1974) found that the basidium in Calocera furcata usually has an apical protuberance that is normally absent in $C$. cornea. This character was also noted by Van de Put (2014). The morphology of mitotic spores, whether dikaryotic arthrospores, other conidia formed from vegetative cells, or microconidia formed from the basidiospores, seems to be useful for species delimitation. Shirouzu et al. (2009) reported clear differences in mitospore morphology in fruitbodies of $D$. pinacearum, D. stillatus, and D. subarcticus, and Kirschner \& Yang (2006) described morphologically characteristic stauroconidia in Dacryoscyphus chrysochilus. Reid (1974) described Calocera pallidospathulata by emphasizing the deviant cylindrical microconidia produced by mature basidiospores when compared with other species of Calocera, which normally produce rounded microconidia. These traits and others (e.g., characteristics in culture) are promising for delineating taxonomic groups, especially within the Dacrymycetaceae.

\section{Phylogenetic relationships and boundaries among Dacrymycetes higher-level taxa}

\section{Differences among phylogenetic analyses}

Coalescence-based phylogenetic inference often outperforms concatenation when discordance among single-locus phylogenies exists, assuming that the conflicts are a consequence of incomplete lineage sorting (Pamilo \& Nei 1988, Kubatko \& Degnan 2007, Degnan \& Rosenberg 2009, Fernández-Mazuecos et al. 2018). Precise estimates of the effective population sizes, through inclusion of several individuals per species, benefit the species tree inference (Heled \& Drummond 2010), while the superior accuracy of coalescence vs concatenation is often only relevant for relatively short branches (Ogilvie et al. 2016). With our final dataset containing no incongruence after the exclusion of Dacryonaema RPB1 sequences, less than ten independent loci, and some species being represented by a single individuum, both analytical approaches supported approximately the same groups. The few alternative relationships among some species were unsupported and affected very short branches, particularly in the Dacrymycetaceae. On the other hand, despite the improvements in performance in StarBEAST 2, species tree inferences often require very long runs for the MCMC to converge (Ogilvie et al. 2017). Our RWTY analyses indicate that species tree topologies did indeed reach convergence, and we only noted some convergence problems with the speciation rate parameter (cySpeciationRate, with $100<$ ESS < 200), an observation made previously (Dorsey et al. 2018).

Phylogram and chronogram topologies obtained in the present study are generally congruent with the most recent phylogenies based only on data from the nrDNA (e.g., Shirouzu et al. 2016, 2017), at least concerning the supported groups, and slightly deviate from previous studies including fewer taxa (Shirouzu et al. 2013). Our improved resolution was probably achieved by the combination of more taxa and DNA loci.

\section{Taxonomic boundaries at ordinal and familiar ranks}

Nowadays, there are two main currents for describing higher level taxa. One is the most classical, focused on the diagnosability of the resulting named groups (Christenhusz et al. 2015) considering their evolutionary relationships (thus, in agreement with phylogenies based on molecular data), as followed by, e.g., the consortium of experts in The Angiosperm Phylogeny Group
(2016). The alternative method is 'temporal banding', which considers estimated stem ages of the clades to define taxonomic ranks (Avise \& Johns 1999, Holt \& Jønsson 2014). However, adhering too tightly to the temporal banding method may have undesirable taxonomic and nomenclatural consequences. For instance, it may force the joining, under the same higher-ranked taxon name, of lower-ranked taxa that are strikingly different based on phenotypic characters, thus resulting in heterogeneous assemblages that are difficult to recognise. Conversely, it could force the splitting of morphologically homogenous groups for little gain (Lücking 2019).We think that trying to reconcile the systems may be the best solution, i.e., proposing only diagnosable groups while considering their stem ages with some flexibility. As guides for our study, the mean stem age estimates proposed by Kraichak et al. (2018) is in the range 176-194 Ma for orders and 111-135 Ma for families in the Lecanoromycetidae and Ostropomycetidae (Ascomycota). Zhao et al. (2017), using a wide sampling in the Basidiomycota, reported stem ages of most orders around 120-290 Ma. Although Zhao et al. (2017) were not focused on the family level, the oldest crown ages estimated for families were c. 150-160 Ma (e.g., in Moniliellaceae and Phleogenaceae), meaning that their stem ages must be even older and broadly overlapping with the order ages. Considering these premises, we can circumscribe at least five groups within the Dacrymycetes that deserve a taxonomic rank above the generic level, agreeing with the current use of ranks in the Basidiomycota. Four of them are well characterised by phenotypic characters and highly supported monophyletic groups in all analyses, three of which are already named as families, viz., Cerinomycetaceae, Dacrymycetaceae, and Unilacrymaceae. The specimen NBRC 110597 may represent another family judging from its phylogenetic position, but since we lack morphological data and its phylogenetic placement is based only on nrDNA data from a single sample, we consider it appropriate to leave it unnamed for the time being

The clade containing the genus Dacryonaema includes taxa with long medallion clamps concentrated in the subhymenium and the bases of basidia and hyphidia, with septa normally lacking clamp connections elsewhere. The cortical/marginal hyphae are variable but some terminal cells are always abundantly branched and anastomosed, becoming gelatinised in $\mathrm{KOH}$. Hyphidia are branched and carotenoids are inconspicuous or absent. Basidiospores are \pm cylindric-allantoid and may remain aseptate or become transversely 1-septate (but rarely up to 3-septate) at maturity. In addition, all TEF-1 $\alpha$ sequences have an intron (the sixth in the analysed fragment) that is absent in other samples among the Dacrymycetes. This clade is proposed here as a new family, Dacryonaemataceae, since its morphological characteristics do not allow the placement of Dacryonaema in any of the previously circumscribed families. This decision is also in agreement with the relatively old stem age of the clade, as our results indicate that the ancestors of Dacryonaemataceae and Unilacrymaceae may have split around $169 \mathrm{Ma}$. This estimate was calculated setting an age for the Dacrymycetes within the ranges recently obtained by He et al. (2019) and Varga et al. (2019).

The clade representing the family Unilacrymaceae, which only contains the genus Unilacryma, is comprised of species with short clamp connections at most septa and often simple and not swollen terminal cells of marginal hyphae that are not highly gelatinised in $\mathrm{KOH}$. Hyphidia are branched and there is a high lipid content in the cytoplasm of the basidia and basidiospores. Carotenoids are inconspicuous or absent. The basidiospores are ovoid to ellipsoid, presumably uninucleate when young, and become muriform at maturity. The discovery of the new species $U$. bispora demonstrates that simple basidia, one of the main morphological characters used to recognise the family by 
Shirouzu et al. (2013), is not a synapomorphy of this group. On the other hand, the presence of ovoid-ellipsoid basidiospores that become muriform, another important character noted in Shirouzu et al. (2013), is also not enough to distinguish the family, since this morphology is present in Dacrymyces ovisporus (Fig. 5s). This species, however, is a typical member of the Dacrymycetaceae and is characterised by the conspicuously high carotenoid cell content, and is additionally distinguished from Unilacryma by the simple to (rarely) sparingly branched hyphidia (Fig. 2I). In addition, D. chrysocomus, a close relative to $D$. ovisporus in our phylogenetic analyses, has predominantly cylindric-allantoid basidiospores that frequently become muriform at maturity. Scattered shorter, ellipsoid basidiospores may be present, linking the morphology of the cylindric-allantoid basidiospores with the ovoid ones.

The clade representing the family Cerinomycetaceae contains species with short clamp connections at most or all septa and often simple and not swollen terminal cells of cortical/marginal hyphae that are not clearly gelatinised in $\mathrm{KOH}$. The hyphidia are often abundantly to sparingly branched, but sometimes simple or absent. The amount of lipid bodies in the cytoplasm of basidia and basidiospores is normally low (with one exception), and carotenoids are inconspicuous or absent. The basidiospores are \pm cylindric-allantoid, binucleate during some time before the formation of the first septum, and may remain aseptate or become transversely 1-3-septate at maturity. Basidia tend to be relatively short compared to those of the other clades, especially to Dacryonaema and Unilacryma. The fruitbody macromorphology varies from resupinate, coalescing to dacrymycetoid, and the consistency is \pm dry, fleshy-cartilaginous or gelatinous.

The clade representing the family Dacrymycetaceae includes several genera, e.g., Calocera, Dacrymyces, Dacryopinax, Femsjonia, and Heterotextus. Some of these genera are non-monophyletic, which has also been demonstrated in previous studies (Shirouzu et al. 2007, 2009, 2013). This clade is very species-rich and morphologically diverse, containing species with both clampless and clamped septa, variously shaped terminal cells of cortical/marginal hyphae. Basidiospore shape and septation are highly variable, although basidiospores tend to be cylindric-allantoid in most cases. All species studied possess basidia and basidiospore cytoplasm with high lipid contents and young basidiospores with a single nucleus before the formation of the first septum. The presence of conspicuous carotenoids and the absence of highly branched hyphidia are characteristic of most species, but exceptions occur. All TEF-1a sequences obtained for this family lacked the third intron in the analysed fragment.

Taxa at the order level are not well characterised, and some doubts arise when assessing the placement of Dacryonaemataceae. A conservative classification that would agree with the tree topology and partly with the estimated node ages, and in addition does not require any nomenclatural changes, would be to adopt the two orders proposed by Shirouzu et al. (2013) and include Dacryonaemataceae in the order Unilacrymales. However, since the 1-spored basidium is no longer a synapomorphy of the Unilacrymales, and since basidiospore shape would also not be diagnostic with the putative inclusion of Dacryonaemataceae, the phenotypic characterization, i.e., diagnosability, of Unilacrymales becomes problematic. Some characters are shared by the Dacryonaemataceae and Unilacrymaceae, e.g., the clamped hyphae, branched hyphidia, absence of conspicuous carotenoids, and the similar ecology, but those traits are to some extent shared with members of the Cerinomycetaceae. The amount of observable lipids is not diagnostic, as no lipid drops are seen in the cytoplasm of $D a$. rufum. As noted above, basidia are often much larger in Dacryonaemataceae and Unilacrymaceae when compared with
Cerinomycetaceae, but in the Dacrymycetaceae some species also have relatively large basidia. The differences between $\mathrm{Ce}$ rinomycetaceae and Dacrymycetaceae are also considerable, making it a challenge to identify diagnostic characters that can include all members of both families while at the same time excluding all taxa in Dacryonaemataceae and Unilacrymaceae. Consequently, sorting the four named families into two orders seems unpractical.

There are two alternatives instead of accepting the two currently recognised orders in the Dacrymycetes. One would be to create a new order for Dacryonaemataceae, and the second option to accept the four families (putatively five) within a single order Dacrymycetales. Erecting an order for Dacryonaemataceae might be justifiable from both a morphological point of view and by using the divergence times from Zhao et al. (2017). However, it would then be logical to also include the Cerinomycetaceae in a separate order, resulting in the recognition of four orders, with a single family in each one. We refrain from taking this action in the present study, as it would currently not provide any substantial benefit. The recognition of the four described families in a single order Dacrymycetales is the other conservative approach that does not require any nomenclatural change, and seems reasonable based on morphological and molecular data. Considering the ample overlap among stem and crown ages of orders and families in Zhao et al. (2017), and the uncertainty of our age estimates, we believe that this provisional decision does not disagree with the temporal banding method. Further studies based on genome-scale data could provide a more definite classification for the higher ranked taxa, as well as more accurate age estimates.

\section{Taxonomy below the family level and future directions}

Relationships among species groups in the Dacrymycetaceae are not well resolved, and generic delimitations and species circumscriptions need substantial efforts to reach taxonomic and nomenclatural stability. Subclade D1 includes $D$. chrysocomus s.lat., $D$. ovisporus, and $D$. variisporus, and is characterised by the presence of clamp connections, thick-walled terminal cells of cortical/marginal hyphae, which may form a cortex concolorous with the hymenium, and by the large basidiospores that are either transversely multiseptate and/or muriform at maturity. Subclade D2 corresponds to Heterotextus ( $H$. miltinus s.lat., generic type) and has clamp connections, a cortex of thickwalled dilated terminal cells, concolorous with the hymenium, and multiseptate but not muriform, basidiospores. Subclade D3 is formed by a group of sessile to stalked Dacrymyces s.lat. with clamp connections and 0-3-septate, thin-walled basidiospores $(D$. ancyleus, $D$. citrinus, $D$. cylindricus, and D. cyrtosporus). Subclade D4 contains a group of stalked species of Calocera s.lat., Dacrymyces s.lat. and Dacryopinax s.lat. with clamp connections and thin-walled, 0-3-septate basidiospores (Calocera guepinioides s.lat., Ca. fusca, D. flabelliformis, and Dacryopinax sphenocarpa). Subclade D5 is a morphologically heterogeneous group with sessile and stalked species, with and without clamp connections, with or without branched hyphidia and with thin- to slightly thick-walled, 0-3-septate basidiospores, including $\mathrm{Ca}$. arborea, D. dendrocalami, and D. cf. adpressus. Subclade D6 corresponds to the genus Femsjonia (F. monospora and F. peziziformis s.lat., generic type), which has clamp connections, a contrasting pale cortex of thin- to thick-walled, cylindrical or \pm dilated terminal cells, and 1-multiseptate, thin-walled basidiospores. Subclade D7 includes specimens provisionally identified as $D$. minutus, with clamp connections, a cortex of thick-walled and \pm dilated terminal cells, concolorous with the hymenium, and 3-septate basidiospores. Finally, subclade D8, the 'core' Dacrymycetaceae, includes most species without clamp connections (with the exception of $D$. pachysporus), and the generic types of 
Calocera (Ca. viscosa), Dacrymyces (D. stillatus), Dacryomitra (Dacryomitra pusilla), Dacryopinax (Dacryopinax elegans), Dacryoscyphus (Dacryoscyphus chrysochilus), Ditiola (Di. radicata), and Guepiniopsis (G. buccina). The macromorphology in this large last group is highly variable, with fruitbodies that may be resupinate, clavarioid, cochleariform, flabelliform, pulvinate, etc., and with thin- to thick-walled basidiospores displaying various septation patterns. While some groups in subclade D8 are morphologically homogeneous, e.g., Cerinomyces without clamp connections, or the $D$. chrysospermus group with multiseptate basidiospores, there are also exceptions. For example, $D$. novae-zelandiae s.lat. and $D$. san-augustinii, which also have multiseptate basidiospores, are phylogenetically related to Dacryopinax elegans, a species with 3-septate, thick-walled basidiospores and a very different macromorphology. The lack of support for some of these groups is likely worsened by the limited amount of available DNA data. With the primers and PCR programmes provided in the present study, it should be unproblematic to obtain at least three or four unlinked DNA loci from recently collected ( $\leq 20$ years old) samples.

The inclusion of several new samples in our Dacrymycetes phylogeny has led us to detect some misidentifications that have taxonomic and nomenclatural relevance. For example, D. stillatus and Di. radicata, the types of two accepted genera, have been variously interpreted in the literature. Dacrymyces stillatus is likely a species complex, and an improved sampling of taxa and markers is needed to clarify its identity. The specimens provisionally identified here as $D$. stillatus s.str. agree well with the protologue and with the current European concept of the species, which is a taxon very commonly found in the Nordic countries. This species has clear molecular differences with the 'D. stillatus' sample used by Shirouzu et al. (2013), and also a somewhat different morphology according to the published description and iconography. Ditiola radicata is a common species in Europe, and the Swedish and Finnish specimens included in our phylogeny agree very well with the general concept of this species. However, this understanding of the species is notably different from 'Di. radicata' sensu Shirouzu et al. (2013), obtained from culture HHB-12010, which nests in the same clade as specimens previously identified as $D$. capitatus and $D$. lacrymalis. This clade is probably another species complex, within which $D$. capitatus s.lat. displays substantial morphological variation and misidentifications are likely. The sequences retrieved from the JGI as 'Calocera cornea' were also substantially different from those of our samples of $\mathrm{Ca}$. cornea and agree well with preliminary data we have obtained from our own specimens of Ca. furcata. The present study is not aimed at resolving the taxonomy of these taxa, but the disagreements mentioned here serve to caution against uncritical taxonomic rearrangements and nomenclatural novelties based on previously generated data.

\section{Species delimitations in Dacryonaema and Unilacryma}

According to our results, the five species of Dacryonaema and Unilacryma found in the Nordic countries seem to be well differentiated, and distinguishing them by morphological or molecular data (or both) is unproblematic. The three species of Dacryonaema accepted here are highly supported by the STACEY species delimitation analysis, receive high support in all other phylogenetic analyses (Fig. 10), and are distinguished by several morphological characters (see Taxonomic part). Therefore, we do not consider them mere infraspecific divergent populations (Sukumaran \& Knowles 2017). In addition, distribution ranges overlap at least in northern Europe, yet the three species retain their morphological distinguishing features in sympatry. little taxonomic problems except for its potential confusion with
Dacrymyces ovisporus (see Taxonomic part). The STACEY analysis supported this as a distinct species, and it appears monophyletic in all phylogenetic analyses. Furthermore, basidia and basidiospore morphology (Fig. 6, 14-15) clearly separate it from its closest relative, $U$. unispora. Species delimitations in the Unilacryma unispora s.lat. clade are much less clear. While, as a group, it is distinguished from $U$. bispora, it is not entirely clear whether more than one species should be recognised here. As noted in the results, the $U$. unispora s.lat. clade is formed by two partially supported subclades, one joining all North European specimen and the other comprising the two Japanese specimens and additional environmental sequences from eastern Asia. It should be noted that the only molecular difference between the two clades shared by all included samples are two transitions $(\mathrm{C} / \mathrm{T})$ in the $28 \mathrm{~S}$ dataset. Additional molecular differences are present between the 18S, ITS, and $R P B 2$ sequences of the Japanese specimens and those from the Nordic countries. Even if the two clades are distinguished by STACEY as two putative species, this pattern can easily be obtained when analysing few samples of isolated populations in species with a broad distribution (Sukumaran \& Knowles 2017, Chambers \& Hillis 2019). Also, the few differences detected might just be part of their intraspecific variation. Furthermore, the clade formed by the Japanese samples is poorly supported in the concatenated Bayesian analysis, although it is well-supported in the ML analysis. Since we have no information about other populations of $U$. unispora s.lat. in Eurasia and North America, and the amount of missing data from the East Asian clade is high due to the single region obtained from environmental studies, keeping all of them temporarily as a single species is the only justifiable option with the present data. A phylogeographic study and a careful morphological analysis involving numerous vouchered samples from throughout the northern hemisphere is needed to decide whether $U$. unispora is a single species or a complex of closely related species.

\section{TAXONOMY}

We present the following provisional key to families in the class Dacrymycetes:

1. Cortical/marginal hyphae with terminal cells branched and frequently anastomosing, not conspicuously dilated, at least some of which become heavily gelatinised in $\mathrm{KOH}$; most hyphae thick-walled and clampless; subhymenial hyphae \pm thin-walled, with long, often angulose or slightly spurred, medallion clamp connections, present also at the bases of basidia and hyphidia, but very sparse or absent elsewhere

. Dacryonaemataceae

(currently a single genus, Dacryonaema)

1. Cortical/marginal hyphae with terminal cells not or only sparingly branched, without or with disperse anastomoses, rarely becoming gelatinised in $\mathrm{KOH}$ (and then well-defined and conspicuously dilated); hyphae thin- or thick-walled, either all clampless or with numerous clamp connections in most parts of the fruitbodies (sometimes inconspicuous at internal hyphae); clamp connections, when present, with variable morphology, but not consistently long and angulose if of medallion type

2. Basidiospores \pm ovoid-ellipsoid, soon with a single complete transverse septum (very rarely $2-3$ ), becoming muriform due to the formation of additional oblique, longitudinal and partial transverse septa; without conspicuous carotenoid contents (spore print whitish to pale yellowish cream) observable under the light microscope in any cell; hyphidia distinct and abundantly branched

Unilacrymaceae 
2. Basidiospores commonly cylindric-allantoid and aseptate or with only transverse septa, very rarely ovoid-ellipsoid and becoming muriform, but then with visible carotenoid contents when fresh (spore print often yellow or orange) that are also present in other cells (basidia, etc.), and with indistinct or simple hyphidia $\ldots \ldots \ldots \ldots \ldots \ldots \ldots \ldots \ldots$

3. Basidiospores with $0-3$ transverse septa, never with longitudinal or oblique septa, thin-walled, all or several binucleate when still aseptate; basidia and basidiospores normally without a conspicuously granular/vacuolar content when observed in $\mathrm{KOH}$, but sometimes with some granules that may gradually coalesce in dead state to form small (normally $<2 \mu \mathrm{m}$ diam) lipid drops, exceptionally with a higher lipid content; cells without conspicuous carotenoid contents; clamp connections always present; basidiomata resupinate to pulvinate, never long stalked ..... . Cerinomycetaceae

(currently a single genus, Cerinomyces)

3. Basidiospores with 0-many transverse septa and sometimes with additional longitudal or oblique septa, thin- to thick-walled, uninucleate when young and still aseptate; basidia and basidiospores with a densely and conspicuously granular/vacuolar content when observed in $\mathrm{KOH}$, which gradually coalesces in dead state to form conspicuous (at least some > $2 \mu \mathrm{m}$ diam and often much bigger) lipid drops; cells frequently with distinct carotenoid contents, rarely secondary lost; clamp connections present or absent; basidiomata variously shaped.

Dacrymycetaceae (several genera)

Class Dacrymycetes Doweld, Prosyllabus Tracheophytorum, tentamen systematis plantarum vascularium (Tracheophyta): LXXVII. 2001

Family Dacryonaemataceae J.C. Zamora \& S. Ekman, fam. nov. - MycoBank MB833097

Type. Dacryonaema rufum (Fr. : Fr.) Nannf., Svensk Bot. Tidskr. 41: 336. 1947

Fruitbodies \pm firmly gelatinous when fresh, sometimes cartilaginous, xerotolerant, sessile or stalked, with the fertile parts pulvinate to depressed or subglobose. Hymenium amphigenous, or confined to the upper part of the fruitbody when fruitbodies are discoid, then with a distinct sterile cortex. Cortical/marginal hyphae variable, frequently clampless, but sparse loop-like clamp connections may occur, some with terminal cells clearly branched and anastomosing, strongly gelatinised in $\mathrm{KOH}$; unbranched cylindrical, thick-walled and slightly dilated terminal cells may also occur, then normally tapering at the apex. Internal hyphae thick-walled, clampless or with very sparse clamps. Subhymenium composed by more or less thin-walled hyphae, with long and angulose, medallion clamps. Basidia 2-spored, bearing a loop-like basal clamp connection; apex frequently U-shaped, sometimes $\mathrm{Y}$-shaped, and more rarely with an apical protuberance. Hyphidia abundantly branched and often anastomosing, with a loop-like basal clamp connection. Recently discharged and still aseptate basidiospores uninucleate. Mature basidiospores 0-1-septate (exceptionally 3-septate), thin-walled, hyaline, cylindric-allantoid. Spore print whitish. Microconidia ellipsoid. Cell cytoplasm without conspicuous carotenoids under the light microscope. Brownish intracellular and diffuse parietal pigments often visible at least in some cortical/marginal hyphae. Basidia and basidiospores with or without conspicuous lipid drops when old.

Included genera - Dacryonaema Nannf., Svensk Bot. Tidskr. 41: 336. 1947 (type).

\section{Key to species}

1. Fruitbodies for a long time appearing as a conical, dark brown to dark reddish brown (rarely amber-coloured), sterile stalk, which finally develops an apical, often rounded or irregularly shaped, but not centrally depressed, pruinose fertile head, $\leq 0.7 \mathrm{~mm}$ diam when fresh and mature; dead basidia and basidiospores with a rather even cytoplasm, with invisible or hardly discernible lipid bodies; basidiospores (2.9-)3.2-4.3(-4.9) $\mu \mathrm{m}$ wide . . . . . . . . . Da. rufum

1. Fruitbodies very variable in colour, from yellowish or cream coloured to brownish or dark brown, pulvinate to pustulate at first, later slightly convex or becoming flattened or centrally depressed (pezizoid), sessile or sometimes shortly stalked, often $\geq 0.7 \mathrm{~mm}$ diam when fresh and mature; dead basidia and basidiospores with a slightly to conspicuously granular cytoplasm, often with some discernible lipid bodies; basidiospores (3.5-)4.3-6.6(-7.1) $\mu \mathrm{m}$ wide

2. Fruitbodies yellowish, cream coloured or brownish; cell cytoplasm with distinct or indistinct lipid content; basidiospores on average $<14 \times 5.3 \mu \mathrm{m} \ldots \ldots \ldots \ldots$ Da. macnabbii

2. Fruitbodies brown to dark brown or blackish; cell cytoplasm normally with a rather distinct lipid content; basidiospores on average $>14.5 \times 5.3 \mu \mathrm{m} \ldots \ldots$. . macrosporum

Dacryonaema macnabbii (D.A. Reid) J.C. Zamora \& S. Ekman, comb. nov. - MycoBank MB833098; Fig. 11

三 Dacrymyces macnabbii D.A. Reid, Trans. Brit. Mycol. Soc. 62: 456. 1974 [basion.]

Typus. United KIngdom, Wester Ross, Kinlochewe, Coille na Glas-Leitire, on Pinus sylvestris, 20 Aug. 1963, R.W.G. Dennis (K(M) 81678! holotype).

Basidiocarps \pm firmly-gelatinous, from pale cream coloured to yellowish, ochraceous, or brownish, sometimes with an orange tinge, darker when dried, xerotolerant, at first erumpent or pulvinate, soon discoid, flattened to centrally depressed (pezizoid), sessile or shortly stalked, attached to the substrate by a central point and sometimes rooting, (0.5-)0.7-1.5(-1.8) $\mathrm{mm}$ diam when mature. Cortical/marginal hyphae conspicuous, variable, at least some with terminal cells abundantly branched and partially anastomosing, with walls that become gelatinised in $\mathrm{KOH}$; some slightly to clearly thick-walled and somewhat dilated cells may also appear. Internal hyphae (2-)2.5-7 $\mu \mathrm{m}$ wide, slightly to clearly thick-walled, often roughened, embedded in a gelatinous matrix, clampless or, rarely, with scattered and obscure long clamps. Hymenium usually confined to the upper surface of the fruitbodies, rarely becoming amphigenous with age, forming numerous basidia intermixed with hyphidia. Subhymenium with thin- to slightly thick-walled hyphae, with long and \pm angulose medallions, including at the bases of basidia and hyphidia. Hyphidia welldeveloped, profusely branched, normally abundant, 2-4(-5) $\mu \mathrm{m}$ thick in the upper half, terminal branches 1-2(-2.5) $\mu \mathrm{m}$ thick, often with simple septa throughout their length. Young basidia cylindrical to very narrowly clavate, sometimes with a tortuous base. Mature basidia 2-spored (very rarely 3-spored basidia have been seen), (42-)45-100(-125) × (4-)4.5-7(-10) $\mu \mathrm{m}$, sterigmata 11-20 $\mu \mathrm{m}$ long, often forming an obtuse angle or arising from slightly lateral position from the apex, basidia sometimes with a small apical papilla between the sterigmata. Basidiospores (8.7-)10.4-13.9(-15.4) × (3.5-)4.3-5.5(-6.2), $\mathrm{Q}=(1.6-) 2-3.2(-3.7)$, ellipsoid to cylindric-allantoid. Basidia and basidiospore cytoplasm hyaline, often slightly granular in $\mathrm{KOH}$ due to the presence of some lipid bodies, which may form small lipid drops after coalescence. Microconidia from germinating basidiospores rarely seen, hyaline, narrowly ellipsoid to cylindric, about 3.5-4.5 × 1-1.5 $\mu \mathrm{m}$. 

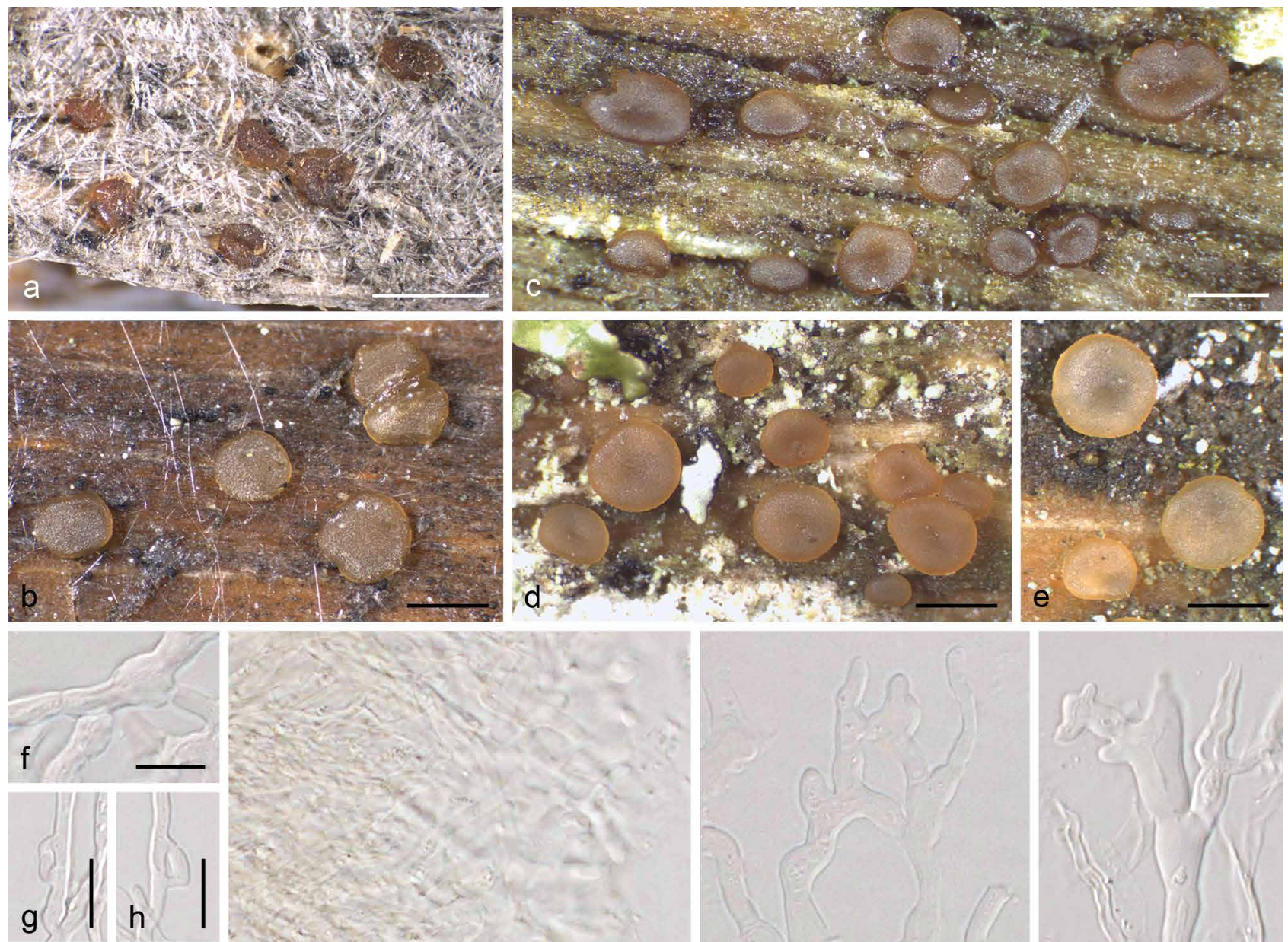

4
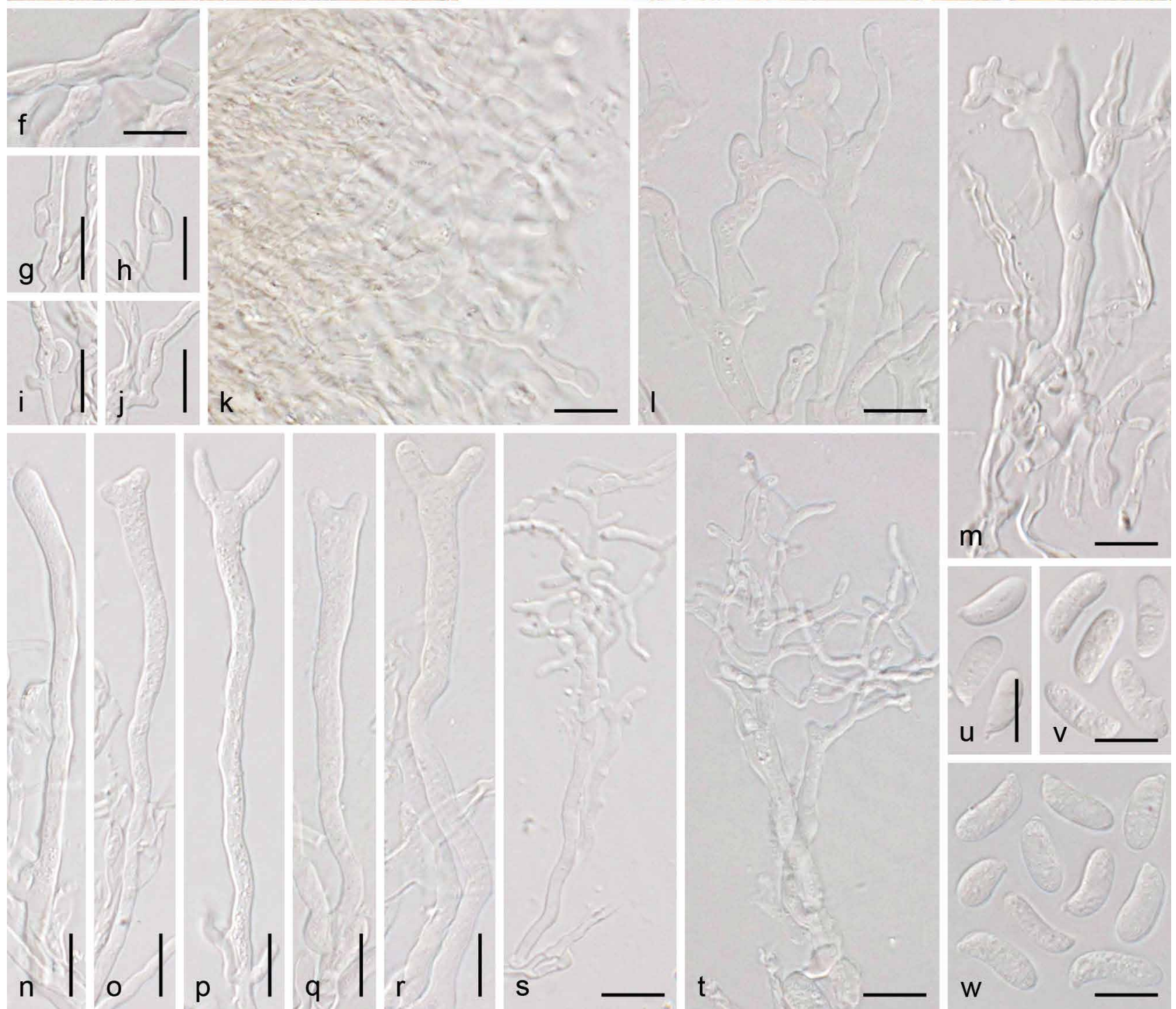

Fig. 11 Dacryonaema macnabbii. a-e. Fruitbodies in different degrees of development, all in fresh condition except a; f-w. Micromorphology; f. thick-walled, clampless internal hyphae; $\mathrm{g}-\mathrm{j}$. long medallion clamp connections; $\mathrm{k}$. gelatinised and branched terminal cells of cortical hyphae; I. anastomoses; $\mathrm{m}$. thick-walled and slightly dilated marginal hyphae; $n$. young basidium; $0-r$. basidia in different degrees of development; $s-t$. branched hyphidia; $u-w$. basidiospores. a, $u$. From the holotype; b, g-j, m-p, s, v. from UPS F-940950; c, k. from UPS F-940966; d-f, l, q-r, t, w. from UPS F-940954. - Scale bars $a-e=1 \mathrm{~mm}, \mathrm{f}-\mathrm{w}=10 \mu \mathrm{m}$. 
Ecology \& Distribution - Apparently common on exposed coniferous wood, particularly Pinus sylvestris branches still hanging from the tree, but also found on fallen wood lying on the ground. Its distribution is uncertain because it has been confused with Dacrymyces tortus and other species, but published records and studied specimens demonstrate that it is present in the temperate and boreal biomes of north-western Europe. We have studied specimens of Da. macnabbii from Finland, Norway, Sweden, and the United Kingdom. It has also been reported from Denmark by Mathiesen (1991), whose descriptions and iconography agree well with our observations. The only two Swedish specimens previously identified as $D a$. macnabbii (S 250593 and UPS F-561899) were misidentified and belong to the $D$. tortus group.

Nomenclature \& Taxonomy - The yellowish to brownish colour of the fruitbodies, as well as the abundantly branched hyphidia, the long loop-like clamp connections present at the bases of basidia, and the basidiospore dimensions, as already reported in the protologue (Reid 1974), easily distinguish this species. The holotype specimen is particularly well distinguished from the morphologically close $D a$. macrosporum thanks to its clearly ochraceous colour and the short basidiospores (10.5-13 $\mu \mathrm{m}$ as indicated by D.A. Reid). In addition, all sequenced samples accepted here as $D a$. macnabbii form a well-supported clade including Reid's type collection, clearly independent from the clade formed by all Da. macrosporum samples. Some species in the $D$. tortus group may be macromorphologically very similar to both $D a$. macnabbiiand $D a$. macrosporum, and a microscopical study is often needed to separate them. The cortical/marginal hyphae of $D$. tortus s.lat. are not finely and abundantly branched, their walls barely gelatinise in $\mathrm{KOH}$ (they may show a somewhat roughened surface), the hyphidia are less abundantly branched, basidia are smaller (particularly shorter), and clamp connections are much more abundant in all hyphae and not of the form of long or angulose medallions (short medallions may be present particularly on internal hyphae).

Additional specimens examined. FINLAND, Lapponia inarensis, Inari, near Inari Siida, N68.910836 ${ }^{\circ}$ E27.016711 ${ }^{\circ}$, on a woodplank, 15 Aug. 2018, J.C. Zamora \& P. Posio, UPS F-940997. - NorwAY, Akerhus, Urdal, NE-side of Buråstjern, PN 121,014, on Pinus sylvestris, 24 May 1981, Anna-Elise Torkelsen 33/81 (O 160027); Akerhus, Nes, Kleiva, Picea abies, 13 Sept. 1965, Anna-Elise Torkelsen 113/5 (O 160060). - SwEDEN, Blekinge, Augerum, Fjärdsjömåla, på granstock, 03 Dec. 1946, S. Wilkand 328 (UPS F-729787); Öland, Böda, Lindreservatet, N57.319448 ${ }^{\circ} \mathrm{E} 17.030849^{\circ}$, on Pinus sylvestris wood, 03 Oct. 2017, J.C. Zamora (UPS F-940992); Småland (Inre), Femsjö, Prästgårdsskogen, på murken barrträdsstam, 17 Oct. 1939, Seth Lundell 1739 (UPS F-730059); Östergötland, Gryt, c. 1000 m Ö om Forhem, ad ramos dejectos decorticatos pini, 23 Apr. 1946, J.Ax. Nannfeldt 8308 (UPS F-729963); Södermanland, Nacka, Nackareservatet, N59.28955 E18.17560 ${ }^{\circ}$ on Pinus sylvestris wood, 10 June 2018, J.C. Zamora, P. Posio \& J. Nieminen (UPS F-940966); Värmland, Lekvattnet, N60.163134 E12.71224 ${ }^{\circ}$, on Pinus sylvestris branch, 06 Oct. 2018, J.C. Zamora (UPS F-940986); Värmland, Lekvattnet, N60.172306 ${ }^{\circ}$ E12.699782 ${ }^{\circ}$, on Pinus sylvestris branch, 07 Oct. 2018, J.C. Zamora (UPS F-940990, UPS F-940991); Västmanland, Sala, Skuggan, Fiskarbo, grönmålat virke (stöd åt hängrönn), 07 July 1946, R. Morander 658 (UPS F-729778, collection mixed with $D$. tortus and other species); Uppland, Älvkarleby, Långsandsörarna island, N60.65783 ${ }^{\circ} \mathrm{E} 17.55504^{\circ}$, on Pinus sylvestris branches, $17 \mathrm{Apr}$. 2018, J.C. Zamora, S. Ekman \& M. Zuluaga (UPS F-940954, duplicate in H); Uppland, Uppsala-Näs, Lörsta skog, nära Skärfälten, på tallgren på marken, 24 May 1928, Seth Lundell 629 (UPS F-730091); Uppland, Uppsala, Norra Lunsen, N59.80680 E17.96804 ${ }^{\circ}$, on coniferous wood, probably Pinus sylvestris, 20 Aug. 2017, J.C. Zamora (UPS F-940951, UPS F-940952); Uppland, Uppsala, Norby skog, and morschen Pinus-Ästen am Boden, 10 Aug. 1928, S. Lundell 631 (UPS F-729789); Uppland, Uppsala, Norra Lunsen, N59.79390 E17.68667 ${ }^{\circ}$, on Pinus sylvestris wood, 19 Nov. 2017, J.C. Zamora (UPS F-940950); Uppland, Uppsala, Norra Lunsen, N59.790953 ${ }^{\circ} \mathrm{E} 16.687811^{\circ}$, on Pinus sylvestris branch, 30 Sept. 2018, J.C. Zamora, S. Ekman, A. Kruys \& D. Pfister (UPS F-940953); Uppland, Uppsala, Nåsten, ovanför Läbyvads anhalt, på murket tallgärdsel, 19 June 1931, S. Lundell 792 (UPS F-729790); Uppland, Uppsala, Stadsskogen naturreservat, Vallstigen, $\mathrm{N} 59.839763^{\circ} \mathrm{E} 17.626805^{\circ}$, on highly decomposed, probably coniferous, wood, 09 Apr. 2017, J.C. Zamora (UPS F-940949); Uppland, Viksta, strax NV om 'Stentorget', ad ramos dejectos decorticatos pini, 25 May 1947, J.Ax. Nannfeldt 9141 (UPS F-729994, collection mixed with $D$. tortus); Hälsingland, Enånger, Borka, N61.54875 $\mathrm{E} 17.04727^{\circ}$, on old Pinus sylvestris branch, 27 July 2018, J.C. Zamora (UPS F-940977); Medelpad, Borgsjö, Ånge, N62.54089 E15.70138 ${ }^{\circ}$, on old exposed Pinus sylvestris branch, 27 July 2018, J.C. Zamora (UPS F-940980); Jämtland, Mörsil, Klukshåckren, N63.172614 ${ }^{\circ} \mathrm{E} 13.600665^{\circ}$, on Pinus sylvestris branches, 23 July 2018, J.C. Zamora (UPS F-940993, UPS F-940994, duplicate in H); Jämtland, Mörsil, c. 2 km W of Sällsjö, N63.236875 ${ }^{\circ}$ E13.634149 , on old and weathered Pinus sylvestris branches, 26 July 2018, J.C. Zamora (UPS F-940995, UPS F-940996)

\section{Dacryonaema macrosporum J.C. Zamora \& S. Ekman,}

\section{sp. nov. - MycoBank MB833099; Fig. 12}

Etymology. The specific epithet 'macrosporum' refers to the large basidiospores compared to those of the two other Dacryonaema species described so far

Typus. FInLAND, Ostrobottnia ultima, Rovaniemi, N66.500201 ${ }^{\circ} \mathrm{E} 25.763870^{\circ}$, on Pinus sylvestris wood, 14 Aug. 2018, J.C. Zamora \& P. Posio (UPS F-941000! holotype, isotypes in UPS F-941001! and H!)

Diagnosis. Morphologically similar to Dacryonaema macnabbii, but fruitbodies frequently darker (brown to dark reddish brown or blackish), cell cytoplasm with more distinct lipid bodies (higher lipid content), and basidiospores distinctly larger, (12.9-)14.7-18.6(-23) × (4.4-)5.2-6.6(-7.1) $\mu \mathrm{m}$ (on average $>14.5 \times 5.3 \mu \mathrm{m})$.

Basidiocarps \pm firmly gelatinous, yellowish brown or ochraceous brown when young but soon becoming brown to dark brown or blackish, darker when dried, xerotolerant, at first erumpent or pulvinate, later discoid, flattened to centrally depressed (pezizoid), sessile or shortly stalked, attached to the substrate by a central point and sometimes rooting, (0.4-)0.7-1.5(-1.7) $\mathrm{mm}$ diam. Cortical/marginal hyphae variable, some abundantly branched and anastomosing terminal cells, with walls that gelatinise in $\mathrm{KOH}$, others slightly to clearly thick-walled and that may be partially swollen. Internal hyphae (2-)2.5-6 $\mu \mathrm{m}$ wide, slightly to clearly thick-walled, often roughened, embedded in a gelatinous matrix, and clampless, sometimes with scattered and obscure long clamps. Hymenium restricted to the upper surface of the fruitbodies, progressively forming numerous basidia intermixed with hyphidia. Subhymenium formed by thin- to slightly thick-walled hyphae, with common long and \pm angulose, medallion clamp connections, including at the bases of basidia and hyphidia. Hyphidia abundantly branched, $2-4.5 \mu \mathrm{m}$ thick in the upper half, terminal branches 1-2.5(-3) $\mu \mathrm{m}$ thick, often with simple septa throughout their length. Young basidia cylindric to narrowly clavate, sometimes with a tortuous base. Mature basidia 2-spored (3-spored basidia may be rarely seen), 65-112 $\times 5.5-7.5(-9) \mu \mathrm{m}$. Sterigmata $16-30 \mu \mathrm{m}$ long, often forming an obtuse angle or arising from slightly lateral position from the apex, rarely with an apical papilla. Basidiospores (12.9-)14.718.6(-23) × (4.4-)5.2-6.6(-7.1) $\mu \mathrm{m}, \mathrm{Q}=(2.2-) 2.4-3.3(-4)$, cylindric-allantoid. Basidia and basidiospore cytoplasm hyaline or, sometimes, with a subtle cream hue, normally with a \pm granular content in $\mathrm{KOH}$ due to the presence of lipid bodies, which may coalesce to form some distinct lipid drops. Microconidia from germinating basidiospores rarely seen, hyaline, narrowly ellipsoid to cylindric, about 3-4.5 × 1-1.5 $\mu \mathrm{m}$.

Ecology \& Distribution - Insufficiently known; it seems to be much rarer than Da. macnabbii and Da. rufum. All studied specimens were growing on rather weathered and decomposed Pinus sylvestris wood, on branches lying on the ground, where it may grow intermixed with species in the $D$. tortus group (e.g., in Fig. 11a, the slightly paler fruitbody partially shown at the bottom of the picture is $D$. tortus s.lat. 3 ).

Nomenclature \& Taxonomy - This species is morphologically distinguished from $\mathrm{Da}$. macnabbii by its darker colour and, 

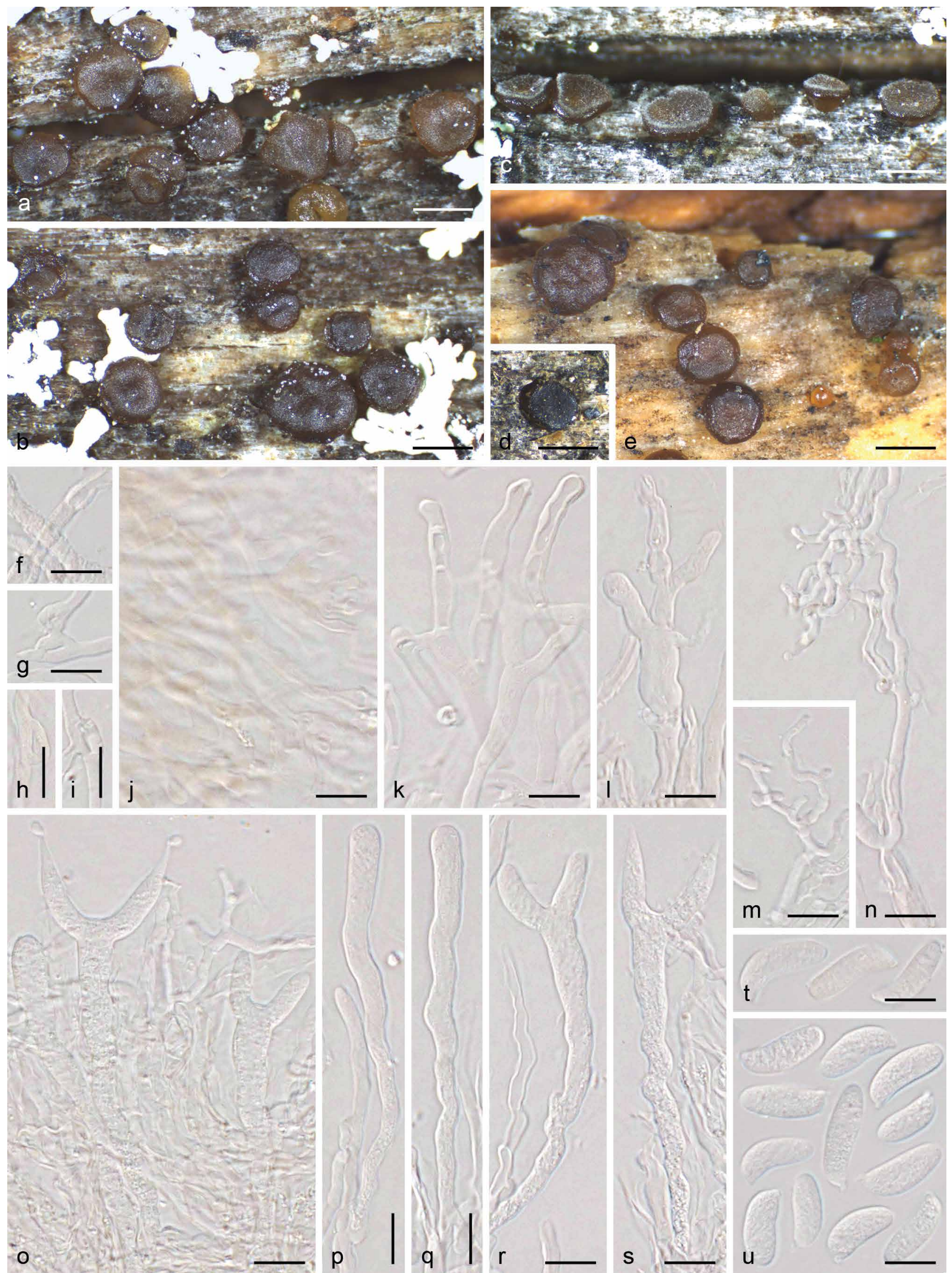

Fig. 12 Dacryonaema macrosporum. a-e. Fruitbodies in different degrees of development, all in fresh condition except d; f-u. Micromorphology; f. thickwalled, clampless internal hyphae; $g$-i. long medallion clamp connections; j. gelatinised and branched terminal cells of cortical hyphae; $k$. anastomoses; I. thickwalled and slightly dilated marginal hyphae; $\mathrm{m}-\mathrm{n}$. branched hyphidia; o. hymenium showing young basidia, mature basidia and hyphidia; $\mathrm{p}-\mathrm{q}$. young basidia; $\mathrm{r}-\mathrm{s}$. mature basidia in different degrees of development; $\mathrm{t}-\mathrm{u}$. basidiospores. a-c, f, h-j, I-s, u. from the holotype; g, k. from UPS F-941002.; d, t. from O 160045; e. from the isotype. - Scale bars $a-e=1 \mathrm{~mm}, \mathrm{f}-\mathrm{u}=10 \mu \mathrm{m}$. 
in particular, by the larger basidiospores. However, since the description above is based on only c. 250 fruitbodies (c. 150 from the holotype and isotype) from six individuals, its intraspecific variation is insufficiently known, so the traits selected as diagnostic characters should be taken with some caution. Lightcoloured specimens of $\mathrm{Da}$. macrosporum and dark coloured specimens of $D a$. macnabbii may be very difficult to separate, unless they are fertile and a certain number of well-developed basidiospores could be measured. Dacryonaema fruitbodies are comparatively long-lived and environmental conditions may affect basidiospore size, particularly if the maturation is aborted when the fruitbodies suddenly dry up. In addition, basidia in Da. macrosporum tend to be slightly wider and the amount of visible lipids in the basidia and basidiospore cytoplasm is slightly higher. Unfortunately, those characters show a considerable overlap and, rarely, specimens of $D a$. macnabbii with unusually wide basidia and a considerable amount of lipid bodies occur. In any case, the two species are clearly separated by DNA sequence data. For the distinction between $D a$. macrosporum and species in the $D$. tortus group, see taxonomic comments under Da. macnabbii. The two isotypes are part of the same individual, while the holotype is a different individual, collected on a different piece of wood. However, the three specimens are to be considered as duplicates because they are part of the same gathering (ICN, Art. 8.2, Turland et al. 2018).

Additional specimens examined. FINLAND, Ostrobottnia ultima, Rovaniemi, N66.499030 E25.763717 ${ }^{\circ}$, on Pinus sylvestris branches, 19 Aug. 2018, J.C. Zamora (UPS F-941002); Regio Kuusamoensis, Salla, Oulanka, Urriaavantie, N66.439727 ${ }^{\circ}$ E29.165568 ${ }^{\circ}$, on Pinus sylvestris branch, 17 Aug. 2018, J.C. Zamora (UPS F-940998, UPS F-940999). - NoRWAY, Hedmark, Kongsvinger, ved Sikåa mellom Møkeren og Varaldsjøen, on Pinus sylvestris, 27 May 1978, Anna-Elise Torkelsen 83/78 (O 160045).

\section{Dacryonaema rufum (Fr. : Fr.) Nannf., Svensk Bot. Tidskr. 41:} 336. 1947 - Fig. 13

三 Sphaeronaema rufum Fr., Kongl. Vetensk. Acad. Handl. 1818 [ser. 3, vol. 6]: 357.1818 [basion.] : Fr., Syst. Mycol. 2: 536. 1823.

इ Zythia rufa (Fr.) Schwein., Trans. Amer. Philos. Soc., n.s. 4: 247. 1834 [1832].

三 Sphaeronaemella rufa (Fr.) Sacc., Syll. Fung. 3: 618. 1884.

Typus. SwEDEN, [sine loc.], Scleromyceti Sueciae n. 27 (UPS F-117078! lectotype, designated here, MBT389286).

Basidiocarps firm-gelatinous to cartilaginous, xerotolerant, often consisting on a well-developed (but sometimes very short), reddish brown to dark reddish brown, narrowly conical stalk, $0.3-2(-2.5) \mathrm{mm}$ long, $0.3-0.7(-0.8) \mathrm{mm}$ wide at the base and tapering to around $0.2-0.4 \mathrm{~mm}$ wide near the apex. Tardily, an apical, concolorous or slightly paler, rounded, fertile head is developed, becoming pruinose and thus looking distinctly paler when fully mature, $0.3-0.8 \mathrm{~mm}$ diam. Fruitbodies sometimes orangish, amber-coloured or with a pinkish hue when growing in the dark or when developing and maturing quickly, distinctly shorter in the last case. External hyphae of the stalk typically thick-walled, strongly glued together and difficult to dissect; cortical/marginal hyphae variable, at least some with thin-walled, anastomosing and branched terminal cells, gelatinising in $\mathrm{KOH}$, others thin- to thick-walled and mostly unbranched, sometimes a few slightly dilated thickwalled terminal cells can be seen; cytoplasmic contents hyaline or with some brownish pigments; walls hyaline to slightly yellowish brown or brownish. Internal hyphae 3-10 $\mu \mathrm{m}$ wide, thick-walled, clampless or with very scattered and obscure long clamp connections, slightly embedded in a cartilaginous matrix and tightly adhering. Hymenium amphigenous, producing numerous basidia intermixed with somewhat scattered hyphidia. Subhymenium formed by thin- to slightly thickwalled (rarely clearly thick-walled) hyphae, bearing long and \pm angulose medallion clamp connections, including at the bases of basidia and hyphidia. Hyphidia distinct but sometimes sparse, branched, $2-4.5 \mu \mathrm{m}$ thick in the upper half, terminal branches 1-2.5 $\mu \mathrm{m}$ thick, often with simple septa throughout their length. Young basidia cylindric to narrowly clavate. Mature basidia 2-spored, 35-80(-85) × (2.5-)3.5-5.5(-6.5) $\mu \mathrm{m}$, sterigmata 9-23 $\mu \mathrm{m}$ long, often forming an obtuse angle or arising from slightly lateral position from the apex, rarely with an apical papilla. Basidiospores (8.7-)10.1-13.4(-14.8) $\times(2.9-)$ 3.2-4.3(-4.9), $\mathrm{Q}=(2.2-) 2.5-3.9(-4.9)$, cylindric-allantoid. Basidia and basidiospore cytoplasm hyaline, normally without visible lipid contents, rarely with scattered and minute lipid drops. Microconidia from germinating basidiospores hyaline, narrowly ellipsoid to cylindric, about 3.5-4.5(-5) × 1-1.5 $\mu \mathrm{m}$.

Ecology \& Distribution - Rather common and widespread, known from the temperate and boreal biomes of North America and Europe but not recorded from Eastern Asia (Shirouzu et al. 2009, 2016). It is apparently restricted to coniferous wood, mainly Pinus sylvestris in Europe, but also occurs on other genera and species (e.g., Pseudotsuga) in North America. It very frequently occurs on anthropic wood, and is rather widespread on fences, roofs, benches, etc. The ecology of this species was thoroughly detailed by Nannfeldt (1947).

Nomenclature \& Taxonomy - No specimens are cited, nor are illustrations or locality data present in the protologue of Sphaeronema rufum (Fries 1818). However, in the later Systema Mycologicum 2, Fries (1823) cited the exiccata 'Scleromyceti Sueciae n. 27'. According to the current Art. F.3.9 of the ICN, and since the name is sanctioned, those specimens are available for lectotype designation by being elements from the context of the sanctioning work. Two duplicates of Scleromyceti Sueciae n. 27 with the original label are kept in UPS. One is mounted on an envelope joined with other samples of the Scleromyceti Sueciae (first 100 numbers, UPS F-117472), and the other is mounted on a free envelope (UPS F-117078). The two specimens are conspecific, well-developed and typical, despite all fruitbodies being young and sterile. We chose UPS $\mathrm{F}-117078$ as the lectotype in order to facilitate future revisions. Since the selected lectotype is taxonomically unambiguous, the designation of an epitype is unjustifiable (ICN, Art. 9.9), regardless of, e.g., the lack of molecular data from this sample.

We have studied two further specimens collected by E. Fries. One (S F250639) was collected in Sweden without other locotypic indication, and is labelled as 'Original!' by a later hand than that of Fries. Both 'Sphaeronemella rufa (Fr.) Sacc.' and 'Sphaeria rufa Fr.' were written with the same ink and by the same hand, so the label was likely written after Saccardo (1884) made the combination in Sphaeronemella. The other specimen (UPS F-117079) was collected in Femsjö, but the label has no date and Femsjö is not cited as a collecting locality neither in the protologue nor in the sanctioning work. As a result, none of these specimens can be considered as original material.

The initially conical and typically dark reddish brown fruitbodies of Da. rufum have a unique morphology among all Dacrymycetes, being easily characterised in the field whether in its sterile or fertile state. Indeed, this species is not likely to be confused with any other Dacrymycetes, but some ascomycetes producing dark peritheca or pycnidia with long necks might appear similar to the naked eye. In fact, Fries $(1818,1823)$ placed various ascomycetes in the same genus and described the fruitbodies as perithecia. When fertile, Da. rufum is more difficult to confuse with any other species, although perhaps some confusion with calicioid fungi could arise. A careful examination under the microscope or even with a hand-lens would quickly dispel any doubt. Clamp connections in this species have been largely overlooked, even in the detailed study of Nannfeldt (1947), perhaps because they are normally restricted to the sub- 


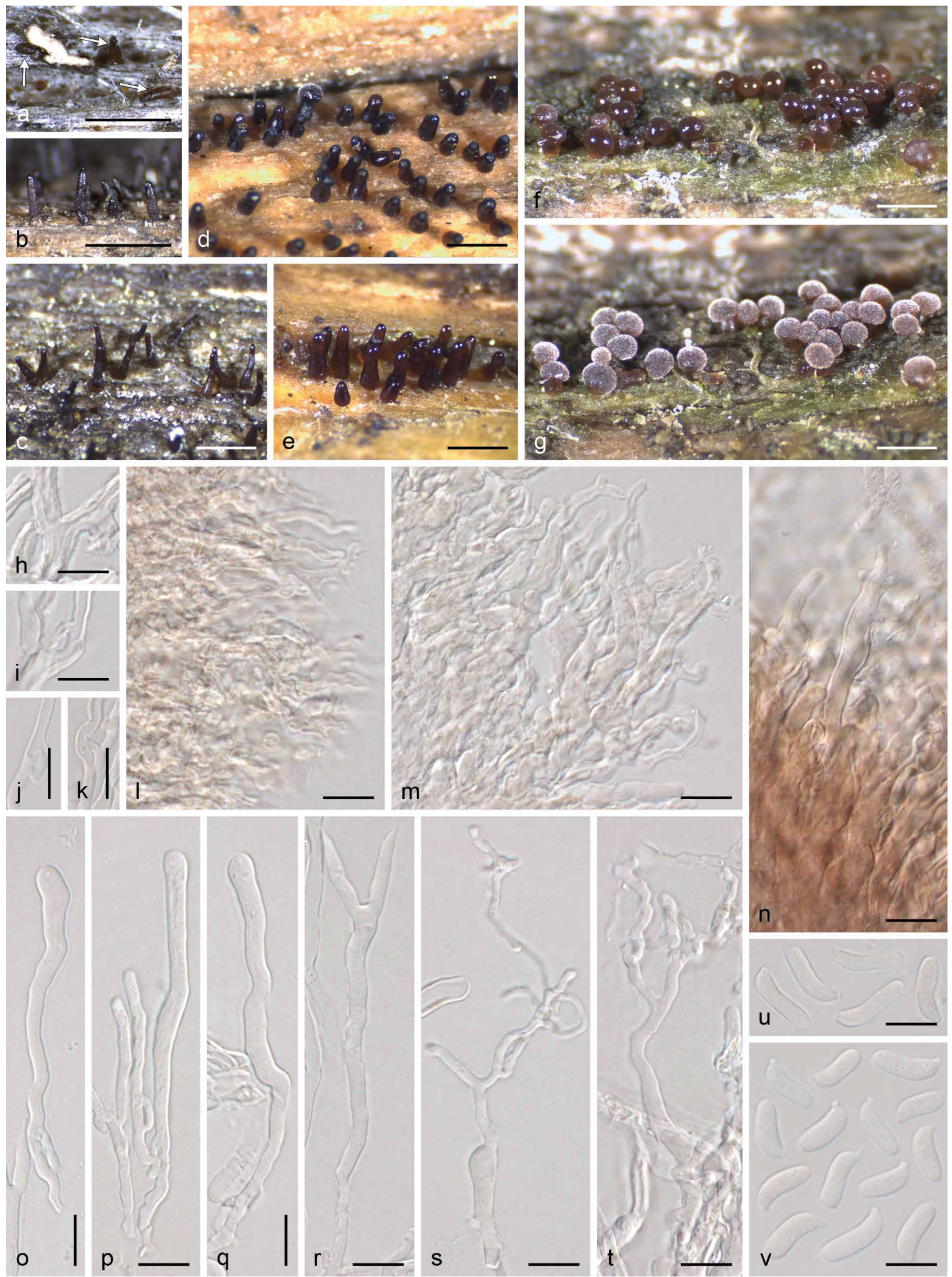

Fig. 13 Dacryonaema rufum. a-g. Fruitbodies in different degrees of development, all in fresh condition except a-c; $h-v$. Micromorphology; $h$. thick-walled, clampless internal hyphae; $\mathrm{i}-\mathrm{k}$; long medallion clamp connections; $\mathrm{I}-\mathrm{m}$. slightly gelatinised and branched terminal cells of marginal hyphae; $\mathrm{n}$. thick-walled and slightly dilated marginal hyphae; o-q. young basidia; r. mature basidium; s-t. branched hyphidia; $u-v$. basidiospores. a. From the lectotype; b. from TRTC 48002 (in UPS); c. from UPS F-941012; $d-s$, v. from UPS F-941005; $t-u$. from UPS F-941006. - Scale bars: $a-g=1 \mathrm{~mm}, \mathrm{~h}-\mathrm{v}=10 \mu \mathrm{m}$. 
hymenium and the bases of basidia and hyphidia, and because it is difficult to dissect $D a$. rufum specimens due to their hard texture. However, they were noted and accurately illustrated by Brough \& Bandoni (1975), but this was later considered as an exception in some North American specimens (e.g., Læssøe \& Petersen 2014). All fertile specimens of $D a$. rufum studied by us have conspicuous clamp connections at the bases of basidia, regardless of their geographical origin. The branched hyphidia were also not noted by Nannfeldt (1947), who stated: "no sterile elements have been seen in the hymenium". However, although they may be scarce and difficult to find in some samples, and several slides may have to be prepared to locate them, they are present in all fertile specimens studied by us. Brough \& Bandoni (1975) also found and illustrated branched hyphidia in the hymenium of this species.

Additional specimens examined (due to the high number of specimens studied, for European material we only cite those specimens used for morphological and molecular analyses, and typification, but c. 50 additional specimens, mainly from the Nordic Countries and preserved in O, S, and UPS, were revised as well). CANADA, Nova Scotia, Kings Co., Kentville, coniferous fence post, 17 Nov. 1954, K.A. Harrison (UPS s.n.); Ontario, Thunder Bay Distr., Black Sturgeon Lake, on old wood, 17 June 1977, A. Carter (TRTC 48002 in UPS). - FINLAND, Tavastia australis, Tammela, Mustiala, Salois (Saloinen), around P.A. Karsten's house, on a wood plank, 11 May 2018, J.C. Zamora \& P. Posio (UPS F-941012). - NorwAY, Hordaland, Voss, Mølstertunet, på gjerde, LN 583-585,246-248, 23 Sept. 1990, Anna-Elise Torkelsen 389/90 (O 295442); Troms, Storfjord, Skibotndalen, Lullesletta, DB 77,88, on Pinus sylvestris, 19 Aug. 1992, Anna-Elise Torkelsen 78/92 (O 295528). - SwEDEN, Skåne, Örkelljunga, Storeös mosse, on a wooden fence, 08 June 1977, Rolf Santesson 27333 (UPS F-730180); Uppland, Solna, Bergshamra, N59.38671 ${ }^{\circ} \mathrm{E} 18.02956^{\circ}$, on an old, fallen Pinus sylvestris tree, $01 \mathrm{Apr} .2018$, J.C. Zamora \& P. Posio (UPS F-941004, UPS F-941005); ibid., 08 July 2018, J.C. Zamora (UPS F-941006); Uppland, Uppsala, Norra Hagunda, Vänge, close to the parking at south of Fiby urskog naturreservat, N59.881589 E17.354279 , on a wood bench, 07 Apr. 2017, J.C. Zamora, M. Westberg, M. Svensson \& S. Ekman (UPS F-941003); Jämtland, Åre, Mörsil, Klukshåckren, N63.172614 ${ }^{\circ} \mathrm{E} 13.600665^{\circ}$, on Pinus sylvestris branches, 23 July 2017 , J.C. Zamora (UPS F-941011). - UnIted StATES of AmeRICA, Michigan, Mackinac Co., Big Knob Campground on Lake Michigan, N46.0333 ${ }^{\circ} \mathrm{W} 85.5833^{\circ}$, in a Thuja occidentalis swamp, 20 Aug. 1977, R. Santesson 27444 (S F250641); Oregon, Benton County, Mary's Peak, $914 \mathrm{~m}$, on decorticated branch of douglas fir, 26 Nov. 1983, B.R. Kropp (UBC F12372, UBC F12373).

\section{Family Unilacrymaceae Shirouzu, Tokum. \& Oberw., Mycologia} 105: 1120.2013

Type. Unilacryma unispora (L.S. Olive) Shirouzu, Tokum. \& Oberw., Mycologia 105: 1120. 2013.

Basidiocarps moderately to clearly gelatinous, but not of soft consistency, xerotolerant, pulvinate to depressed pulvinate when mature, with an almost amphigenous hymenium. Cortical/ marginal hyphae normally unbranched, \pm thick-walled, with terminal cells cylindrical to slightly tapering, not conspicuously swollen, with frequent clamp connections but also with some simple septa; anastomoses sparse. Context hyphae slightly to clearly thick-walled, with \pm rounded and short clamp connections visible at several septa, but some loop-like clamp connections and simple septa also often present. Subhymenium composed by thin to slightly thick-walled, clamped hyphae. Basidia 1- or 2-spored, bearing a basal clamp connection; when 2-spored apex often U-shaped, but basidia with an apical protuberance or with sterigmata forming an acute angle also occur. Hyphidia sparsely to abundantly branched, rarely anastomosing among them, with a basal clamp connection. Recently discharged and still aseptate basidiospores presumably uninucleate. Basidiospores subglobose to narrowly ovoid or ellipsoid, soon with one transverse septum and later becoming muriform due to the formation of additional longitudinal, oblique and sometimes transverse septa, thin-walled, hyaline or with a very subtle yellowish tinge. Spore print whitish. Microconidia ellipsoid. Cell cytoplasm without conspicuous carotenoids under the light microscope, at most, some basidia and basidiospores with a very subtle creamish tinge. Parietal diffuse yellowish brown pigments sometimes seen at some cortical/marginal hyphae. Basidia and basidiospores with conspicuous lipid bodies, becoming $\geq 2 \mu \mathrm{m}$ lipid drops at least in some old cells.

Included genera - Unilacryma Shirouzu, Tokum. \& Oberw., Mycologia 105: 1120. 2013 (type).

\section{Key to species}

1. Basidia 1-spored, 5-7.5 $\mu \mathrm{m}$ width; basidiospores (10.2-) 11.2-13.5(-15.7) $\mu \mathrm{m}$ width, $\mathrm{Q}=(1.1-) 1.2-1.4(-1.6)$, most of them ovoid to broadly ellipsoid ........ U. unispora 1. Basidia 2-spored, 6.5-8.5(-9) $\mu \mathrm{m}$ width; basidiospores (7.2-)8.2-10.4(-12.4) $\mu \mathrm{m}$ width, $\mathrm{Q}=(1.2-) 1.4-1.9(-2.2)$, most of them narrowly ovoid to ellipsoid ...... U. bispora

Unilacryma bispora J.C. Zamora \& Ekman, sp. nov. - MycoBank MB833100; Fig. 14

Etymology. The specific epithet 'bispora' refers to the 2-spored basidia, in contrast to the 1-spored basidia of the only other described species in the genus, $U$. unispora.

Typus. SwEDEN, Uppland, Uppsala, Norra Lunsen, N59.79390 E17.68667 ${ }^{\circ}$, on an old Pinus sylvestris branch, 19 Nov. 2017, J.C. Zamora (UPS F-941257! holotype, isotypes in UPS F-941258! and H!).

Diagnosis. Macromorphology similar to Unilacryma unispora, but basidia developing two sterigmata and thus producing two basidiospores, which are normally narrowly ovoid to ellipsoid, (7.2-)8.2-10.4(-12.4) $\mu \mathrm{m}$ wide, $\mathrm{Q}=(1.2-) 1.4-1.9(-2.2)$, instead of ovoid to broadly ellipsoid.

Basidiocarps \pm gelatinous, amber-coloured to brownish, xerotolerant, isolated, gregarious or crowded, initially erumpent, pulvinate, tardily becoming depressed-pulvinate, without a conspicuous margin, 0.3-1(-1.5) mm diam. Cortical/marginal hyphae slightly to clearly thick-walled, with cylindrical or attenuate terminal cells, (3-)3.5-7 $\mu \mathrm{m}$ wide, unbranched or rarely sparingly branched, with sparse anastomoses and both clamped and clampless septa. Internal hyphae 2.5-6 $\mu \mathrm{m}$ wide, slightly to clearly thick-walled, embedded in a gelatinous matrix, with clamp connections but also frequently with some simple septa. Hymenium almost amphigenous, progressively forming numerous basidia intermixed with some hyphidia. Subhymenium with thin to slightly thick-walled, clamped hyphae. Hyphidia hyaline, sparsely to heavily branched, $2.5-4(-4.5) \mu \mathrm{m}$ thick in the upper half, terminal branches (1-)1.5-2.5 $\mu$ m thick, often with clamped septa throughout their length. Young basidia narrowly claviform to subcylindric, tapering towards the base, $6.5-8.5(-9) \times 65-91 \mu \mathrm{m}$ when sterigmata start to grow. Mature basidia developing two sterigmata 19-33 $\mu \mathrm{m}$ long, often forming an obtuse angle, sometimes acute or with an apical papilla at the basidium apex. Basidiospores (10.4-)12.4-18.2(-19.7) $\times$ (7.2-)8.2-10.4(-12.4) $\mu \mathrm{m}, \mathrm{Q}=(1.2-) 1.4-1.9(-2.2)$, narrowly ovoid to ellipsoid, sometimes subfusiform, rarely ovoid or subcylindric, with a conspicuous 1.2-1.6 $\mu \mathrm{m}$ long hilar appendix, becoming transversely 1 -septate and tardily developing additional oblique, longitudinal and transverse septa, then becoming muriform, with a single hardly visible nucleus when recently discharged and still aseptate. Basidium and basidiospore cytoplasm hyaline or with a very pale yellowish tinge, with numerous small lipid bodies that slowly coalesce in dead state to form conspicuous lipid drops. Basidiospores germinating by the production of hyphae or microconidia. Microconidia narrowly ellipsoid to cylindric, about 3-4(-5) × 1.2-1.8 $\mu \mathrm{m}$, hyaline.

Ecology \& Distribution - Unilacryma bispora is a very common species that has only been observed fruiting on decorticated, old and frequently exposed wood of Pinus sylvestris, 

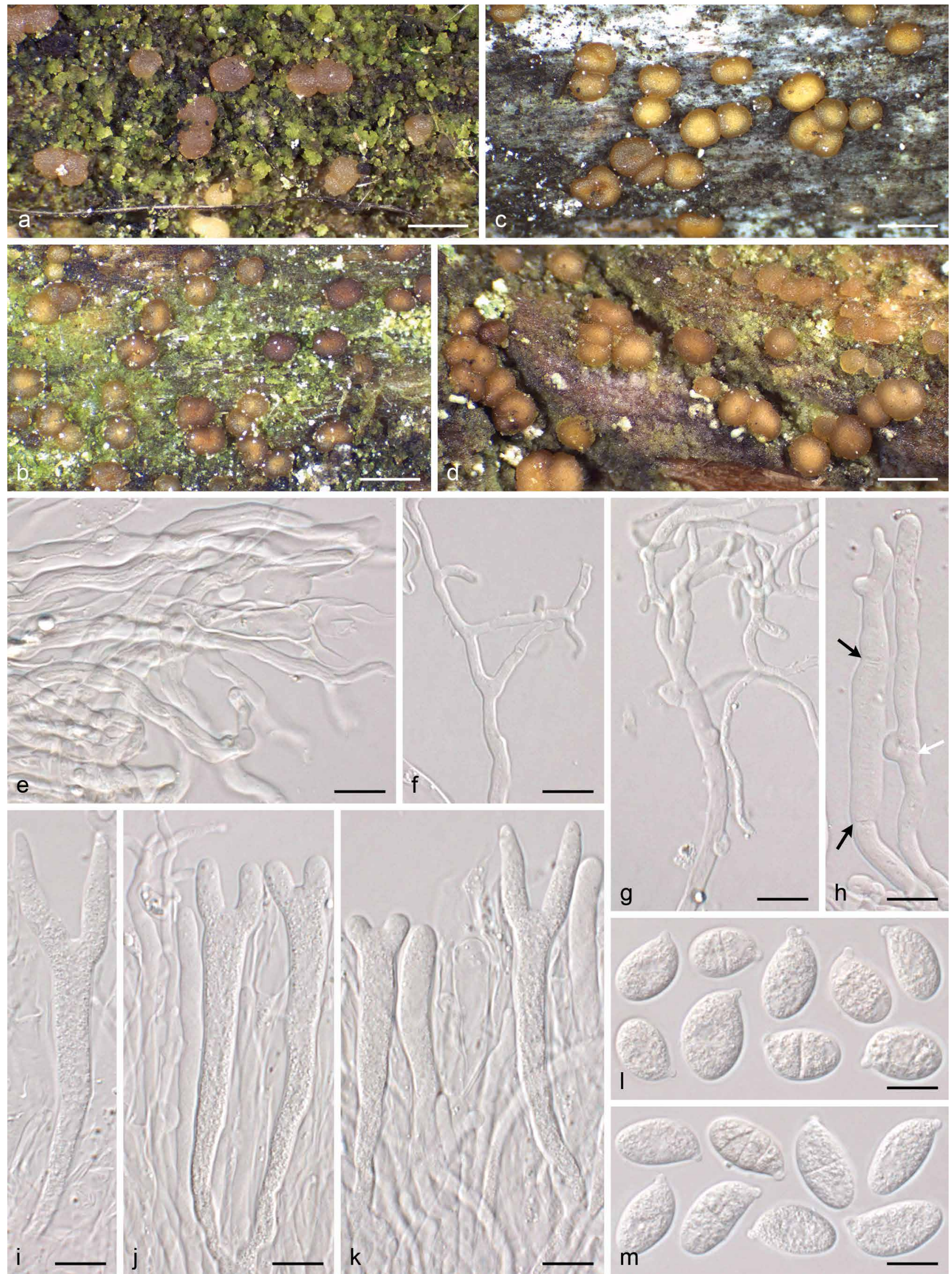

Fig. 14 Unilacryma bispora. a-d. Fruitbodies in different degrees of development, all in fresh condition; e-m. Micromorphology; e. poorly differentiated marginal hyphae; $\mathrm{f}-\mathrm{g}$. branched hyphidia; $\mathrm{h}$. terminal cells of marginal hyphae, note the presence of both clampless (black arrows) and clamped (white arrow) septa; i. basidium; j-k. hymenium showing young and mature basidia and hyphidia; I-m. basidiospores. a-b, e-g, i-I. From the holotype; c. from UPS F-941274; $\mathrm{d}$, h. from UPS F-941259; $\mathrm{m}$. from the isotype. - Scale bars: $a-d=1 \mathrm{~mm}, \mathrm{e}-\mathrm{m}=10 \mu \mathrm{m}$. 
especially on branches still attached to living trees. It occurs in both temperate and boreal forests, but it seems to be particularly common in the boreal biome, reaching the northern boreal zone. It is currently known from Finland, Norway, Russia, and Sweden. In a study on the fungal communities inhabiting Picea abies stumps (Kubart et al. 2016), environmental sequences probably related to this species were found in three Swedish nature reserves (Altarliden, Västerbotten; Fänstjärnskogen, Värmland; Ugglehöjden, Närke). The representative ITS sequence deposited in GenBank (KM494136) is identical to some of the ITS sequences of $U$. bispora generated in this study. Based on the results from the species delimitation analyses and considering the observed distribution in the field, we consider it very likely that those environmental sequences indicate that the species has a wider ecology than we have observed.

Nomenclature \& Taxonomy - Unilacryma bispora is readily distinguished by the combination of small, pulvinate and often amber-coloured basidiocarps, 2-spored basidia, ovoid to ellipsoid basidiospores that become muriform when mature, branched hyphidia, and absence of obvious carotenoids under the light microscope. It is similar to $U$. unispora except for the slightly broader, bisterigmate basidia, the slightly narrower and more elongate basidiospores, and the more northern distribution. The basidiospore shape is nevertheless somewhat plastic, and some specimens have predominantly ovoid basidiospores (e.g., UPS F-941254), while others show mostly ellipsoid ones (e.g., UPS F-941255). There may be little overlap among the length/width ratio of the basidiospores in certain specimens, but since most of them show intermediate shapes, and we have not detected any other differences, this should be regarded as part of the intraspecific variation. The holotype and the isotypes were collected on different, unconnected pine branches and are different individuals of the same gathering (ICN, Art. 8.2).

Following the literature and published identification keys (e.g., Torkelsen 1997), it might be confused with Dacrymyces ovisporus, a species with somewhat similar basidia and basidiospores, but with clearly larger, yellow-orange basidiocarps (Fig. $1 \mathrm{f}$ ), and with very obvious carotenoid contents in the cytoplasm of all cells, including the basidiospores (Fig. 5s). The comments by Oberwinkler (2014) on 'Dacrymyces ovisporus', indicating a macromorphology identical to that of Unilacryma unispora, and a very close micromorphology, might mean that the specimens studied by Oberwinkler actually belong to $U$. bispora. In its most commonly accepted sense, Dacrymyces ovisporus is additionally distinguished by often staining the substrate yellow (Torkelsen 1997), by hyphidia that are simple or rarely with 1-2 short branches (Fig. 2l), and by both the basidiospore shape and septation, which is more similar to that of $U$. unispora (thus, more rounded and becoming muriform earlier), this last character being well represented in the original plate of the protologue (Brefeld 1888). Further details on D. ovisporus will be provided in a forthcoming study. The two species are distantly related according to our phylogeny, belonging to two different families (Fig. 8-9).

Additional specimens examined. FINLAND, Nylandia, Espoo, near Nuuksio National Park, on a decorticated Pinus sylvestris branch, 12 May 2018, J.C. Zamora \& P. Posio (UPS F-941270); Tavastia australis, Tammela, near Saaren Kansanpuisto, on Pinus sylvestris branch, 11 May 2018, J.C. Zamora \& P. Posio (UPS F-941271); Ostrobottnia ultima, Rovaniemi, N66.49917 ${ }^{\circ}$ E25.767530 , on Pinus sylvestris branches, 14 Aug. 2018, J.C. Zamora (UPS F-941272); Regio Kuusamoensis, Salla, Oulanka, Urriaavantie, N66.439727 $7^{\circ} 29.165568^{\circ}$, on Pinus sylvestris branch, 17 Aug. 2018, J.C. Zamora (UPS F-941274, UPS F-941275); Lapponia sompiensis, Pelkosenniemi, Saunavaara, N67.049234 ${ }^{\circ}$ E27.524627 $7^{\circ}$, on Pinus sylvestris branches, 16 Aug. 2018, J.C. Zamora \& P. Posio (UPS F-941273). - NorWAY, Nord-Trøndelag, Meråker, on Pinus sylvestris branches, 24 July 2018, J.C. Zamora, M. Svensson \& M. Westberg (UPS F-941276). - RussIA, Karelia onegensis, Mdvezh' yegorsk regio, ad fonts fluminis Vitshka (UTM WQ3), on the roadside, hard, old wood, 21 June 1942, Matti Laurila (H 6020982).
- Sweden, Öland, Böda, Bödakusten västra naturreservat, Byrums Sandvik, $\mathrm{N} 57.236677^{\circ} \mathrm{E} 16.963497^{\circ}$, on Pinus sylvestris wood, 02 Oct. 2017, J.C. Zamora (UPS F-941268, UPS F-941269, duplicate in H); Södermanland, Stockholm, Skärholmen, N59.301129 ${ }^{\circ} \mathrm{E} 17.934630^{\circ}$, on Pinus sylvestris wood, 06 Apr. 2019, J.C. Zamora, P. Posio \& J. Nieminen (UPS F-941263); Värmland, Lekvattnet, N60.172306 ${ }^{\circ} \mathrm{E} 12.699782^{\circ}$, on Pinus sylvestris branch, 07 Oct. 2018, J.C. Zamora (UPS F-941267); Värmland, Ransäter, Munkfors V, N59.814609 ${ }^{\circ}$ E13.390181 ${ }^{\circ}$, on Pinus sylvestris branch, 08 June 2019, J.C. Zamora (UPS F-941467); Uppland, Älvkarleby, Långsandsörarna island, N60.65783 ${ }^{\circ}$ E17.55504 ${ }^{\circ}$, on Pinus sylvestris branches, 17 Apr. 2018, J.C. Zamora, S. Ekman \& M. Zuluaga (UPS F-941262); Uppland, Solna, Haga, coniferous wood, 01 July 2018, J.C. Zamora \& I. Olariaga (UPS F-941261); Uppland, Uppsala, Norra Lunsen, N59.80680 E17.69804 ${ }^{\circ}$, on old Pinus sylvestris wood, 20 Aug. 2017, J.C. Zamora (UPS F-941255, UPS F-914256); Uppland, Uppsala, Lunsen, on Pinus sylvestris branch, 08 Apr. 2018, J.C. Zamora (UPS F-941259); Uppland, Uppsala, Norra Lunsen, N59.790953 E17.687811 ${ }^{\circ}$, on Pinus sylvestris branch, 30 Sept. 2018, J.C. Zamora, S. Ekman, A. Kruys \& D. Pfister (UPS F-941260); Uppland, Uppsala, Stadsskogen naturreservat, $\mathrm{N} 59.84234^{\circ} \mathrm{E} 17.62527^{\circ}$, on highly decomposed, coniferous wood, 13 Aug. 2017, J.C. Zamora (UPS F-941254); Jämtland, Åre, Handöl, N63.245084 ${ }^{\circ} \mathrm{E} 12.444898^{\circ}$, on Pinus sylvestris branches, 24 July 2018, J.C. Zamora (UPS F-941265); Jämtland, Åre, N63.23271 ${ }^{\circ}$ E12.42884 ${ }^{\circ}$, on Pinus sylvestris wood, 25 July 2018, J.C. Zamora (UPS F-941266); Jämtland, Mörsil, Klukshåckren, N63.172614 ${ }^{\circ} \mathrm{E} 13.600665^{\circ}$, on Pinus sylvestris branches, 23 July 2018, J.C. Zamora (UPS F-941264, duplicate in H); Norrbotten, Övertorneå, Luppioberget, N66.313259 E23.608372 ${ }^{\circ}$, 13 Aug. 2019, J.C. Zamora \& P. Posio (UPS F-945010).

Unilacryma unispora (L.S. Olive) Shirouzu, Tokum. \& Oberw., Mycologia 105: 1120. 2013 s.lat. — Fig. 15

इ Platygloea unispora L.S. Olive, J. Elisha Mitchell Sci. Soc. 74: 41. 1958 [basion.].

- Achroomyces unisporus (L.S. Olive) Wojewoda, Mala FI. Grzybow 2: 205. 1981, nom. inval. (Art. 6.10, Art. 41.8 does not apply).

- Dacrymyces unisporus (L.S. Olive) K. Wells, Mycologia 86: 31. 1994, nom. inval. (see above).

Typus. United States of America, North Carolina, Orange, [Chapel Hill], Battle Park, 11 Dec. 1943, L.S. Olive s.n. (NCU-F-0026842! holotype).

Basidiocarps \pm gelatinous, amber-coloured to brownish, xerotolerant, isolated, gregarious or crowded, initially erumpent, pulvinate, tardily becoming depressed-pulvinate, without a conspicuous margin, 0.3-1.0(-1.2) $\mathrm{mm}$ diam. Cortical/marginal hyphae slightly to clearly thick-walled, with cylindrical or attenuate terminal cells, 4-7 $\mu \mathrm{m}$ wide, unbranched or rarely sparingly branched, with sparse anastomoses and both clamped and clampless septa. Internal hyphae 3-7 $\mu \mathrm{m}$ wide, slightly to clearly thick-walled, embedded in a gelatinous matrix, with clamp connections but also frequently with some simple septa. Hymenium almost amphigenous, progressively forming numerous basidia intermixed with some hyphidia. Subhymenium with thin to slightly thick-walled, clamped hyphae. Hyphidia hyaline, sparsely to heavily branched, $(2.5-) 3-4.5 \mu \mathrm{m}$ thick in the upper half, terminal branches (1-)1.5-3 $\mu \mathrm{m}$ thick, often with clamped septa throughout their length. Young basidia initials cylindric to narrowly claviform, 5-7.5 $\mu \mathrm{m}$ thick when close to maturity. Mature basidia developing a single thick sterigma (very rarely two irregularly formed sterigma-like structures may be seen), about 18-32 $\mu \mathrm{m}$ long, bearing a single terminal and asymmetrically attached basidiospore. Total basidium length reaching 70-130 $\mu \mathrm{m}$. Basidiospores (11.9-)14-17.4(-19.5) $\times$ (10.2-)11.2-13.5(-15.7) $\mu \mathrm{m}, \mathrm{Q}=(1.1-) 1.2-1.4(-1.6)$, ovoid to broadly ellipsoid, rarely globose or ellipsoid, with a conspicuous 1.0-1.5 $\mu \mathrm{m}$ long hilar appendix, becoming transversely 1-septate and developing additional oblique, longitudinal and transverse septa, then becoming muriform, with a single hardly visible nucleus when still aseptate. Basidium and basidiospore cytoplasm hyaline or with a very pale yellowish tinge, with numerous small lipid bodies that slowly coalesce in dead state to form conspicuous lipid drops. Basidiospores germinating by the production of hyphae or microconidia. Microconidia rarely 


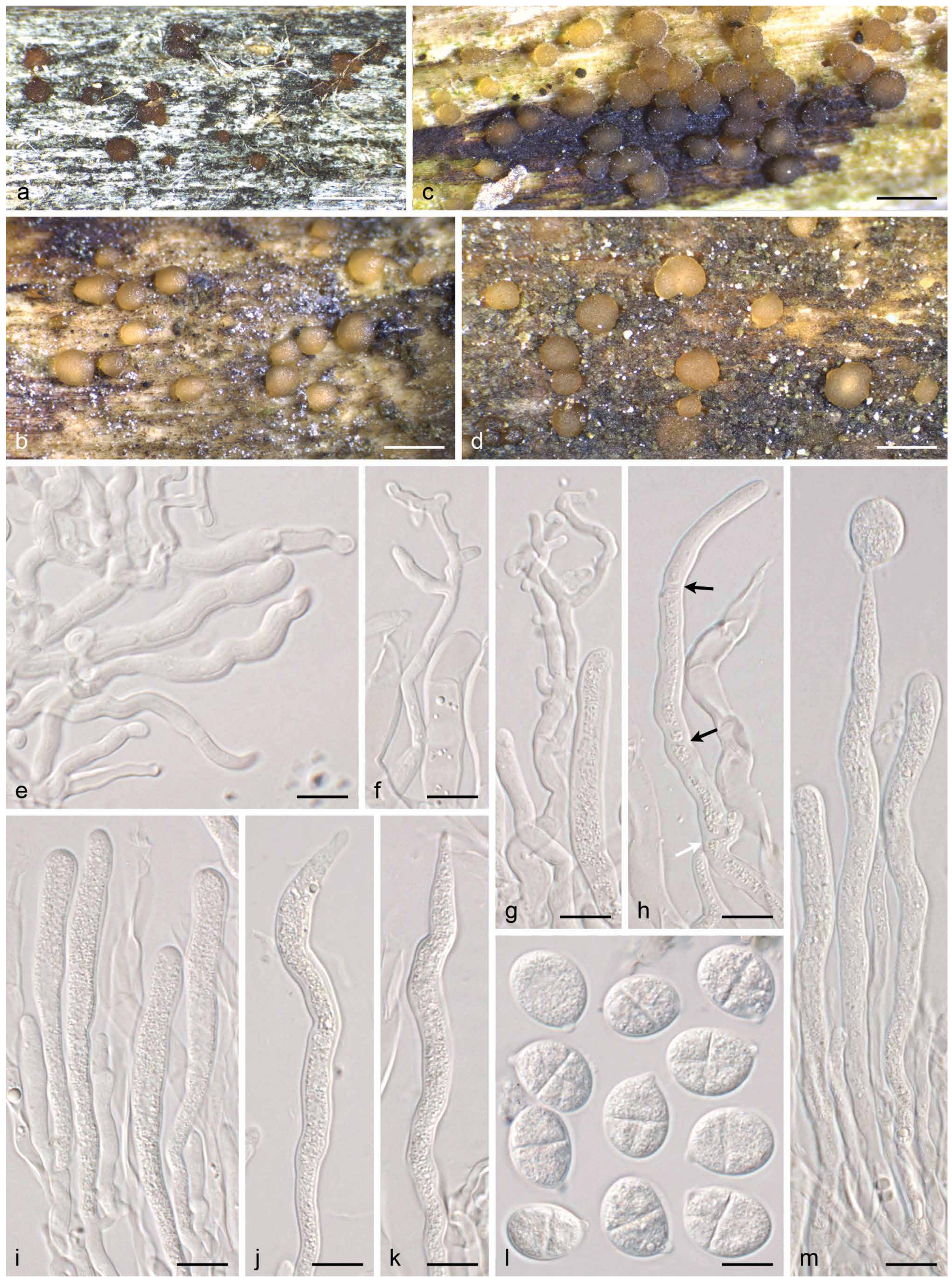

Fig. 15 Unilacryma unispora s.lat. a-d. Fruitbodies in different degrees of development, all in fresh condition except a; e-m. Micromorphology; e. marginal hyphae; $f-g$. branched hyphidia; $h$. terminal cells of marginal hyphae, note the presence of both clampless (black arrows) and clamped (white arrow) septa; i. young basidia; $j-k$. mature basidia; I. basidiospores; $m$. two young basidia and one mature basidium bearing a single sterigma with a terminal basidiospore. a. From the holotype; $b, e-g, j-k$. from UPS F-941279; c. from UPS F-941286; d. from UPS F-941284; $h-i$, m. from UPS F-941283; I. from UPS F-941280. - Scale bars: $a-d=1 \mathrm{~mm}, \mathrm{e}-\mathrm{m}=10 \mu \mathrm{m}$. 
observed, narrowly ellipsoid to cylindric, about 3-4 × $1.5 \mu \mathrm{m}$, hyaline.

Ecology \& Distribution - Very common, frequently growing on decorticated, old and rather exposed wood of Juniperus spp. and Pinus spp., and particularly common on dead branches still attached to trees. In areas where the distributions of both Unilacryma species overlap, they may grow together on the same branch and even intermixed. In Europe, U. unispora has been previously reported only from The Netherlands by Dam \& Dam (2014). In a wide sense, it is broadly distributed in the Holarctic temperate biome (North America, East Asia, and Europe), and extends into the southern boreal zone, but it has not been found in the middle and northern boreal forests of Sweden and Finland where $U$. bispora is common.

Nomenclature \& Taxonomy - When describing Platygloea unispora, Olive (1944) failed to include the required Latin diagnosis, resulting in an invalid name (ICN, Art. 39.1). The taxon was validated by Olive (1958), who provided the required diagnosis as well as a holotype. Olive (1944) is often incorrectly cited as the place of publication of the basionym, which renders the intended new combinations in Achroomyces and Dacrymyces invalid, none of the requirements of Art. 41.8 being fulfilled.

In a broad taxonomic sense, $U$. unispora is well distinguished by 1 -spored basidia and subglobose to ovoid basidiospores that become transversely septate and finally muriform with age. The macromorphology is nearly identical to that of $U$. bispora, so a micromorphological study of the samples is always needed to identify them. The European specimens agree morphologically very well with the description of Olive (1944) and additional descriptions and iconography of North American specimens (e.g., Wells 1994), as well as with the North American specimens we studied, including the holotype. Unfortunately, we were not able to obtain DNA sequence data of the studied North American specimens, not even after using specific primers to amplify short fragments of the ITS. The description of the Japanese specimens by Shirouzu et al. (2009) also agrees relatively well, although they reported basidia and basidiospores to be on average slightly smaller, marginal hyphae as thin-walled, and branched hyphidia as absent. In addition, the samples from North Europe and East Asia form two different clades (Fig. 10). Nevertheless, with the current morphological and genetic data, it is unclear whether these clades represent two distinct species. Therefore, we assume here a conservative approach and treat the North American, East Asian, and European specimens as different populations of a single species.

Additional specimens examined. FInLAND, Alandia, Kökar, Hamno, N59.947100 ${ }^{\circ} \mathrm{E} 20.875280^{\circ}$, on Juniperus communis branch, 22 July 2019, J.C. Zamora \& P. Posio (UPS F-945056); Regio aboensis, Nauvo, N60.192401 ${ }^{\circ}$ E21.912373 ${ }^{\circ}$, on Pinus sylvestris branch, 21 July 2019, J.C. Zamora \& P. Posio (UPS F-945073); Nylandia, Espoo, near Nuuksio National park, on Pinus sylvestris branch, 12 May 2018, J.C. Zamora \& P. Posio (UPS F-941295); Nylandia, Helsinki, Veräjämäki, old, rather moist forest depression, on a fallen branch (Pinus sylvestris, decay stage 3, $3 \mathrm{~cm}$ diam), N60.22390 E24.97607 ${ }^{\circ}$, elev. $29 \mathrm{~m}, 25$ May 2010, O. Miettinen 14064 (H 6012675); Tavastia australis, Tammela, near Saaren Kansapuisto, on Pinus sylvestris branch, 11 May 2018, J.C. Zamora \& P. Posio (UPS F-941298); Tavastia australis, Tammela, Mustiala, N60.816501 ${ }^{\circ}$ E23.764646 ${ }^{\circ}$, on Pinus sylvestris branches, 11 May 2018, J.C. Zamora \& P. Posio (UPS F-941296); Tavastia australis, Tammela, Mustiala, Salois (Saloinen), around P.A. Karsten's house, on Pinus sylvestris branch, 11 May 2018, J.C. Zamora \& P. Posio (UPS F-941297). - NorwAY, Hedmark, Kongsvinger, Austmarka, N60.106530 ${ }^{\circ} 12.358369^{\circ}$, on Pinus sylvestris branch, 06 Oct. 2018, J.C. Zamora \& P. Posio (UPS F-941293); Hedmark, Kongsvinger, Kubergskogen, N60.236862 ${ }^{\circ} \mathrm{E} 12.426987^{\circ}$, on Pinus sylvestris branch, 06 Oct. 2018, J.C. Zamora \& P. Posio (UPS F-941294); Troms, Storfjord, Skibotn, N69.389745 ${ }^{\circ}$ E20.296217 ${ }^{\circ}$, on Pinus sylvestris wood, 16 Aug. 2019, J.C. Zamora \& P. Posio (UPS F-945011). - Sweden, Öland, Böda, Bödakusten västra naturreservat, Byrums Sandvik, N57.236677 ${ }^{\circ} \mathrm{E} 16.963497^{\circ}$, on Pinus sylvestris branches, 02 Oct. 2017, J.C. Zamora (UPS F-941291, duplicate in H);
Öland, Böda, Lindreservatet, N57.319448 ${ }^{\circ}$ E17.030849 ${ }^{\circ}$, on Pinus sylvestris wood, 03 Oct. 2017, J.C. Zamora (UPS F-941292); Södermanland, Nacka, Nackareservatet, N59.28955 ${ }^{\circ} 18.17560^{\circ}$, on Pinus sylvestris branches, 10 June 2018, J.C. Zamora, P. Posio \& J. Nieminen (UPS F-941288); Värmland, Lekvattnet, N60.172306 ${ }^{\circ}$ E12.699782 ${ }^{\circ}$, on Pinus sylvestris branch, 07 Oct. 2018, J.C. Zamora (UPS F-941290); Värmland, Ransäter, Munkfors V, N59.814677 ${ }^{\circ}$ E13.391138 , on Pinus sylvestris branches, 08 June 2019, J.C. Zamora (UPS F-941466); Uppland, Älvkarleby, Långsandsörarna island, N60.65783 ${ }^{\circ} \mathrm{E} 17.55504^{\circ}$, on Pinus sylvestris branches, 17 Apr. 2018, J.C. Zamora, S. Ekman \& M. Zuluaga (UPS F-941283); Uppland, Funbo, c. $400 \mathrm{~m}$ north of Fjällnora farm, N59.840762 ${ }^{\circ}$ E17.913327 ${ }^{\circ}$, on Pinus sylvestris branch, 14 Nov. 2017, J.C. Zamora \& S. Ekman (UPS F-941284); Uppland, Jumkil, Granslätt, N59.95312 ${ }^{\circ} \mathrm{E} 17.27913^{\circ}$, on old pieces of a rabbit cage, made of unidentified (presumably coniferous) wood, 12 May 2017, J.C. Zamora, S. Ekman \& M. Westberg (UPS F-941277); Uppland, Solna, Bergshamra, N59.38358 $\mathrm{E} 18.03756^{\circ}$, on Pinus sylvestris branch, 23 June 2018, J.C. Zamora (UPS F-941286); Uppland, Solna, Hagaparken, N59.365065 ${ }^{\circ} 18.032583^{\circ}$, on unidentified weathered wood, probably Pinus sylvestris, 28 Apr. 2019, J.C. Zamora (UPS F-941287); Uppland, Uppsala, Norra Lunsen, N59.80680 E17.69804 ${ }^{\circ}$, on Pinus sylvestris branches, 20 Aug. 2017, J.C. Zamora (UPS F-941279, duplicate in H); Uppland, Uppsala, Norra Lunsen naturreservat, N59.79390 E17.68667 ${ }^{\circ}$, on Pinus sylvestris branches, 19 Nov. 2017, J.C. Zamora (UPS F-941280); Uppland, Uppsala, Norra Lunsen naturreservat, on Pinus sylvestris branches, 08 Apr. 2018, J.C. Zamora (UPS F-941281); Uppland, Uppsala, Norra Lunsen naturreservat, N59.790953 ${ }^{\circ}$ E16.687811 ${ }^{\circ}$, on Pinus sylvestris branches, 30 Sept. 2018, J.C. Zamora (UPS F-941282); Uppland, Uppsala, Stadsskogen naturreservat, N59.84234 $4^{\circ} 17.62527^{\circ}$, on highly decomposed Pinus sylvestris wood, 13 Aug. 2017, J.C. Zamora (UPS F-941278); Hälsingland, Gnarp, N62.037699 E17.154672 ${ }^{\circ}$, on exposed Pinus sylvestris branch, 27 July 2018, J.C. Zamora (UPS F-941289). - UnITEd States of AmerICA, North Carolina, Orange, University Lake area, near Chapel Hill, Juniperus virginiana, 14 Apr. 1982, L.S. Olive NC82-40 (NCU F-0026843).

Acknowledgements We are grateful to G. Bonito, P. Crous, D. Eastwood, J. Magnuson, F. Martin, S. Maurice, O. Miettinen, L. Nagy, E. Record, J. Spatafora, and P. Wang, from the 1000 Fungal Genomes Consortium, for providing access to unpublished genome data. Those genome sequence data were produced by the US Department of Energy Joint Genome Institute in collaboration with the user community. B. Matheny kindly gave permission to use an unpublished sequence of Calocera viscosa from the AFTOL database. Metsähallitus (Finland) is thanked for providing permission for collecting Dacrymycetes samples in state-owned forests of Finland. We also acknowledge M. Svensson, M. Westberg, and P. Posio for their help with sampling specimens, as well as O. Miettinen, M. Svensson, A. Savchenko, and three anonymous reviewers for useful comments that improved the text. This work was supported by the project 'Taxonomic study of the Nordic Dacrymycetes' financed by the Swedish Taxonomy Initiative (Svenska artprojektet, grant no 2016-28 4.3) and the José Castillejo programme from the Spanish government (CAS16/00063).

\section{REFERENCES}

Arpin N, Fiasson J-L. 1971. The pigments of Basidiomycetes: their chemotaxonomic interest. In: Petersen RH (ed), Evolution in the higher basidiomycetes: 63-98. University of Tennessee Press, USA.

Avalos J, Limón MC. 2015. Biological roles of fungal carotenoids. Current Genetics 61: 309-324.

Avise JC, Johns GC. 1999. Proposal for a standardized temporal scheme of biological classification for extant species. Proceedings of the National Academy of Sciences of the United States of America 96: 7358-7363.

Bandoni RJ. 1963. Dacrymyces ovisporus from British Columbia. Mycologia 55: $360-361$

Baral HO. 1992. Vital versus herbarium taxonomy: morphological differences between living and dead cells of ascomycetes, and their taxonomic implications. Mycotaxon 44: 333-390.

Baral HO. 2004. The European and North-American species of Sarcoscypha. http://www.gbif-mycology.de/HostedSites/Baral/Sarcoscypha.htm.

Beaulieu JM, O'Meara BC, Donoghue MJ. 2013. Identifying hidden rate changes in the evolution of a binary morphological character: The evolution of plant habit in campanulid angiosperms. Systematic Biology 62: 725-737. Bodman MC. 1938. Morphology and cytology of Guepinia spathularia. Mycologia 30: 635-652.

Boidin J, Gilles G. 1986. Basidiomycètes Aphyllophorales de l'île de la Réunion. VI. Le genere Cerinomyces Martin. Bulletin trimestriel de la Société mycologique de France 102: 315-319. 
Bollback JP. 2006. SIMMAP: Stochastic character mapping of discrete traits on phylogenies. BMC Bioinformatics 7: 88 .

Borchsenius F. 2009. FastGap 1.2. Department of Biosciences, Aarhus University, Denmark. Published online at http://www.aubot.dk/FastGap_home. htm.

Bouckaert RR, Drummond AJ. 2017. bModelTest: Bayesian phylogenetic site model averaging and model comparison. BMC Evolutionary Biology 17: 42. Bouckaert RR, Heled J, Kühnert D, et al. 2014. BEAST 2: A software platform for Bayesian evolutionary analysis. PLoS Computational Biology: 10: e1003537.

Brasfield TW. 1938. The Dacrymycetaceae of temperate North America. The American Midland Naturalist 20: 211-235.

Brefeld O. 1888. Untersuchungen aus dem Gesammtgebiete der Mykologie 7. Felix, Germany.

Brough S, Bandoni R. 1975. Epistolae mycologicae. VI. Occurrence of Dacryonaema in British Columbia. Syesis 8: 301-303.

Cederberg E, Neujahr HY. 1970. Distribution of $\beta$-carotene in subcellular fractions of Blakeslea trispora. Experientia 26: 366-367.

Chambers EA, Hillis DM. 2019. The multispecies coalescent over-splits species in the case of geographically widespread taxa. Systematic Biology syz042. doi: https://doi.org/10.1093/sysbio/syz042.

Christenhusz MJM, Vorontsova MS, Fay MF, et al. 2015. Results from an online survey of family delimitation in angiosperms and ferns: recommendations to the Angiosperm Phylogeny Group for thorny problems in plant classification. Botanical Journal of the Linnnean Society 178: 501-528.

Clémençon H. 2004. Cytology and plectology of the hymenomycetes. Bibliotheca Mycologica 199: 1-488.

Crotty SM, Minh BQ, Bean NG, et al. 2017. GHOST: Recovering historical signal from heterotachously-evolved sequence alignments. Available at bioRxiv. doi: https://doi.org/10.1101/174789.

Cubeta MA, Echandi E, Abernethy T, et al. 1991. Characterization of anastomosis groups of binucleate Rhizoctonia species using restriction analysis of an amplified ribosomal RNA gene. Phytopathology 81: 1395-1400.

Cusimano N, Renner SS. 2014. Ultrametric trees or phylograms for ancestral state reconstruction: Does it matter? Taxon 63: 721-726.

Czeczuga B. 1980. Badania nad karotenoidami u grzybów. IX. Dacrymycetaceae. Acta Mycologica 16: 115-120.

Dam N, Dam M. 2014. A Dacrymyces with 1-spored basidia. Coolia 57: 215217.

Dangeard PA. 1895. Memoire sur la reproduction sexuelle des Basidiomycetes. Le Botaniste 4: 119-181.

Degnan JH, Rosenberg NA. 2009. Gene tree discordance, phylogenetic inference and the multispecies coalescent. Trends in Ecology \& Evolution 24: 332-340.

Donk MA. 1966. Check list of European hymenomycetous Heterobasidiae. Persoonia 4: 145-335.

Dorsey BL, Gregory TJ, Sass C, et al. 2018. Pleistocene diversification in an ancient lineage: a role for glacial cycles in the evolutionary history of Dioon Lindl. Zamiaceae. American Journal of Botany 105: 1512-1530.

Drummond AJ, Ho SYW, Phillips MJ, et al. 2006. Relaxed phylogenetics and dating with confidence. PLoS Biology 4: e88.

Duncan EG, Chisholm JRM. 1982. Post-meiotic events in Dacrymyces stillatus. Transactions of the British Mycological Society 79: 299-303.

Duncan EG, Galbraith MH. 1972. Post-meiotic events in the Homobasidiomycetidae. Transactions of the British Mycological Society 58: 387-392.

Echavarri-Erasun C, Johnson EA. 2002. Fungal carotenoids. Applied Mycology and Biotechnology 2: 45-85.

Eckblad F-E. 1968. The genera of operculate discomycetes. A re-evaluation of their taxonomy, phylogeny and nomenclature. Nytt magasin for botanikk 15: 1-191.

Ferencova Z, Rico VJ, Hawksworth DL. 2017. Extraction of DNA from lichenforming and lichenicolous fungi: A low-cost fast protocol using Chelex. The Lichenologist 49: 521-525.

Fernández-Mazuecos M, Mellers G, Vigalondo B, et al. 2018. Resolving recent plant radiations: power and robustness of genotyping-by-sequencing. Systematic Biology 67: 250-268.

Fiasson JL. 1973. The carotenoids of some Basidiomycetes. NASA Technical Translation F-14: 858.

FitzJohn RG, Maddison WP, Otto SP. 2009. Estimating trait dependent speciation and extinction rates from incompletely resolved phylogenies. Systematic Biology 58: 595-611.

Floudas D, Binder M, Riley R, et al. 2012. The Paleozoic origin of enzymatic lignin decomposition reconstructed from 31 fungal genomes. Science 336: $1715-1719$.

Fries EM. 1818. Uppställning af de i Sverige funne Vårtsvampar. Scleromyci. Kongliga Vetenskaps Academiens handlingar för år 1818 [ser. 3, vol. 6]: 356-361.
Fries EM. 1823. Systema Mycologicum 2. Ex officina Berlingiana, Sweden. Gardes M, Bruns TD. 1993. ITS primers with enhanced specificity for basidiomycetes. Application to the identification of mycorrhizae and rusts. Molecular Ecology 2: 113-118.

Gargas A, Taylor JW. 1992. Polymerase chain reaction. PCR primers for amplifying and sequencing nuclear $18 \mathrm{~S}$ rDNA from lichenized fungi. Mycologia 84: 589-592.

Garnica S, Riess K, Schön ME, et al. 2016. Divergence times and phylogenetic patterns of Sebacinales, a highly diverse and widespread fungal lineage. PLoS ONE 11: e0149531. doi: https://doi.org/10.1371/journal. pone.0149531.

Gill M, Steglich W. 1987. Pigments of fungi. Macromycetes. Fortschritte der Chemie organischer Naturstoffe 51: 1-317.

Goldstrohm DD, Lilly VG. 1965. The effect of light on the survival of pigmented and nonpigmented cells of Dacryopinax spathularia. Mycologia 57: $612-623$

Goodwin TW. 1953. Studies in carotenogenesis 8. The carotenoids present in the basidiomycete Dacryomyces stillatus. Biochemical Journal 53: $538-540$.

Griffin DH. 1994. Fungal physiology. Wiley-Liss, New York.

Grigoriev IV, Nikitin R, Haridas S, et al. 2014. MycoCosm portal: gearing up for 1000 fungal genomes. Nucleic Acids Research 42: D699-704.

Guindon S, Dufayard J-F, Lefort V, et al. 2010. New algorithms and methods to estimate maximum-likelihood phylogenies: assessing the performance of PhyML 3.0. Systematic Biology 59: 307-321.

Hanna C, Bulat TJ. 1953. Pigment study of Dacrymyces ellisii. Mycologia 45: $143-144$

Hansen K, Perry BA, Dranginis AW, et al. 2013. A phylogeny of the highly diverse cup-fungus family Pyronemataceae. Pezizomycetes, Ascomycota clarifies relationships and evolution of selected life history traits. Molecular Phylogenetics and Evolution 67: 311-335.

He M-Q, Zhao R-L, Hyde KD, et al. 2019. Notes, outline and divergence times of Basidiomycota. Fungal Diversity 99: 105-367.

Heled J, Drummond AJ. 2010. Bayesian inference of species trees from multilocus data. Molecular Biology and Evolution 27: 570-580.

Henriot A, Cheype J-L. 2016. Piximètre. http://piximetre.fr/ [accessed 01 June 2018].

Hibbett DS, Binder M, Bischoff JF, et al. 2007. A higher-level phylogenetic classification of the Fungi. Mycological Research 111: 509-547.

Holt BG, Jønsson KA. 2014. Reconciling hierarchical taxonomy with molecular phylogenies. Systematic Biology 63: 1010-1017.

Huelsenbeck JP, Larget B, Alfaro MA. 2004. Bayesian phylogenetic model selection using reversible jump Markov chain Monte Carlo. Molecular Biology and Evolution 21: 1123-1133.

Istvanffi G. 1895. Uber die Rolle der Zellkerne bei der Entwicklung der Pilze. Berichte der Deutschen Botanischen Gesellschaft 13: 452-467.

Jones G. 2017. Algorithmic improvements to species delimitation and phylogeny estimation under the multispecies coalescent. Journal of Mathematical Biology 74: 447-467.

Jones G, Aydin Z, Oxelman B. 2014 '2015'. DISSECT: an assignment-free Bayesian discovery method for species delimitation under the multispecies coalescent. Bioinformatics 31: 991-998.

Juel HO. 1898. Die Kerntheilung in d. Basidien und die Phylogenie der Basidiomyceten. Jahrbücher für Wissenschaftliche Botanik 32: 361-388.

Junier T, Zdobnov EM. 2010. The newick utilities: high-throughput phylogenetic tree processing in the UNIX Shell. Bioinformatics 26: 1669-1670.

Kalyaanamoorthy S, Minh BQ, Wong TKF, et al. 2017. ModelFinder: fast model selection for accurate phylogenetic estimates. Nature Methods 14: $587-589$.

Keller A, Schleicher T, Schultz J, et al. 2009. 5.8S-28S rRNA interaction and HMM-based ITS2 annotation. Gene 430: 50-57.

Kennedy LL. 1959a '1958'. The genera of the Dacrymycetaceae. Mycologia 50: $874-895$

Kennedy LL. 1959b '1958'. The genus Dacrymyces. Mycologia 50: 896-915. Kirk PM, Cannon PF, Minter DW, et al. 2008. Dictionary of the fungi, 10th ed. CABI Europe, UK.

Kirschner R, Yang ZL. 2006 '2005'. Dacryoscyphus chrysochilus, a new staurosporous anamorph with cupulate conidiomata from China and with affinities to the Dacrymycetales. Basidiomycota. Antonie Van Leeuwenhoek 87: $329-337$

Kistenich S, Timdal E, Bendiksby M, et al. 2018. Molecular systematics and character evolution in the lichen family Ramalinaceae. Ascomycota: Lecanorales. Taxon 67: 871-904.

Kobayasi Y. 1939a. On the Dacrymyces group. Science Reports of the Tokyo Bunrika Daigaku B 4: 105-128.

Kobayasi Y. 1939b. On the genera Femsjonia, Guepinia and Calocera from Japan. Science Reports of the Tokyo Bunrika Daigaku B 4: 215-227. 
Kohler A, Kuo A, Nagy LG, et al. 2015. Convergent losses of decay mechanisms and rapid turnover of symbiosis genes in mycorrhizal mutualists. Nature Genetics 47: 410-415.

Kosakovsky Pond SL, Posada D, Gravenor MB, et al. 2006. Automated phylogenetic detection of recombination using a genetic algorithm. Molecular Biology and Evolution 23: 1891-1901.

Kourist R, Bracharz F, Lorenzen J, et al. 2015. Genomics and transcriptomics analyses of the oil-accumulating basidiomycete yeast Trichosporon oleaginosus: insights into substrate utilization and alternative evolutionary trajectories of fungal mating systems. mBio 6: e00918.

Kraichak E, Huang J-P, Nelsen M, et al. 2018. A revised classification of orders and families in the two major subclasses of Lecanoromycetes. Ascomycota based on a temporal approach. Botanical Journal of the Linnean Society 188: 233-249.

Kretzer A, Bruns TD. 1999. Use of atp6 in fungal phylogenetics: an example from the Boletales. Molecular Phylogenetics and Evolution 13: 483-492.

Kubart A, Vasaitis R, Stenlid J, et al. 2016. Fungal communities in Norway spruce stumps along a latitudinal gradient in Sweden. Forest Ecology and Management 371: 50-58.

Kubatko LS, Degnan JH. 2007. Inconsistency of phylogenetic estimates from concatenated data under coalescence. Systematic Biology 56: 17-24.

Lange M. 1955. Systematisk Botanik bind II, nr. 1. Svampe. Munksgaard, Denmark.

Læssøe T, Petersen JH. 2014. Mycokey 4.1, the fungus information system. http://www.mycokey.com/. [accessed 01 Oct. 2018].

Litsios G, Salamin N. 2012. Effects of phylogenetic signal on ancestral state reconstruction. Systematic Biology 61: 533-538.

Liu YJ, Whelen S, Hall BD. 1999. Phylogenetic relationships among ascomycetes: evidence from an RNA polymerse II subunit. Molecular Biology and Evolution 16: 1799-1808.

Lopez P, Casane D, Philippe H. 2002. Heterotachy, an important process of protein evolution. Molecular Biology and Evolution 19: 1-7.

Lücking R. 2019. Stop the abuse of time! Strict temporal banding is not the future of rank-based classifications in Fungi (including lichens) and other organisms. Critical Reviews in Plant Sciences 38: 199-253.

Lüdecke D. 2019. sjstats: Statistical functions for regression models, v. 0.17.4. doi: https://doi.org/10.5281/zenodo.1284472, https://CRAN.R-project.org/ package=sjstats. [accessed 01 Oct. 2018].

Maddison WP, Midford PE, Otto SP. 2007. Estimating a binary character's effect on speciation and extinction. Systematic Biology 56: 701-710.

Maekawa N. 1987. A new species of the genus Cerinomyces. Canadian Journal of Botany 65: 583-588.

Maire R. 1902. Recherches cytologiques et taxonomiques sur les Basidiomycetes. Bulletin de la Société Mycologique de France 18 (supplement).

Malençon G, Bertault H. 1971. Champignons de la Péninsule Ibérique. Acta Phytotaxonomica Barcinonensia 8: 1-64.

Martin GW. 1949. The genus Ceracea Cragin. Mycologia 41: 77-86.

Martin GW. 1952. Revision of the north central Tremellales. State University of lowa Studies in Natural History 19: 1-122.

Mason-Gamer RJ, Kellogg EA. 1996. Testing for phylogenetic conflict among molecular data sets in the tribe Triticeae. Gramineae. Systematic Biology 45: 524-545.

Matheny PB. 2005. Improving phylogenetic inference of mushrooms with RPB1 and RPB2 nucleotide sequences. Inocybe; Agaricales. Molecular Phylogenetics and Evolution 35: 1-20.

Matheny PB, Liu YJ, Ammirati JF, et al. 2002. Using RPB1 sequences to improve phylogenetic inference among mushrooms. Inocybe, Agaricales. American Journal of Botany 89: 688-698.

Mathiesen H. 1991. The genus Dacrymyces in Denmark. Svampe 23: 46-61.

McLaughlin DJ, Healy RA, Arun Kumar TK, et al. 2016. Cultural and cytological characterization of Dacryopinax primogenitus, a new species in the Dacrymycetes with a fully sequenced genome. Mycologia 108: 457-468.

McNabb RFR. 1964. Taxonomic studies in the Dacrymycetaceae I. Cerinomyces Martin. New Zealand Journal of Botany 2: 415-424.

McNabb RFR. 1965a. Taxonomic studies in the Dacrymycetaceae II. Calocera Fries. New Zealand Journal of Botany 3: 31-58.

McNabb RFR. 1965b. Taxonomic studies in the Dacrymycetaceae III. Dacryopinax Martin. New Zealand Journal of Botany 3: 59-72.

McNabb RFR. 1965c. Taxonomic studies in the Dacrymycetaceae IV. Guepiniopsis Patouillard. New Zealand Journal of Botany 3: 159-169.

McNabb RFR. 1965d. Taxonomic studies in the Dacrymycetaceae V. Heterotextus Lloyd. New Zealand Journal of Botany 3: 215-222.

McNabb RFR. 1965e. Taxonomic studies in the Dacrymycetaceae VI. Femsjonia Fries. New Zealand Journal of Botany 3: 223-228.

McNabb RFR. 1966. Taxonomic studies in the Dacrymycetaceae VII. Ditiola Fries. New Zealand Journal of Botany 4: 546-558.

McNabb RFR. 1973. Taxonomic studies in the Dacrymycetaceae VIII. Dacrymyces Nees ex Fries. New Zealand Journal of Botany 11: 461-524.
Miller MA, Pfeiffer W, Schwartz T. 2010. Creating the CIPRES Science Gateway for 641 inference of large phylogenetic trees. Proc. Gateway Computing Environments Workshop 642. GCE, 14 Nov. 2010, New Orleans, LA, 1-8. doi: https://doi.org/10.1109/GCE.2010.5676129.

Mirarab S, Nguyen N, Guo S, et al. 2015. PASTA: Ultra-large multiple sequence alignment for nucleotide and aminoacid sequences. Journal of Computational Biology 22: 377-386.

doi: https://doi.org/10.1089/cmb.2014.0156.

Moliné M, Flores MR, Libkind D, et al. 2010. Photoprotection by carotenoid pigments in the yeast Rhodotorula mucilaginosa: The role of torularhodin. Photochemical and Photobiological Sciences 9: 1145-1151.

Morin E, Kohler A, Baker AR, et al. 2012. Genome sequence of the button mushroom Agaricus bisporus reveals mechanisms governing adaptation to a humic-rich ecological niche. Proceedings of the National Academy of Sciences of the United States of America 109: 17501-17506.

Nagy LG, Riley R, Tritt A, et al. 2015 '2016'. Comparative genomics of earlydiverging mushroom-forming fungi provides insights into the origins of lignocellulose decay capabilities. Molecular Biology and Evolution 33: 959-970.

Nannfeldt JA. 1947. Sphaeronema rufum Fr., a misunderstood member of Dacrymycetaceae. Svensk Botanisk Tidskrift 41: 321-339.

Neuhoff W. 1936. Die Gallertpilze Schwedens: Tremellaceae, Dacrymycetaceae, Tulasnellaceae, Auriculariaceae. Arkiv för Botanik 28A: 1-57.

Nguyen L-T, Schmidt HA, Von Haeseler A, et al. 2015. IQ-TREE: A fast and effective stochastic algorithm for estimating maximum likelihood phylogenies. Molecular Biology and Evolution 32: 268-274. doi: https://doi. org/10.1093/molbev/msu300.

Oberwinkler F. 1989. Ditiola haasii sp. nov., a new species in the Dacrymycetales. Zeitschrift für Mykologie 55: 197-206.

Oberwinkler F. 1994. Genera in a monophyletic group: the Dacrymycetales. Mycologia Helvetica 6: 35-72.

Oberwinkler F. 2014. Dacrymycetes. In: McLaughlin DJ, Spatafora JW (eds), The Mycota VII. Systematics and Evolution, part A: 357-372. SpringerVerlag, Germany.

Oberwinkler F, Tschen J. 1989. A new Dacrymyces species from Taiwan. Transactions of the Mycological Society of Japan 30: 349-356.

Ogilvie HA, Bouckaert RR, Drummond AJ. 2017. StarBEAST2 brings faster species tree inference and accurate estimates of substitution rates. Molecular Biology and Evolution 34: 2101-2114.

Ogilvie HA, Heled J, Xie D, et al. 2016. Computational performance and statistical accuracy of ${ }^{*} \mathrm{BEAST}$ and comparisons with other methods. Systematic Biology 65: 381-396.

Olariaga I, Buyck B, Esteve-Raventós F, et al. 2015. Assessing the taxonomic identity of white and orange specimens of Cantharellus: occasional colour variants or independent species? Cryptogamie, Mycologie 36: 287-301.

Olive LS. 1944. New or rare heterobasidiomycetes from North Carolina - I. Journal of the Elisha Mitchell Scientific Society 60: 17-26.

Olive LS. 1958. Latin diagnoses of earlier described species of Tremellales. Journal of the Elisha Mitchell Scientific Society 74: 41.

Pamilo P, Nei M. 1988. Relationships between gene trees and species trees. Molecular Biology and Evolution 5: 568-583.

Paradis E. 2013. Molecular dating of phylogenies by likelihood methods: A comparison of models and a new information criterion. Molecular Phylogenetics and Evolution 67: 436-444.

Paradis E, Schliep K. 2019. ape 5.0: an environment for modern phylogenetics and evolutionary analyses in R. Bioinformatics 35: 526-528.

Paraskevis D, Deforche K, Lemey P, et al. 2005. SlidingBayes: exploring recombination using a sliding window approach based on Bayesian phylogenetic inference. Bioinformatics 21: 1274-1275.

Pilz D, Norvell L, Danell E, et al. 2003. Ecology and management of commercially harvested chanterelle mushrooms. Pacific Northwest Research Station. General technical report. USA.

R Core Team. 2018. R: A language and environment for statistical computing. R Foundation for Statistical Computing, Vienna. https://www.R-project.org [accessed 01 Oct. 2018].

Rabiee M, Sayyari E, Mirarab S. 2019. Multi-allele species reconstruction using ASTRAL. Molecular Phylogenetics and Evolution 130: 286-296.

Raitviir AG. 1967. Key to heterobasidiomycetidae of the U.S.S.R. Nauka, Leningrad.

Rambaut A. 2016. Molecular evolution, phylogenetics and epidemiology. FigTree v1.4. http://tree.bio.ed.ac.uk/software/figtree/ [accessed 01 Feb. 2017].

Rambaut A, Drummond AJ, Xie D, et al. 2018. Posterior summarisation in Bayesian phylogenetics using Tracer 1.7. Systematic Biology 67: 901-904. doi: https://doi.org/10.1093/sysbio/syy032.

Redhead S. 1997. Macrofungi of British Columbia; requirements for inventory. Research Branch, British Columbia Ministry of Forests, and Wildlife Branch, British Columbia Ministry of Environment, Lands and Parks, Victoria, British Columbia. Working Paper 28/1997. USA. 
Rehner SA, Buckley E. 2005. A Beauveria phylogeny inferred from nuclear ITS and EF1- $\alpha$ sequences: evidence for cryptic diversification and links to Cordyceps teleomorphs. Mycologia 97: 84-98.

Reid DA. 1974. A monograph of the British Dacrymycetales. Transactions of the British Mycological Society 62: 433-494.

Revell LJ. 2012. phytools: An R package for phylogenetic comparative biology, and other things. Methods in Ecology and Evolution 3: 217-223.

Riley GJP. 1978. The subcellular distribution of carotenoids in Phycomyces blakesleeanus. PhD thesis, Department of Biochemistry, University of London, United Kingdom.

Riley R, Salamov AA, Brown DW, et al. 2014. Extensive sampling of basidiomycete genomes demonstrates inadequacy of the white-rot/brown-rot paradigm for wood decay fungi. Proceedings of the National Academy of Sciences of the United States of America 111 (27): 9923-9928.

Ronquist F, Teslenko M, Mark P, et al. 2012. MrBayes 3.2: Efficient Bayesian phylogenetic inference and model choice across a large model space. Systematic Biology 61: 539-542.

Saccardo PA. 1884. Sylloge Fungorum: Sylloge Sphaeropsidearum et Melanconiearum. Sylloge Fungorum 3: 1-840.

Santamaria M, Fosso B, Licciulli F, et al. 2017. ITSoneDB: a comprehensive collection of eukaryotic ribosomal RNA Internal Transcribed Spacer 1. ITS1 sequences. Nucleic Acids Research 46 (D1): D127-D132. doi: https://doi. org/10.1093/nar/gkx855.

Sayyari E, Mirarab S. 2016. Fast coalescent-based computation of local branch support from quartet frequencies. Molecular Biology and Evolution 33: $1654-1668$

Schumacher T. 1990. The genus Scutellinia. Pyronemataceae. Opera Botanica 101: 1-107.

Seifert KA. 1983. Decay of wood by the Dacrymycetales. Mycologia 75 : 1011-1018.

Shirouzu T, Hirose D, Oberwinkler F, et al. 2013. Combined molecular and morphological data for improving phylogenetic hypotheses in Dacrymycetes. Mycologia 105: 1110-1125.

Shirouzu T, Hirose D, Tokumasu S. 2007. Sequence analyses of $28 \mathrm{~S}$ rRNA gene D1/D2 region suggests Dacrymyces. Heterobasidiomycetes, Dacrymycetales is polyphyletic. Mycoscience 48: 388-394.

Shirouzu T, Hirose D, Tokumasu S. 2009. Taxonomic study of the Japanese Dacrymycetes. Persoonia 23: 16-34.

Shirouzu T, Hosaka K, Nam K-O, et al. 2017. Phylogenetic relationships of eight new Dacrymycetes collected from New Zealand. Persoonia 38: 156169.

Shirouzu T, Uno K, Hosaka K, et al. 2016. Early-diverging wood-decaying fungi detected using three complementary sampling methods. Molecular Phylogenetics and Evolution 98: 11-20.

Simmons MP, Ochoterena H. 2000. Gaps as characters in sequence-based phylogenetic analyses. Systematic Biology 49: 369-381.

Stalpers JA. 1978. Identification of wood-inhabiting Aphyllophorales in pure culture. Studies in Mycology 16: 1-248.

Stielow JB, Lévesque CA, Seifert KA, et al. 2015. One fungus, which genes? Development and assessment of universal primers for potential secondary fungal DNA barcodes. Persoonia 35: 242-263.

Stiller JW, Hall BD. 1997. The origin of red algae: implications for plastid evolution. Proceedings of the National Academy of Sciences of the United States of America 94: 4520-4525

Suchard MA, Redelings BD. 2006. BAli-Phy: simultaneous Bayesian inference of alignment and phylogeny. Bioinformatics 22: 2047-2048.

Sukumaran J, Knowles LL. 2017. Multispecies coalescent delimits structure, not species. Proceedings of the National Academy of Sciences of the United States of America 114: 1607-1612.

The Angiosperm Phylogeny Group [Chase MW, Christenhusz MJM, Fay MF, et al.]. 2016. An update of the Angiosperm Phylogeny Group classification for the orders and families of flowering plants: APG IV. Botanical Journal of the Linnean Society 181: 1-20.
Thiers B. 2019. Index Herbariorum: A global directory of public herbaria and associated staff. New York Botanical Garden's Virtual Herbarium. http:// sweetgum.nybg.org/ih/ [accessed 01 Jan. 2019].

Torkelsen A-E. 1997. Dacryomycetales Lindau. In: Hansen L, Knudsen H (eds), Nordic Macromycetes, volume 3: Heterobasidioid, aphyllophoroid and gastromycetoid Basidiomycetes: 90-96. Nordsvamp, Denmark.

Turland NJ, Wiersema JH, Barrie FR, et al. 2018. International Code of Nomenclature for algae, fungi, and plants. Shenzhen Code adopted by the Nineteenth International Botanical Congress Shenzhen, China, July 2017. Regnum Vegetabile 159: 1-254.

Vail WJ, Lilly VG. 1968. The location of carotenoid pigments and thickness of the cell wall in light- and dark-grown cells of Dacryopinax spathularia. Mycologia 60: 902-907.

Van de Put K. 2014. Basidiemorfologie bij Dacrymyces. Sterbeeckia 33: 41-44.

Vandendries R. 1938 '1937'. Les modalités sexuelles des Basidiomycètes. Bulletin de la Société Royale de Botanique de Belgique 70: 66-85.

Varga T, Krizsán K, Földi C, et al. 2019. Megaphylogeny resolves global patterns of mushroom evolution. Nature Ecology \& Evolution 3: 668-678. doi: https://doi.org/10.1038/s41559-019-0834-1.

Vidal JM, Alvarado P, Loizides M, et al. 2019. A phylogenetic and taxonomic revision of sequestrate Russulaceae in Mediterranean and temperate Europe. Persoonia 42: 127-185.

Vilgalys R, Hester M. 1990. Rapid genetic identification and mapping of enzymatically amplified DNA from several Cryptococcus species. Journal of Bacteriology 172: 4238-4246.

Wang P, Zhang Y, Sha T, et al. 2017. The complete mitochondrial genome of the edible basidiomycete mushroom Thelephora ganbajun. Mitochondrial DNA Part B: Resources 2: 103-105.

Warren DL, Geneva AJ, Lanfear R. 2017. RWTY. R We There Yet?: An R package for examining convergence of Bayesian phylogenetic analyses. Molecular Biology and Evolution 34: 1016-1020.

Wells K. 1994. Jelly fungi then and now! Mycologia 86: 18-48.

White TJ, Bruns T, Lee S, et al. 1990. Amplification and direct sequencing of fungal ribosomal RNA genes for phylogenetics. In: Innis M, Gelfand J, Sninsky J, et al. (eds), PCR protocols: A guide to methods and applications: 315-322. Academic Press, USA.

Will OH, Newland NA, Reppe CR. 1984. Photosensitivity of pigmented and nonpigmented strains of Ustilago violacea. Current Microbiology 10 : 295-302.

Will III OA, Scovel CA. 1989. Photoprotective functions of carotenoids. In: Krinsky NI, Mathews-Roth MM, Taylor RF (eds), Carotenoids. Chemistry and Biology: 229-236. Plenum Press, USA and UK.

Worrall J, Anagnost S, Zabel RA. 1997. Comparison of wood decay among diverse lignicolous fungi. Mycologia 89: 199-219.

Yang Z. 2006. Computational molecular evolution. Oxford, Oxford University Press.

Zamora JC, Millanes AM, Wedin M, et al. 2016. Understanding lichenicolous heterobasidiomycetes: new taxa and reproductive innovations in Tremella s.lat. Mycologia 108: 381-396.

Zamora JC, Pérez-Ortega S, Rico VJ. 2014. Heteroacanthella ellipsospora sp. nov., the first lichenicolous heterobasidiomycete with acanthoid basidia. The Lichenologist 46: 17-23.

Zhang C, Rabiee M, Sayyari E, et al. 2018. ASTRAL-III: Polynomial time species tree reconstruction from partially resolved gene trees. BMC Bioinformatics 19 (S6): 153.

Zhao R-L, Li G-J, Sánchez-Ramírez S, et al. 2017. A six-gene phylogenetic overview of Basidiomycota and allied phyla with estimated divergence times of higher taxa and a phyloproteomics perspective. Fungal Diversity 84: $43-74$ 